\title{
Segmentação de Imagens pela Transformada Imagem-Floresta com Faixa de Restrição Geodésica
}

\author{
Caio de Moraes Braz \\ DisSERTAÇÃO APRESENTADA \\ $\mathrm{AO}$ \\ Instituto de Matemática e Estatística \\ DA \\ Universidade DE SÃO PAUlO \\ PARA \\ OBTENÇÃO DO TÍTULO \\ $\mathrm{DE}$ \\ Mestre em CiênCia da ComputaÇão
}

Programa: Mestrado em Ciência da Computação

Orientador: Prof. Dr. Paulo André Vechiatto de

Miranda - IME-USP

Durante o desenvolvimento deste trabalho o autor recebeu auxílio financeiro da CAPES 


\section{Segmentação de Imagens pela Transformada Imagem-Floresta com Faixa de Restrição Geodésica}

\footnotetext{
Esta versão da dissertação contém as correções e alterações sugeridas pela Comissão Julgadora durante a defesa da versão original do trabalho, realizada em 24/02/2016. Uma cópia da versão original está disponível no Instituto de Matemática e Estatística da Universidade de São Paulo.
}

Comissão Julgadora:

- Prof. Dr. Paulo André Vechiatto de Miranda - IME-USP

- Prof. Dr. Marcel Parolin Jackowski - IME-USP

- Prof $^{\mathrm{a}}$. Dra. Leticia Rittner - UNICAMP 


\section{Agradecimentos}

Agradeço ao meu orientador Prof. Paulo Miranda por todo apoio e ajuda durante este projeto; à minha família pelo suporte fornecido e pela compreensão nos momentos de ausência deste período; aos amigos de longa data tanto do IME quanto de fora, que sempre estiveram, estão e estarão disponíveis para conversar sobre todo tipo de assunto e principalmente serem as pessoas em qual eu posso confiar pra tudo; aos colegas do laboratório e-science que foram importantes com seus conselhos e conhecimento; a todos os grandes docentes com os quais tive a honra de aprender algo; ao Instituto de Matemática e Estatística pela sua estrutura que permitiu que este trabalho fosse em frente, incluindo a estrutura do laboratório e-science (FAPESP grant \# 2011/50761-2, CNPq, CAPES, NAP eScience - PRP - USP), e à CAPES pelo apoio financeiro que possibilitou a dedicação exclusiva a este trabalho. 


\section{Resumo}

BRAZ, C. Segmentação de Imagens pela Transformada Imagem-Floresta com Faixa de Restrição Geodésica. 2015. 33 f. Dissertação (Mestrado) - Instituto de Matemática e Estatística, Universidade de São Paulo, São Paulo, 2015.

Vários métodos tradicionais de segmentação de imagens, como a transformada de watershed de marcadores e métodos de conexidade fuzzy (Relative Fuzzy Connectedness- RFC, Iterative Relative Fuzzy Connectedness - IRFC), podem ser implementados de modo eficiente utilizando o método em grafos da Transformada Imagem-Floresta (Image Foresting Transform - IFT). No entanto, a carência de termos de regularização de fronteira em sua formulação fazem com que a borda do objeto segmentado possa ser altamente irregular. Um modo de contornar isto é por meio do uso de restrições de forma do objeto, que favoreçam formas mais regulares, como na recente restrição de convexidade geodésica em estrela (Geodesic Star Convexity - GSC).

Neste trabalho, apresentamos uma nova restrição de forma, chamada de Faixa de Restrição Geodésica (Geodesic Band Constraint - GBC), que pode ser incorporada eficientemente em uma sub-classe do framework de corte em grafos generalizado (Generalized Graph Cut - GGC), que inclui métodos pela IFT. É apresentada uma prova da otimalidade do novo algoritmo em termos de um mínimo global de uma função de energia sujeita às novas restrições de borda. A faixa de restrição geodésica nos ajuda a regularizar a borda dos objetos, consequentemente melhorando a segmentação de objetos com formas mais regulares, mantendo o baixo custo computacional da IFT. A GBC pode também ser usada conjuntamente com um mapa de custos pré estabelecido, baseado em um modelo de forma, de modo a direcionar a segmentação a seguir uma dada forma desejada, com grau de liberdade de escala e demais deformações controladas por um parâmetro único. Essa nova restrição também pode ser combinada com a GSC e com as restrições de polaridade de borda sem custo adicional. O método é demonstrado em imagens naturais, sintéticas e médicas, sendo estas provenientes de tomografias computadorizadas e de ressonância magnética.

Palavras-chave: faixa de restrição geodésica, convexidade geodésica em estrela, transformada imagem floresta, segmentação por corte em grafos, conexidade fuzzy. 


\section{Sumário}

Lista de Figuras $\quad$ vii

1 Introdução $\quad 1$

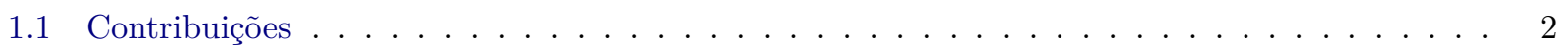

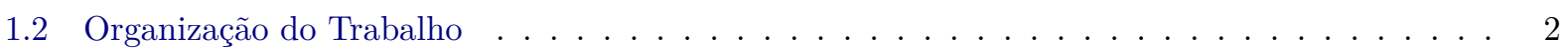

2 Transformada Imagem-Floresta $\quad \mathbf{5}$

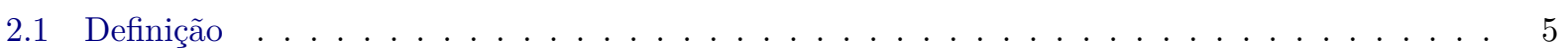

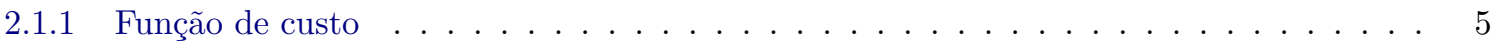

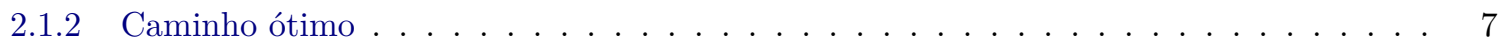

2.1 .3 Função de custo suave $\ldots \ldots \ldots \ldots \ldots \ldots \ldots \ldots$

2.2 Segmentação por competição de sementes $\ldots \ldots \ldots \ldots \ldots \ldots \ldots \ldots$

2.2 .1 Algoritmo geral da IFT $\ldots \ldots \ldots \ldots \ldots \ldots$

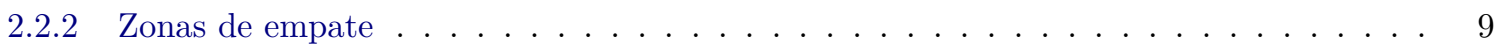

3 Corte em Grafos Generalizado $\quad 11$

3.1 Definição do framework . . . . . . . . . . . . . . . . . . . . . . . . 11

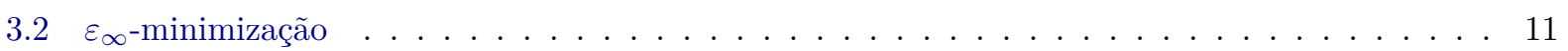

4 Convexidade Geodésica em Estrela $\quad 13$

4.1 Convexidade em estrela . . . . . . . . . . . . . . . . . . . . . . . . . . . 13

4.2 Segmentação com restrições de convexidade geodésica em estrela . . . . . . . . . . . . . . . 14

5 Transformada Imagem-Floresta com Orientação de Bordas - OIFT 17

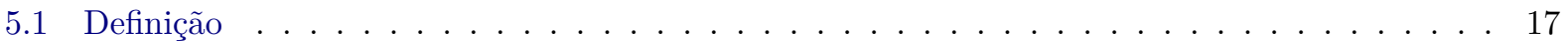

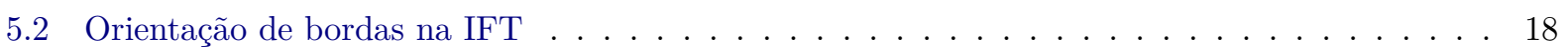

6 Faixa de Restrição Geodésica $\quad 21$

6.1 Faixa de Restrição . . . . . . . . . . . . . . . . . . . . . . . . . . . . . . 21

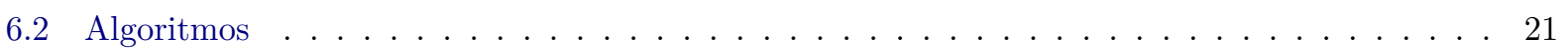

6.2 .1 Corretude do Algoritmo GBC-IFT . . . . . . . . . . . . . . . . . 24

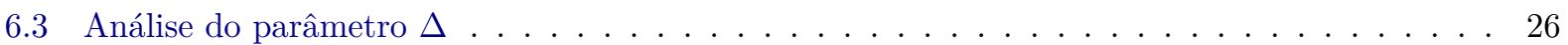

6.4 Combinação com outras restrições $\ldots \ldots \ldots \ldots \ldots \ldots \ldots \ldots \ldots$

7 Faixa de Restrição Geodésica Relativa $\quad 31$

7.1 Faixa de Restrição Relativa . . . . . . . . . . . . . . . . . . . . . . . . . . . . . . 31

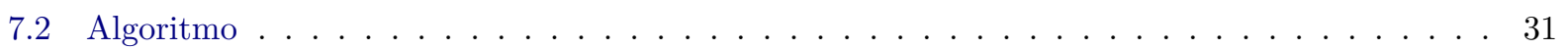

7.3 Modelos pré-definidos de mapas de custos $\ldots \ldots \ldots \ldots \ldots \ldots \ldots \ldots$

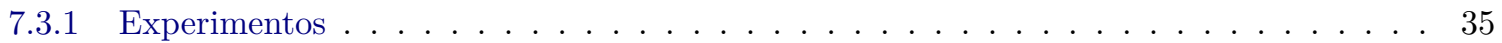


8 Conclusão e trabalhos futuros

8.1 Considerações Finais . . . . . . . . . . . . . . . . . . . . . . . . . . . . 41

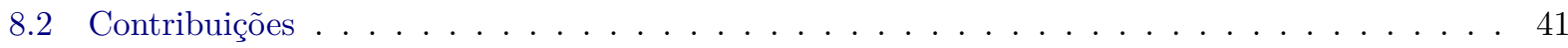

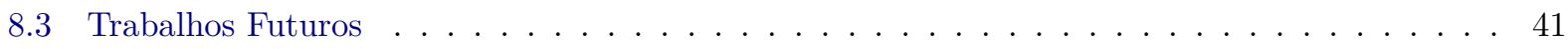

Referências Bibliográficas $\quad 43$ 


\section{Lista de Figuras}

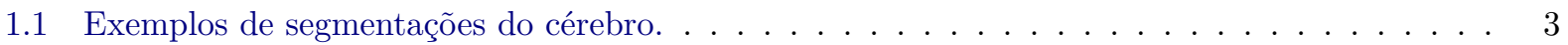

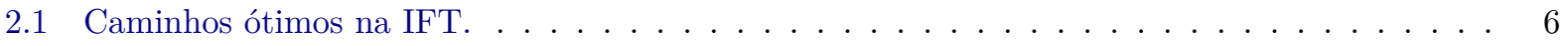

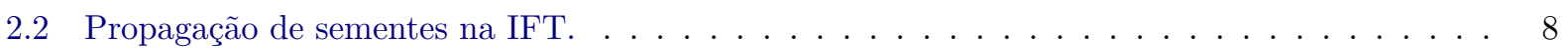

2.3 Exemplo de execução da IFT. . . . . . . . . . . . . . . . . . . . . . . . . . 9

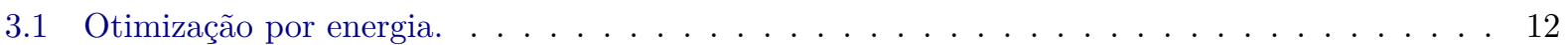

4.1 Definição de convexidade em forma de estrela. . . . . . . . . . . . . . . . . . . . . . 13

4.2 Visibilidade do centro na GSC . . . . . . . . . . . . . . . . . . . . . . 14

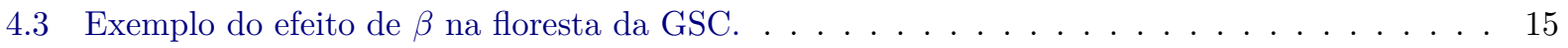

4.4 Exemplo de segmentação e da floresta de caminhos na GSC . . . . . . . . . . . . . . . . 16

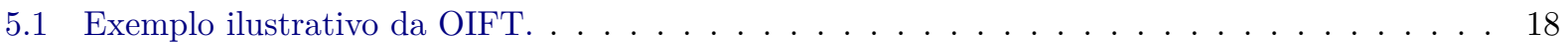

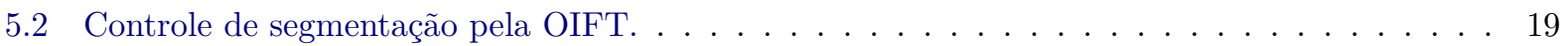

6.1 Evolução da GBC. . . . . . . . . . . . . . . . . . . . . . . . . . . . . 22

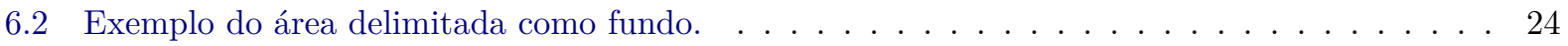

6.3 Comparação entre segmentações com a GBC-IFT (1) . . . . . . . . . . . . . . . . . 27

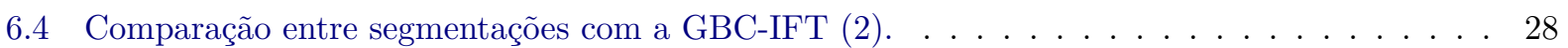

6.5 Comparação entre outros métodos e a GBC-IFT (1) . . . . . . . . . . . . . . . . . . 29

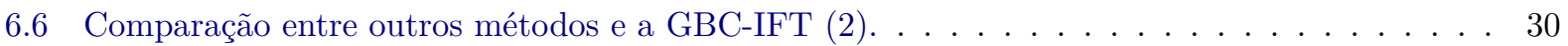

7.1 Limitações da GBC-IFT. . . . . . . . . . . . . . . . . . . . . . . 32

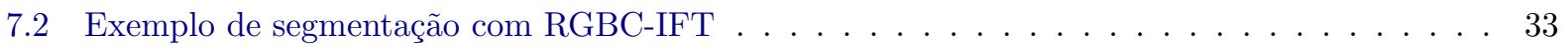

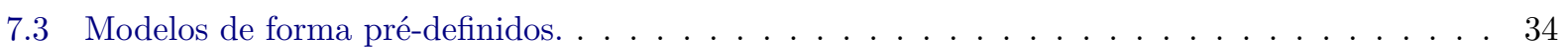

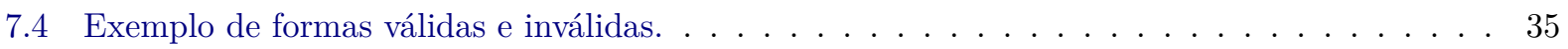

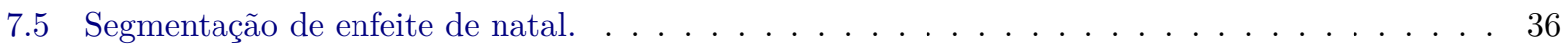

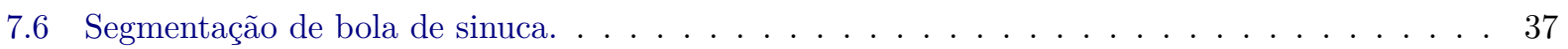

7.7 Segmentação de azulejo $(1) \ldots \ldots \ldots \ldots \ldots \ldots$

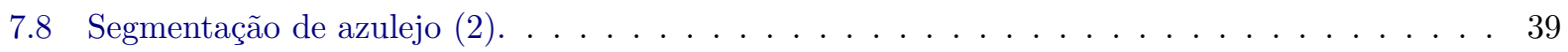

7.9 Segmentação de azulejo $(3) \ldots \ldots \ldots \ldots \ldots$ 


\section{Capítulo 1}

\section{Introdução}

A segmentação de imagens, que consiste em particioná-la em regiões relevantes para uma dada aplicação, é muito útil em vários domínios como análise de imagens médicas e biológicas, edição e composição de imagens naturais, reconhecimento de caracteres, entre outros [GW92]. Ela é um dos problemas fundamentais e mais desafiadores na área de processamento de imagens e visão computacional. No âmbito de imagens médicas, o conhecimento de alto nível do especialista, específico ao domínio da aplicação, geralmente é necessário para a segmentação devido a presença de estruturas mal definidas, falta de padrão de intensidade entre imagens, inomogeneidade de campo magnético em imagens de ressonância magnética, ruídos, efeitos de volume parcial e sua ação combinada [OS01]. Em publicidade, a edição de imagens e vídeos muitas vezes necessita de uma segmentação guiada pelo usuário devido à sua natureza heterogênea [BS07, WC05]. Este processo de segmentação guiada pelo usuário é chamado de segmentação interativa.

Dada a natureza discreta das imagens digitais, a matemática discreta baseada em grafos emergiu como uma ferramenta perfeita e unificadora para representar, processar e analisar imagens [LG12]. Como consequência, essa área tem despertado grande interesse nos últimos anos, conforme atestam a crescente quantidade de artigos publicados na área [CG12], as apresentações inteiramente dedicadas ao tema por palestrantes de renome internacional em congressos e convenções e até livros exclusivamente dedicados ao tópico [LG12].

A interpretação de uma imagem digital como um grafo pode ocorrer em diferentes níveis, podendo os vértices do grafo representarem diferentes elementos da imagem tais como pixels, regiões de pixels ou até objetos da imagem com valor semântico agregado. Até mesmo um banco de dados de imagens pode ser interpretado como um grafo cujos vértices são as imagens. Em qualquer caso, cada vértice possui um conjunto associado de atributos da imagem e as arestas descrevem relações binárias entre eles. Neste cenário, os operadores de imagem (por exemplo: filtragem, segmentação, representação e descrição de objetos e classificação) podem tirar proveito de uma literatura rica e dinâmica.

Uma classe importante da segmentação interativa de imagens diz respeito aos métodos baseados em sementes [CG12], que vem sendo desenvolvidos baseados em diferentes teorias, supostamente não relacionadas, levando a diferentes frameworks como o watershed [CBNC10], passeios aleátorios [Gra06], conexidade fuzzy [CUSZ07], corte em grafos [BJ01], problema do caminho mínimo [BS07, VK05] e transformada imagemfloresta (IFT) [FSL04]. O estudo das relações entre os diferentes frameworks possui uma vasta bibliografia $\left[\mathrm{AAC}^{+}\right.$07, SL07, CUFM12a, CLLH10], o que permitiu com que muitos algoritmos fossem descritos de uma maneira unificada de acordo com um framework comum, chamado de Corte em Grafo Generalizado (Generalized Graph Cut - GGC) [CUFM12b, CLLH10]. Nesses métodos, o usuário fornece uma rotulação parcial da imagem, selecionando alguns de seus pixels como sementes. Estes pixels propagam os seus rótulos para os demais pixels de modo a minimizar uma energia para uma partição ótima da imagem. Correções na segmentação obtida podem ser feitas por meio da adição ou remoção de sementes, que restringem o universo de possíveis rotulações [FB04].

Dentro deste framework duas classes importantes de formulação de energia se destacam, os problemas da $\varepsilon_{1}$ e da $\varepsilon_{\infty}$-minimização (e os seus algoritmos), como estudado em [CUFM12b]. Apesar do sucesso conseguido em várias aplicações e a popularidade de abordagens relacionadas ao problema da $\varepsilon_{\infty}$-minimização, como a watershed de marcadores [CBNC10, MF09, AL07], iterative relative fuzzy connectedness [CUFM12a] e a transformada imagem-floresta [MF09], a falta de restrições de regularidade, em geral faz com que estes métodos sejam inapropriados (ou pelo menos não a melhor escolha) para alguns domínios de aplicação. Por exemplo, quando a forma esperada do objeto é simples e regular, a presença de segmentos falhos na borda pode gerar problemas de falsos positivos (vazamentos) nas segmentações pelos métodos citados anteriormente, resultando não apenas em segmentações erradas mas também com bordas irregulares (Figura 1.1a). Por outro lado, os métodos baseados no problema da $\varepsilon_{1}$-minimização (e.g. fluxo máximo-corte mínimo [BK04]) têm uma componente regularizadora intrínseca em suas formulações, sendo menos suscetíveis aos vazamentos 
descritos acima, no entanto isso geralmente vem acompanhado de certas contrapartidas, como o problema do encolhimento, onde há uma tendência aos objetos segmentados terem tamanhos menores do que o real, e também a custos computacionais mais elevados, como por exemplo, ordem de complexidade $N^{2.5}$, sendo $N$ a quantidade de pixels da imagem [LG12]. Os métodos de $\varepsilon_{1}$ e $\varepsilon_{\infty}$-minimização podem também ser estendidos para grafos dirigidos, permitindo a incorporação da polaridade de borda, de modo a favorecer a segmentação de objetos com transições predominantemente escuro para claro ou vice-versa (Figura 1.1c) [MM14].

\subsection{Contribuições}

Este trabalho recai dentro do problema da $\varepsilon_{\infty}$-minimização do framework GGC e é motivado por alguns avanços recentes, como a introdução da restrição de convexidade geodésica em estrela (Geodesic Star Convexity - GSC) de Gulshan et al. [GRC ${ }^{+}$10] na transformada imagem floresta [MJM13]. Esta restrição de borda no problema da $\varepsilon_{\infty}$-minimização elimina formas irregulares indesejadas, melhorando a segmentação de objetos com uma forma mais regular (Figura 1.1b), mantendo o baixo custo computacional da IFT (implementações em tempo linear [FSL04]). Partindo da mesma ideia, apresentamos uma nova restrição de borda, que chamamos de Faixa de Restrição Geodésica (Geodesic Band Constraint - GBC) e mostramos um novo algoritmo para uma implementação eficiente da IFT sujeita a esta restrição, que chamaremos de GBC-IFT.

O novo algoritmo calcula uma solução ótima global para o problema da $\varepsilon_{\infty}$-minimização sujeita às novas restrições (Figura 1.1d). Esta restrição também pode ser combinada com a restrição GSC e com as restrições de polaridade de borda [MM14, MM13b], permitindo uma melhor customização do problema da $\varepsilon_{\infty}$-minimização para um objeto alvo de interesse [LG12] e pode também ser usada conjuntamente com um mapa de custos pré-definido, que representa um modelo de formas em diversas escalas, favorecendo a obtenção de uma segmentação com forma semelhante à forma desejada, sendo as deformações controladas por um único parâmetro, que pode ser absoluto ou relativo, gerando assim dois métodos distintos.

\subsection{Organização do Trabalho}

Primeiramente introduzimos alguns fundamentos sobre imagens digitais e sobre a Transformada ImagemFloresta no Capítulo 2, seguido da definição do framework de Corte em Grafos Generalizado no Capítulo 3, que será nossa base teórica.

Após isso, veremos a restrição de polaridade de bordas (OIFT) no Capítulo 5, na qual podemos usar a orientação das arestas grafo da imagem a nosso favor. No Capítulo 4 veremos a restrição de Convexidade Geodésica em Estrela, que é uma restrição de forma que ataca o problema da regularização de uma forma distinta da nossa nova restrição que apresentamos no Capítulo 6, onde mostramos nosso trabalho original, a Faixa de Restrição Geodésica, um novo algoritmo cuja ideia é regularizar a forma das segmentações limitando uma faixa onde a borda da segmentação deve estar

No Capítulo 7, apresentamos uma evolução deste algoritmo, onde alteramos o parâmetro da faixa de restrição para um valor relativo, de modo a contornar alguns problemas existentes no método original e analisamos o que é possível ser feito.

Finalmente, apresentamos nossas conclusões e sugestões de trabalhos futuros no Capítulo 8. 


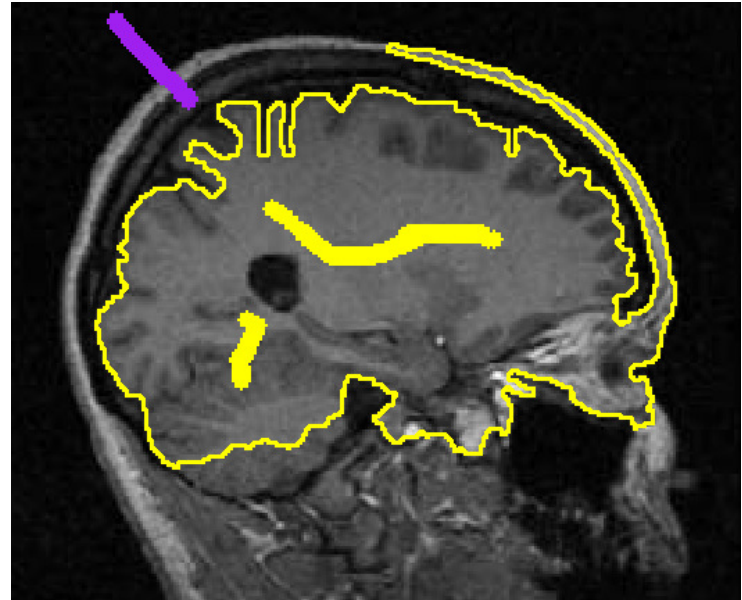

(a) IFT

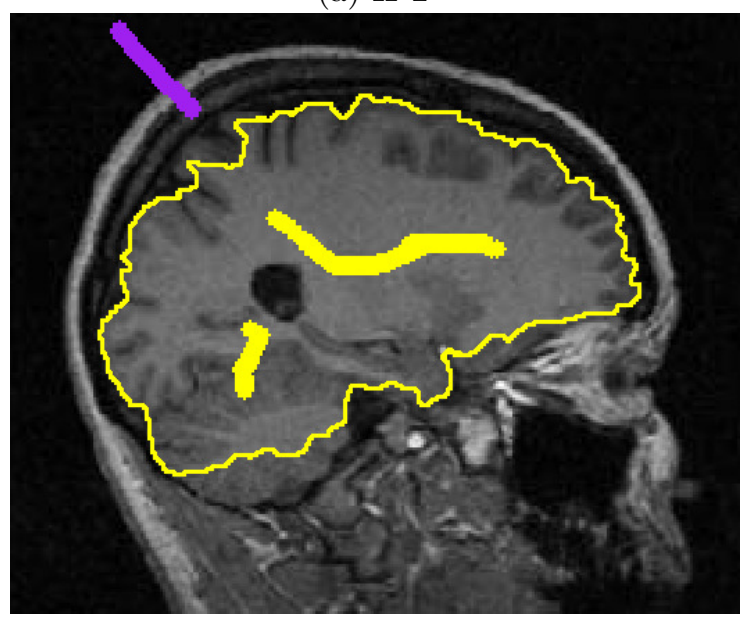

(c) GSC-OIFT

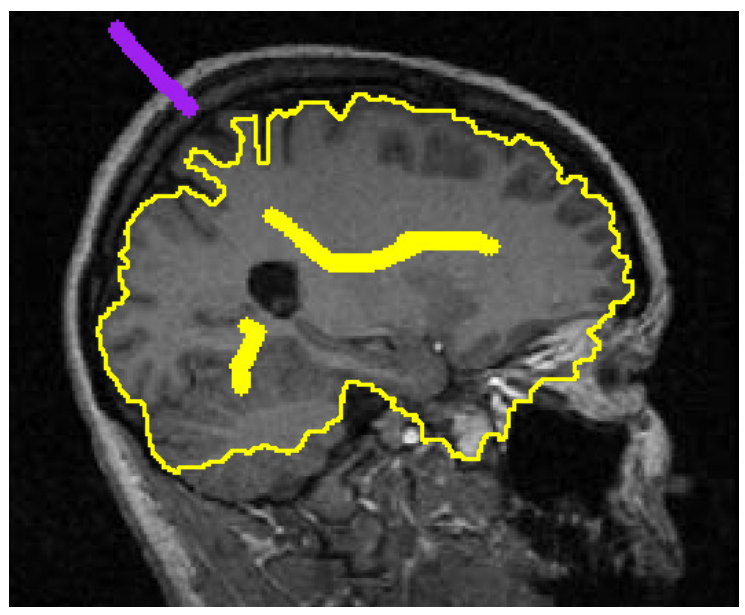

(b) GSC-IFT

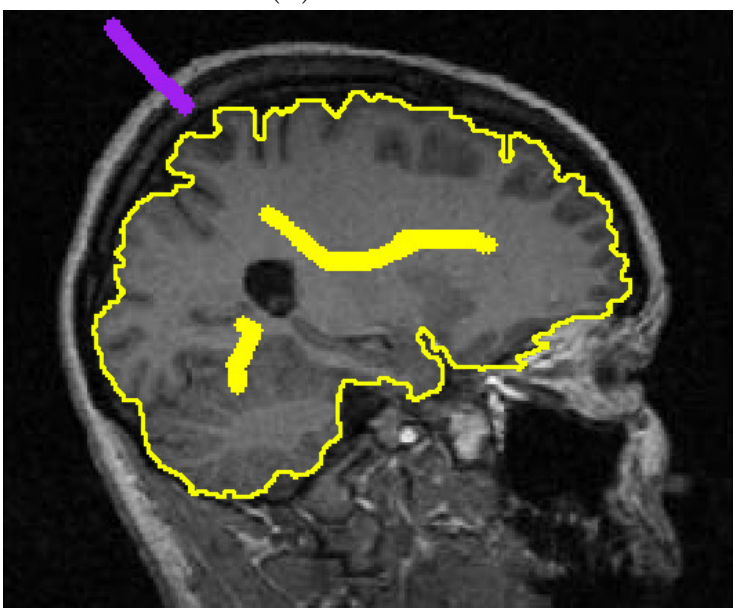

(d) GBC-IFT

Figura 1.1: Exemplos de segmentações do cérebro (skull stripping), realizadas por diferentes algoritmos, para o mesmo conjunto de sementes. 


\section{Capítulo 2}

\section{Transformada Imagem-Floresta}

Neste trabalho é apresetada uma restrição de borda para ser usada no problema da $\varepsilon_{\infty}$-minimização da segmentação pela Transformada Imagem-Floresta (Image Foresting Transform - IFT), que é uma ferramenta baseada em grafos, para modelar, implementar e avaliar operadores de processamento de imagens baseados em conexidade.

A IFT unifica e estende várias técnicas de processamento de imagens que, mesmo baseadas em conceitos similares, muitas vezes são apresentadas como métodos não relacionados. Ela consiste em reduzir os problemas, como por exemplo, filtragem [FdCL01], segmentação [MFS11, FB04], representação de formas [FdC01], ao cálculo de uma floresta de caminhos ótimos, baseando-se em uma função de custo no grafo derivado da imagem, seguido de um processamento adequado de seus atributos.

Neste capítulo, apresentamos a IFT de modo mais específico para o problema de segmentação baseada em regiões.

\subsection{Definição}

Na IFT uma imagem pode ser vista como um grafo dirigido $G=(\mathcal{I}, \mathcal{A})$ onde os vértices são os pixels da imagem no seu domínio $\mathcal{I} \subset \mathbb{Z}^{n}$ e os arcos são o pares ordenados de pixels $(s, t) \in \mathcal{A}$ definidos por uma relação de adjacência $\mathcal{A}$ (por exemplo, vizinhança-4, vizinhança-8, etc...). Cada arco $(s, t) \in \mathcal{A}$ tem um peso $\omega(s, t) \geq 0$ associado, que pode ser dado por uma medida de dissimilaridade entre os pixels $s$ e $t$ (por exemplo, $\omega(s, t)=|I(t)-I(s)|$ para uma imagem com um só canal, com valores dados por $I(t)$ ). Um caminho $\pi_{t}=\left\langle t_{1}, t_{2}, \ldots, t_{n}=t\right\rangle$ nesse grafo é uma sequência de pixels adjacentes com término no pixel $t$. Todos os caminhos considerados nesse trabalho são caminhos simples, isto é, passam por cada vértice no máximo uma vez. Usamos a notação $\pi_{t}=\langle t\rangle$ para indicar um caminho composto por um único vértice, o qual denotamos por caminho trivial. A notação $\pi_{t} \cdot\left\langle s_{1}=t, s_{2}, \ldots, s_{m}\right\rangle$ é usada para indicar a extensão de um caminho $\pi_{t}$ por outro que tenha início em seu vértice terminal $t$. Por exemplo, a expressão $\pi_{t}=\pi_{s} \cdot\langle s, t\rangle$ é usada para indicar a extensão de um caminho $\pi_{s}$ com término em $s$, por um arco $(s, t) \in \mathcal{A}$ (Figura 2.1(a)). Quando queremos indicar explicitamente a origem de um caminho, a notação $\pi_{r \rightsquigarrow t}=\left\langle s_{1}=r, s_{2}, \ldots, s_{m}=t\right\rangle$ também pode ser usada, com $r$ correspondendo à origem e $t$ o nó destino. Cada caminho $\pi_{t}$ possui um valor de conexidade $f\left(\pi_{t}\right)$ associado, definido por uma função $f$ de custo do caminho, que normalmente depende de propriedades locais da imagem, tais como cor, gradiente e posição do vértice ao longo do caminho.

No framework da IFT consideramos um mapa de predecessores que é uma função $P$ que associa a cada pixel $t \in \mathcal{I}$ algum outro pixel adjacente em $\mathcal{I}$, ou um marcador distinto nil $\notin \mathcal{I}$. Nesse último caso $t$ é uma raiz do mapa (Figura 2.1(b)). Uma floresta de espalhamento é um mapa $P$ de predecessores que não contém ciclos, isto é, voltando pelo mapa $P$ a partir de qualquer vértice, chegamos a nil em um número finito de iterações (Figura 2.1(c)). Assim, para qualquer pixel $t \in \mathcal{I}$, a floresta $P$ define um caminho $\pi_{t}^{P}$ recursivamente como $\langle t\rangle$, se $P(t)=$ nil e $\pi_{s}^{P} \cdot\langle s, t\rangle$ se $P(t)=s \neq n i l$.

\subsubsection{Função de custo}

Uma função de custo $f$ associa a um caminho $\pi_{t}$ no grafo $G=(\mathcal{I}, \mathcal{A})$ um valor de custo $f\left(\pi_{t}\right)$ em algum conjunto totalmente ordenado $F$ de valores de custo, sendo que o seu inverso indica a força de conexidade entre o pixel $t$ e o nó raiz do caminho $R\left(\pi_{t}\right)$. Em geral, $f$ é baseada nos pesos das arestas que compõem o caminho.

Exemplos básicos de funções de custo são as funções $f_{\text {sum }}$ e $f_{\max }$, que são definidas pelas Eq. 2.1.1 e 2.1.2 respectivamente. Na notação temos $(s, t) \in \mathcal{A}, \pi_{s}$ sendo um caminho terminado em $s, H(t)$ é um valor inicial 


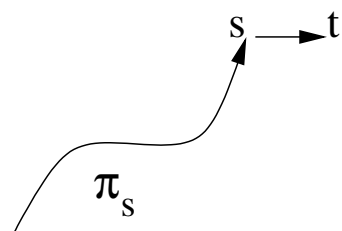

(a)

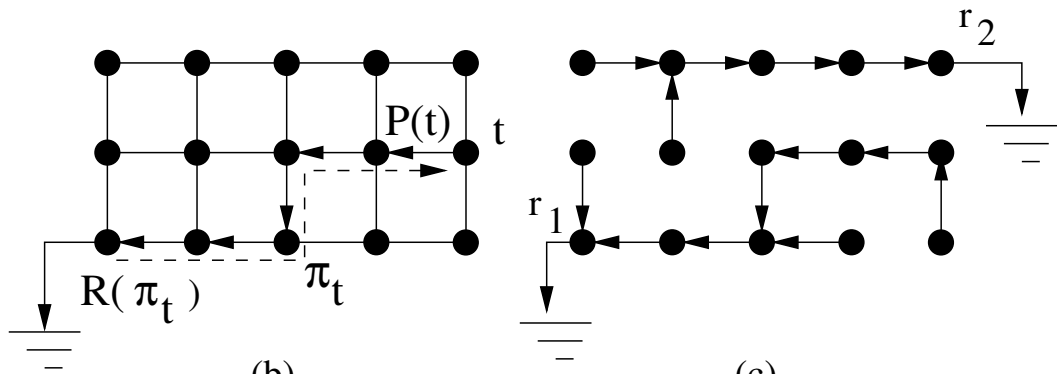

(b)

(c)

Figura 2.1: (a) Extensão de um caminho $\pi_{s}$ pelo arco $(s, t)$. (b) Grafo de vizinhança-4 mostra um caminho $\pi_{t}$ (linha pontilhada) representado no sentido inverso onde $P(t)$ é o nó predecessor de $t$ e $R\left(\pi_{t}\right)$ é o pixel raiz/origem do caminho $\pi_{t}$. (c) Floresta de espalhamento $P$ com dois nós raizes, $r_{1}$ e $r_{2}$.

(handicap) fixo para um caminho iniciando em $t$ e $\delta(s, t)$ é um peso não negativo associado ao arco $(s, t)$.

$$
\begin{aligned}
f_{\text {sum }}(\langle t\rangle) & =H(t) \\
f_{\text {sum }}\left(\pi_{s} \cdot\langle s, t\rangle\right) & =f_{\text {sum }}\left(\pi_{s}\right)+\delta(s, t), \quad \mathrm{e} \\
f_{\max }(\langle t\rangle) & =H(t) \\
f_{\max }\left(\pi_{s} \cdot\langle s, t\rangle\right) & =\max \left\{f_{\max }\left(\pi_{s}\right), \omega(s, t)\right\}
\end{aligned}
$$

Em geral, o valor inicial $H(t)$ é dado pela Eq. 2.1.3, onde $\mathcal{S} \subseteq \mathcal{I}$ é o conjunto de pixels sementes.

$$
H(t)= \begin{cases}0 & , \text { se } t \in \mathcal{S} \\ +\infty & , \text { caso contrário }\end{cases}
$$

As funções $f_{\text {sum }}$ e $f_{\max }$, entre outras, pertencem a uma classe de funções chamada monotonicamente incrementais $(\mathrm{MI})$, que satisfazem as seguintes expressões:

$$
\begin{aligned}
f_{m i}(\langle t\rangle) & =H(t) \\
f_{m i}\left(\pi_{s} \cdot\langle s, t\rangle\right) & =f_{m i}\left(\pi_{s}\right) \odot(s, t),
\end{aligned}
$$

sendo $H(t)$ uma função arbitrária e o operador binário $\odot: F \times \mathcal{A} \rightarrow F$ que satisfaz as seguintes condições:

(M1) $x^{\prime} \geq x \Rightarrow x^{\prime} \odot(s, t) \geq x \odot(s, t)$,

(M2) $x \odot(s, t) \geq x$

para $x, x^{\prime} \in F$ e qualquer arco $(s, t) \in \mathcal{A}$. Uma característica essencial desta classe de funções é que $\odot$ depende apenas do valor do caminho $\pi_{s}$ e não de outra propriedade dele.

As funções MI podem ser usadas em operações de filtragem, segmentação de imagem, representação e descrição de objetos e classificação de padrões usando caminhos ótimos no grafo $G$.

No entanto, alguns operadores necessitam de funções mais gerais do que as funções MI. Este é o caso da função $f_{\text {euc }}$ usada em problemas que envolvem a transformada de distância Euclideana, definida como:

$$
\begin{aligned}
f_{\text {euc }}(\langle t\rangle) & = \begin{cases}0 & , \text { se } t \in \mathcal{S} \\
+\infty & , \text { caso contrário }\end{cases} \\
f_{\text {euc }}\left(\pi_{s} \cdot\langle s, t\rangle\right) & =\left\|t-R\left(\pi_{s}\right)\right\|^{2}
\end{aligned}
$$

onde $(s, t) \in \mathcal{A}, \pi_{s}$ é um caminho terminado em $s, R\left(\pi_{s}\right)$ é a raiz do caminho $\pi_{s}$, e $\mathcal{S} \subseteq \mathcal{I}$ é um conjunto de sementes. 


\subsubsection{Caminho ótimo}

Seja $\Pi(\mathcal{I}, \mathcal{A}, t)$ o conjunto de todos os caminhos possíveis no grafo $G=(\mathcal{I}, \mathcal{A})$ que terminam em $t$. Um caminho $\pi_{t}$ é ótimo se $f\left(\pi_{t}\right) \leq f\left(\pi_{t}^{\prime}\right)$ para qualquer outro caminho $\pi_{t}^{\prime} \in \Pi(\mathcal{I}, \mathcal{A}, t)$, ou seja, qualquer outro caminho que termine em $t$, independente da sua origem.

O valor $V(t)$ de um caminho ótimo para cada pixel $t \in \mathcal{I}$ é definido como:

$$
V(t)=\min _{\pi_{t} \in \Pi(\mathcal{I}, \mathcal{A}, t)}\left\{f\left(\pi_{t}\right)\right\}
$$

Um caminho $\pi_{t_{n}}=\left\langle t_{1}, t_{2}, \ldots, t_{n}\right\rangle$ é ótimo completo se todos os caminhos $\pi_{t_{i}}=\left\langle t_{1}, t_{2}, \ldots, t_{i}\right\rangle$, para $i=$ $1,2, \ldots, n$, são caminhos ótimos.

Note que qualquer caminho $\pi_{t}^{P}$ definido por uma floresta de caminhos ótimos $P$ é um caminho ótimo completo.

\subsubsection{Função de custo suave}

A IFT para um grafo $G=(\mathcal{I}, \mathcal{A})$ e para uma função de custo suave $f$, associa um caminho ótimo para cada pixel $t \in \mathcal{I}$, obtendo uma floresta $P$ de caminhos ótimos, isto é, uma floresta $P$ de espalhamento onde todos os caminhos $\pi_{t}^{P}$ são ótimos. Não há garantias de que os caminhos gerados sejam ótimos caso a função $f$ não seja suave [FSL04].

Uma função de custo $f$ é suave se para todo vértice $t \in \mathcal{I}$, existe um caminho ótimo $\pi_{t}$, de modo que para todo $\tau$ e $\delta$ tais que $\pi_{t}=\tau \cdot \delta$, temos:

(C1) $f(\tau) \leq f\left(\pi_{t}\right)$.

(C2) $\tau$ é ótimo.

(C3) Para qualquer caminho ótimo $\tau^{\prime}$ com o mesmo vértice terminal de $\tau, f\left(\tau^{\prime} \cdot \delta\right)=f\left(\pi_{t}\right)$.

Observe que a classe das funções suaves é menos restrita que a classe de funções MI.

\subsection{Segmentação por competição de sementes}

Na segmentação via IFT por competição de sementes (IFT Segmentation by Seed Competition - IFT$\mathrm{SC}$ ), consideramos dois conjuntos de sementes, um conjunto $\mathcal{S}_{o}$ de sementes internas, composto por pixels selecionados dentro do objeto e um conjunto $\mathcal{S}_{b}$ de sementes externas composto por pixels selecionados dentro do fundo (background), onde $\mathcal{S}_{o} \cap \mathcal{S}_{b}=\emptyset$. Neste paradigma, as sementes $\mathcal{S}_{o}$ e $\mathcal{S}_{b}$ competem tentando "conquistar" os demais pixels da imagem (Figura 2.2), de modo que é criada uma partição em duas florestas de caminhos ótimos, uma enraizada nas sementes internas, definindo o objeto, e outra enraizada nas sementes externas, representando o fundo [MF09].

O resultado da segmentação é dado pela imagem rotulada $L$, definida por:

$$
L(t)= \begin{cases}1 & \text { se } R\left(\pi_{t}^{P}\right) \in \mathcal{S}_{o} \\ 0 & \text { caso contrário. }\end{cases}
$$

onde $\pi_{t}^{P}$ é um caminho ótimo que termina no pixel $t$, obtido a partir de $P$.

Para uma implementação mais eficiente, o Algoritmo 1 propaga os rótulos das raízes, isto é, as sementes, para todos os vértices do grafo $G$ durante a sua execução. 

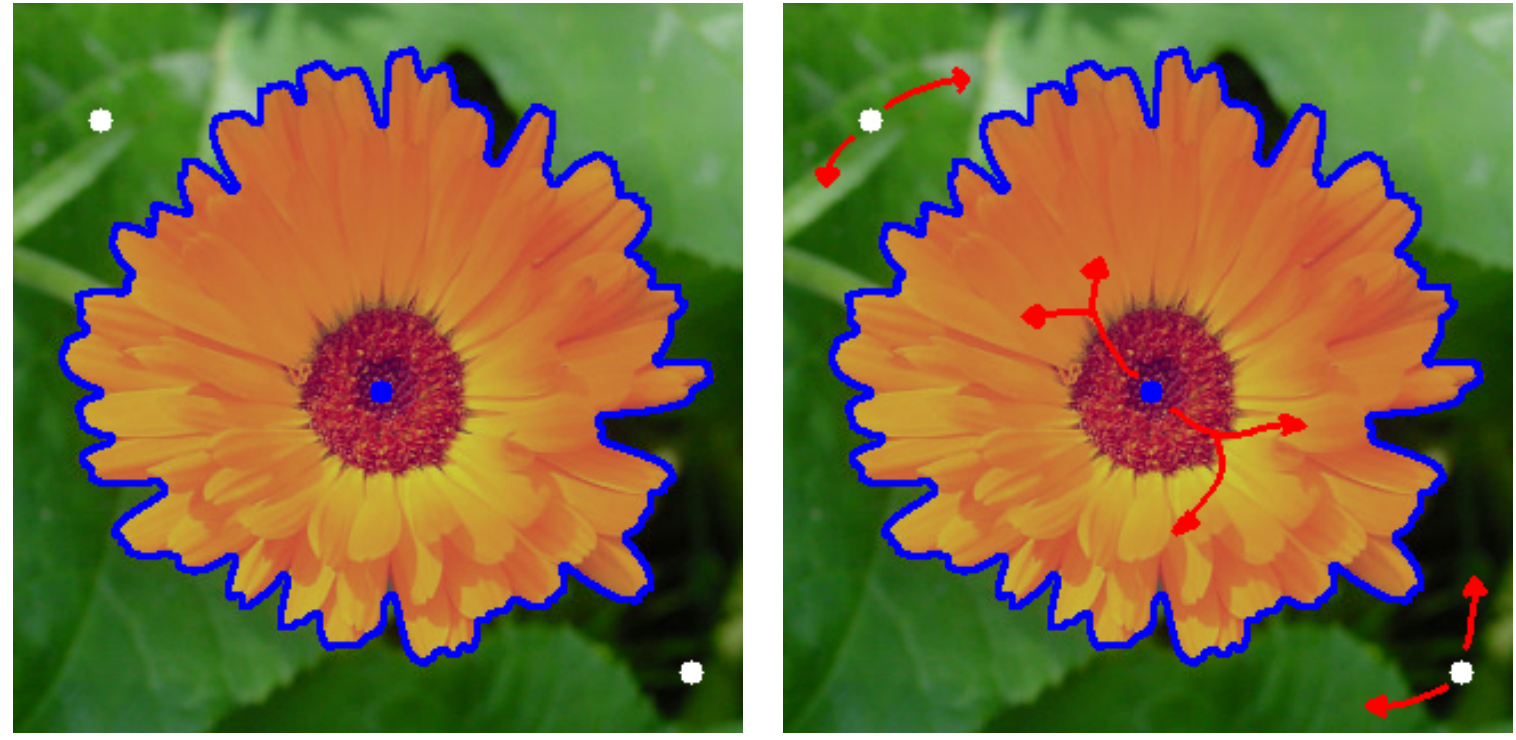

Figura 2.2: Propagação das sementes após execução da IFT. Em branco as sementes de fundo e em azul as sementes de objeto.

\subsubsection{Algoritmo geral da IFT}

O algoritmo da IFT é essencialmente o algoritmo de Dijkstra para o cálculo de caminhos de valor mínimo [Dij59, AMO93], ligeiramente modificado para permitir fontes múltiplas e função mais geral de custo, isto é, obtém uma floresta de caminhos ótimos $P$ minimizando uma função de custo suave $f$.

\section{Algoritmo 1. Algoritmo geral IFT}

Entrada: $\quad$ Grafo dirigido $G=(\mathcal{I}, \mathcal{A})$, adjacência $A$, conjuntos $\mathcal{S}_{o}$ e $\mathcal{S}_{b}$ de sementes e função de custo suave $f$. SAÍdA: $\quad$ Floresta $P$ de caminhos ótimos, mapa $V$ de valores mínimos e mapa $L$ de rótulos.

Auxiliares: Fila $Q$ de prioridade, variável tmp, e um array estado de vértices.

1. Para cada $t \in \mathcal{S}_{o}$, Faça $L(t) \leftarrow 1$

2. Para cada $t \in \mathcal{S}_{b}$, Faça $L(t) \leftarrow 0$

3. Para cada $t \in \mathcal{I}$, Faça

4. $P(t) \leftarrow$ nil $e V(t) \leftarrow f(\langle t\rangle)$.

5. $\quad \operatorname{estado}(t) \leftarrow 0$.

6. Se $V(t) \neq+\infty$, então insira $t$ em $Q$.

7. Enquanto $Q \neq \emptyset$, Faça

8. Remova s de $Q$ cujo valor $V(s)$ é mínimo.

9. $\quad \operatorname{estado}(s) \leftarrow 1$.

10. Para cada $t \in \mathcal{A}(s)$, tal que estado $(t)=0$, Faça

11. Calcule tmp $\leftarrow f\left(\pi_{s}^{P} \cdot\langle s, t\rangle\right)$.

12. Se $\operatorname{tmp}<V(t)$, então

13. Se $V(t) \neq+\infty$, então remova $t$ de $Q$.

14. Faça $P(t) \leftarrow s, V(t) \leftarrow t m p, L(t) \leftarrow L(s)$ e insira $t$ em $Q$.

O Algoritmo 1 define os rótulos como $L(t)=1$ para todo $t \in \mathcal{S}_{o}$ e $L(t)=0$ para todo $t \in \mathcal{S}_{b}($ Linhas 1 e 2). Nas Linhas 3 a 5 , para todos os caminhos triviais inicializa os mapas de predecessores $(P)$, de valores $(V)$ e o estado do vértice. Na Linha 6 , todos os candidatos a raízes (sementes) são inseridos na fila $Q$.

O laço principal (Linhas 7 a 14) calcula os caminhos ótimos, de modo que em cada iteração um caminho $\pi_{s}^{P}$ de valor mínimo é obtido em $P$ quando removemos seu último vértice $s$ de $Q$ (Linha 8). O resto das linhas (Linhas 9 a 14) calculam e propagam caminhos melhores para os vértices adjacentes, seguindo uma ordem não decrescente de valores e aplicando a seguinte regra de expansão: Se $f\left(\pi_{s}^{P} \cdot\langle s, t\rangle\right)<f\left(\pi_{t}^{P}\right)($ Linha 12), então $\pi_{t}^{P}$ é trocado por $\pi_{s}^{P} \cdot\langle s, t\rangle$ (Linha 14), ou seja, o algoritmo avalia se o caminho $\pi_{s}^{P} \cdot\langle s, t\rangle$ que alcança $t$ por meio de $s$ tem um valor menor do que o caminho atual $\pi_{t}^{P}$ em $P$ e atualiza $P(t), V(t), L(t)$ e $Q$ adequadamente. 


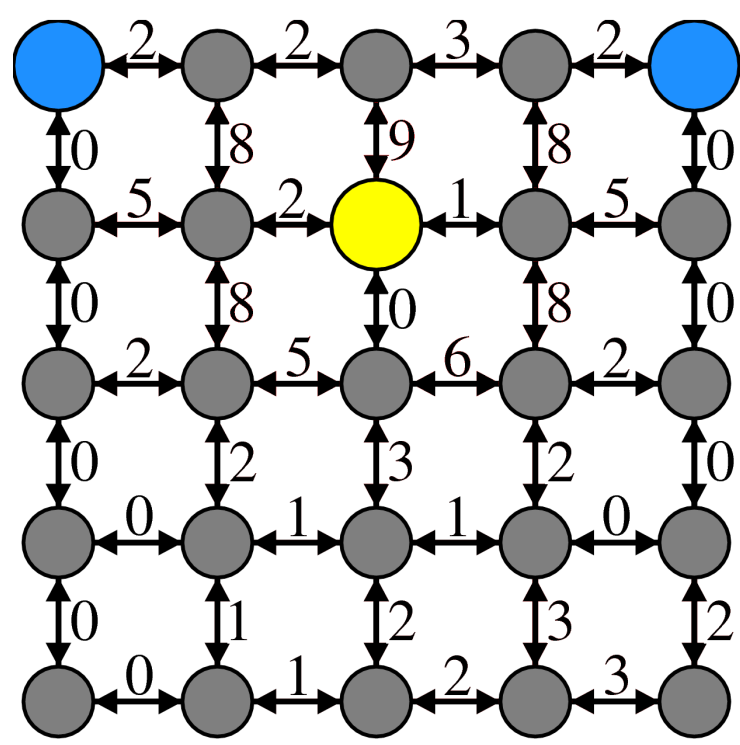

(a) Estado inicial com sementes

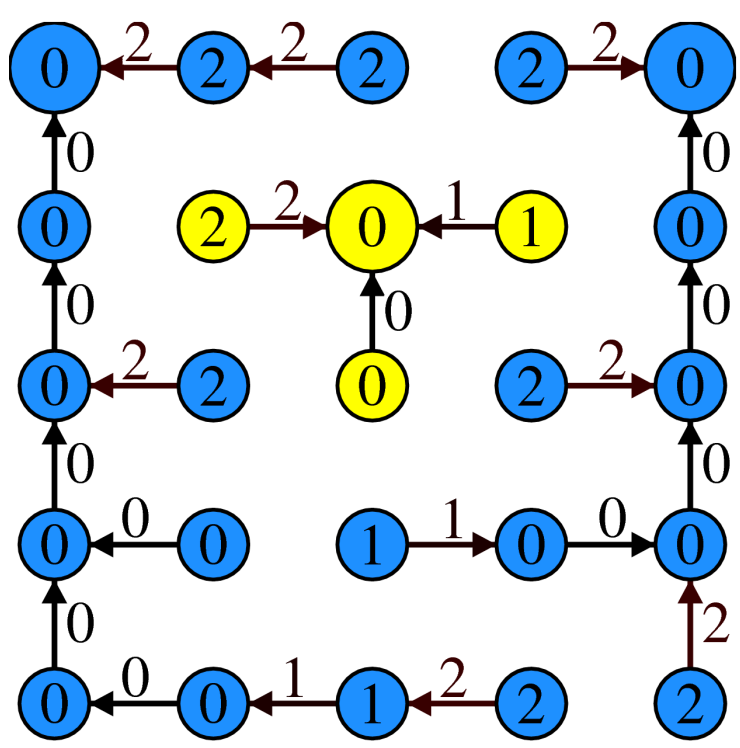

(b) Floresta de espalhamento final

Figura 2.3: Exemplo de um grafo onde foi executada a IFT com a função $f_{\max }$ Eq.(2.1.2). Em (a) temos em amarelo a semente de objeto e em azul as sementes de fundo.

\subsubsection{Zonas de empate}

Na IFT, uma floresta $P$ de caminhos ótimos pode não ser única, o que representa um problema de ambiguidade [FSL04]. Por exemplo, se duas ou mais raízes alcançam um pixel $t$ por meio de caminhos ótimos com mesmo custo, então mais de uma floresta poderia ser ótima. Empates entre caminhos $\pi_{t}$ e $\pi_{t}^{\prime}$ a partir de sementes com mesmo rótulo não representam um problema, pois eles levam ao mesmo resultado de segmentação, sendo qualquer solução satisfatória. No entanto, empates entre caminhos a partir de sementes com diferentes rótulos representam zonas de empate reais. Considerando este último caso, podemos dizer que um pixel $t$ é um pixel de empate se existem dois caminhos ótimos completos $\pi_{t}$ e $\pi_{t}^{\prime}$ tal que $L\left(R\left(\pi_{t}\right)\right) \neq$ $L\left(R\left(\pi_{t}^{\prime}\right)\right)$. Assim, uma zona de empate é um conjunto maximal $\mathcal{T}$ de pixels de empate que formam uma sub-árvore em alguma floresta de caminhos ótimos [MF09]. 


\section{Capítulo 3}

\section{Corte em Grafos Generalizado}

Agora que já conhecemos a IFT, podemos estudar melhor a variedade de problemas que ela pode resolver. Para isso, vamos introduzir o framework de Corte em Grafos Generalizado (Generalized Graph Cuts - GGC), que contém, entre outras, as formulações da $\varepsilon_{1}$ e da $\varepsilon_{\infty}$-minimização.

\subsection{Definição do framework}

Nessa seção, apresentamos apenas o framework para segmentações binárias, porém ele pode ser estendido para segmentações fuzzy, nas quais cada pixel possui diferentes graus de pertinência ao objeto. Trabalhos que tratam deste caso contínuo são discutidos em [CLLH10, CUFM12b].

Seja um grafo $G=(\mathcal{I}, \mathcal{A})$ e seja $\mathcal{X}$ o conjunto de todas as segmentações binárias possíveis. Seja $L \in \mathcal{X}$, uma segmentação possível da imagem $\mathcal{I}$. Sejam $\mathcal{S}_{o}$ e $\mathcal{S}_{b}\left(\mathcal{S}_{o} \cap \mathcal{S}_{b}=\emptyset\right)$, dois conjuntos de sementes, indiciando respectivamente, objeto e fundo. O objetivo do problema é encontrar uma segmentação que minimize uma equação de energia, sujeita às sementes dadas. No contexto deste framework, a equação de energia a ser minimizada é:

$$
\varepsilon_{q}(L)=\sqrt[q]{\sum_{(s, t) \in \mathcal{A}}(\bar{\omega}(s, t)|L(s)-L(t)|)^{q}}
$$

para algum valor $1 \leq q \leq \infty$. Nos casos particulares $q=1$ e $q=\infty$, temos respectivamente as definições das energias $\varepsilon_{1}$ e $\varepsilon_{\infty}$, que são dois casos importantes no contexto de segmentação de imagens, pois o primeiro pode ser otimizado usando o método do fluxo máximo-corte mínimo [BK04] e o segundo por meio da IFT.

Portanto, vamos focar no problema da $\varepsilon_{\infty}$-minimização.

\section{$3.2 \varepsilon_{\infty}$-minimização}

Quando estamos tratando do caso da $\varepsilon_{\infty}$-minimização, temos a seguinte equação:

$$
\varepsilon_{\infty}(L)=\max _{(s, t) \in \mathcal{A}} \bar{\omega}(s, t)|L(s)-L(t)|
$$

onde $\bar{\omega}(s, t)$ é o valor complementar do peso $\omega(s, t)$ (i.e., $\bar{\omega}(s, t)=\max _{(x, y) \in \mathcal{A}} \omega(x, y)-\omega(s, t)$ ).

$\mathrm{Na}$ IFT, quando consideramos a segmentação baseada nos dois conjuntos de semente, definidos anteriormente, as rotulações obtidas pelas funções de conexidade $f_{\max }$ (Eq. 2.1.2) (com a política LIFO de desempate), $f_{w}$ (Eq. 3.2.2) e $f_{\max }^{b k g}$ (Eq. 3.2.3), entre outras, são soluções ótimas para o problema da $\varepsilon_{\infty^{-}}$ minimização, no caso de grafos não dirigidos [CUFM12a].

$$
\begin{aligned}
f_{\omega}(\langle t\rangle) & = \begin{cases}0 & \text { se } t \in \mathcal{S}_{o} \cup \mathcal{S}_{b} \\
+\infty & \text { caso contrário }\end{cases} \\
f_{\omega}\left(\pi_{s} \cdot\langle s, t\rangle\right) & =\omega(s, t)
\end{aligned}
$$




$$
\begin{aligned}
f_{\max }^{b k g}(\langle t\rangle) & = \begin{cases}0 & \text { se } t \in \mathcal{S}_{o} \cup \mathcal{S}_{b} \\
+\infty & \text { caso contrário }\end{cases} \\
f_{\max }^{b k g}\left(\pi_{r \rightsquigarrow t}=\pi_{r \rightsquigarrow s} \cdot\langle s, t\rangle\right) & = \begin{cases}\max \left\{f_{\max }^{b k g}\left(\pi_{r \rightsquigarrow s}\right), 2 \cdot \omega(s, t)+1\right\} & \text { se } r \in \mathcal{S}_{o} \\
\max \left\{f_{\max }^{b k g}\left(\pi_{r \rightsquigarrow s}\right), 2 \cdot \omega(s, t)\right\} & \text { se } r \in \mathcal{S}_{b}\end{cases}
\end{aligned}
$$

Portanto, as segmentações resultantes nos dão uma solução ótima global com respeito a função de energia da borda de corte [CUFM12a, CLLH10]. Eles minimizam a medida do corte em grafos $\varepsilon_{\infty}$ (Eq. 3.1.1) sujeita às restrições de rotulação das sementes.

Note que as funções $f_{m a x}^{b k g}$ e $f_{\omega}$ não pertencem à classe das funções suaves, portanto nesse caso as segmentações resultantes são ótimas exclusivamente do ponto de vista da energia $\varepsilon_{\infty}$, podendo a floresta de espalhamento $P$ não ser ótima no que se refere aos caminhos calculados. Um exemplo disso é ilustrado na figura a seguir.

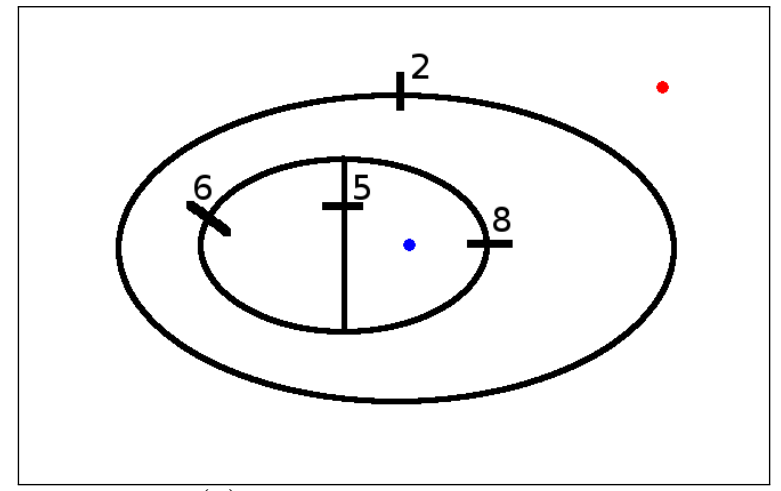

(a) Imagem com sementes

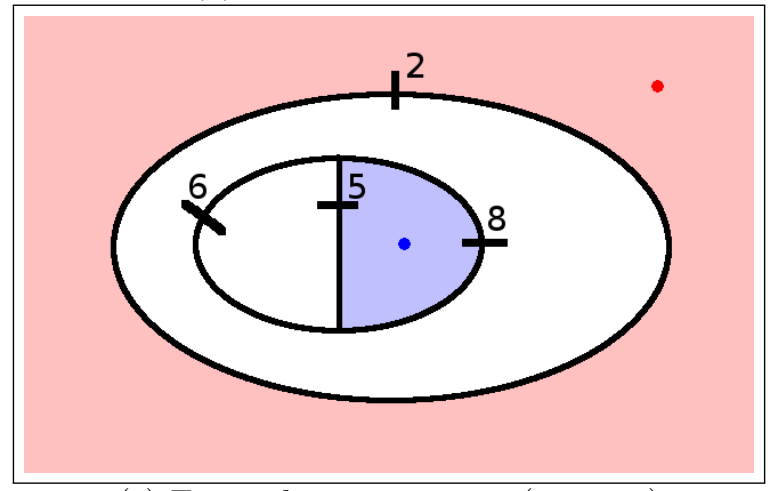

(c) Etapa de segmentação (pesos $\omega$ )

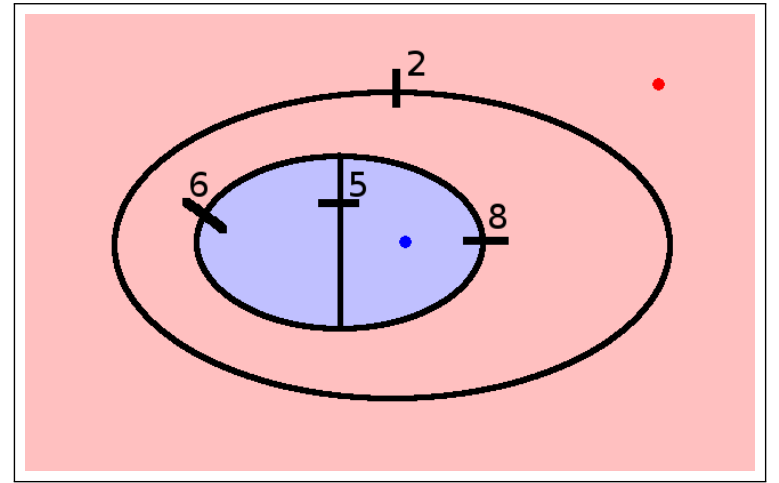

(e) Segmentação final (pesos $\omega)$

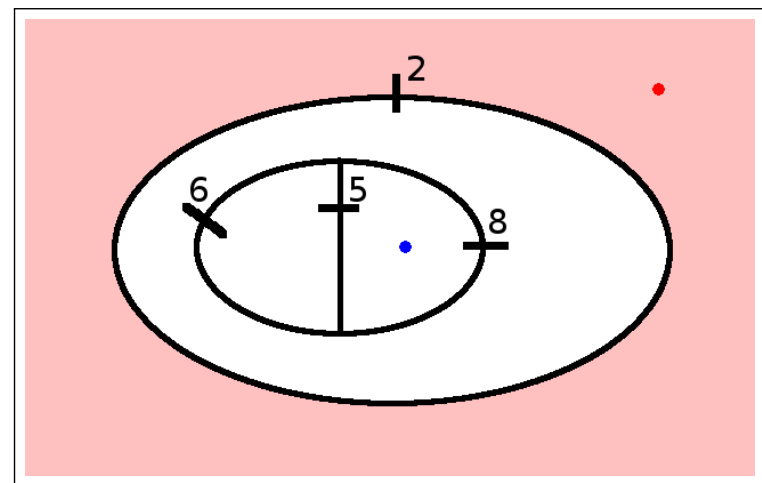

(b) Etapa de segmentação (pesos $\omega)$

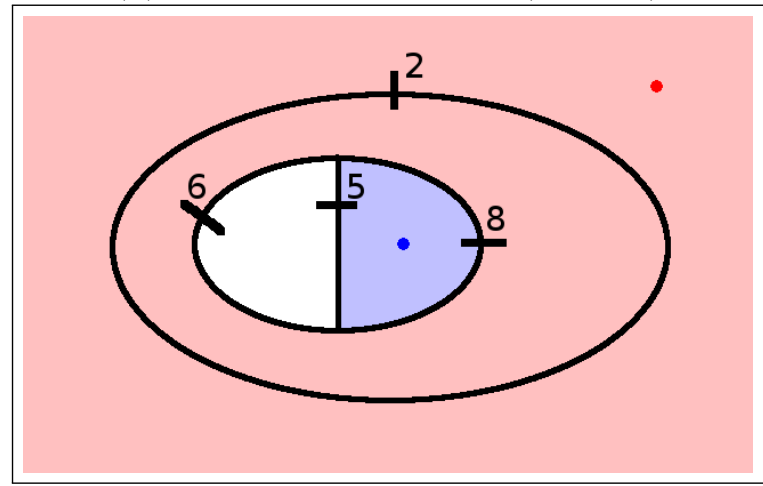

(d) Etapa de segmentação (pesos $\omega$ )

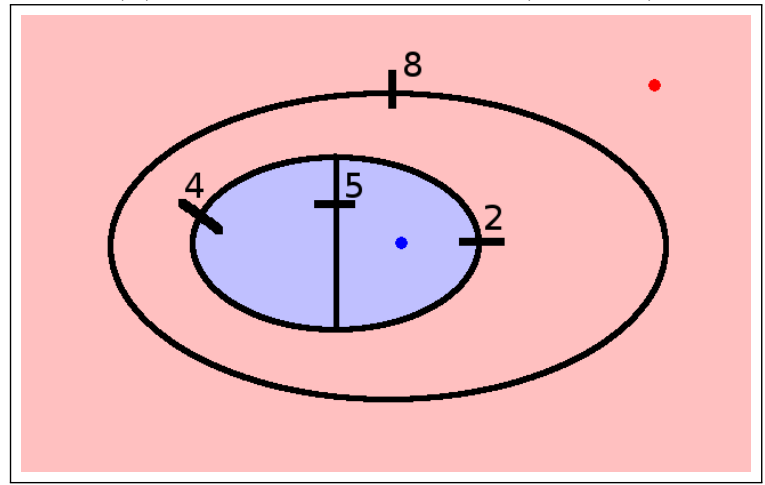

(f) Segmentação final (valores $\bar{\omega}$ )

Figura 3.1: Exemplo de segmentação no qual é possível ver a otimalidade em relação à energia $\varepsilon_{\infty}$ de uma segmentação. Note que em $(f)$, os valores $\bar{\omega}$ nos mostram que a segmentação resultante é ótima em respeito à energia $\varepsilon_{\infty}$. 


\section{Capítulo 4}

\section{Convexidade Geodésica em Estrela}

A importância das restrições de forma na IFT se deve ao fato das segmentações geradas serem irregulares, isso faz com que medidas de regularização sejam interessantes. Uma dessas medidas é a imposição de restrições de forma ao objeto, de modo a filtrar formas indesejadas.

Antes de apresentar a nossa restrição da faixa de restrição geodésica, primeiramente é importante apresentar a restrição de convexidade geodésica em estrela. Entre os motivos pelos quais ela é interessante, destacamos o fato de ser uma restrição recente $\left[\mathrm{GRC}^{+} 10\right]$, ter algumas características similares à faixa de restrição geodésica, que será apresentada no próximo capítulo, e pelo fato de ser possível combina-la com a faixa de restrição geodésica, de modo a obter maior flexibilidade na escolha de restrições.

Este capítulo é baseado no capítulo homônimo de [Man14].

\subsection{Convexidade em estrela}

Um objeto atende a convexidade em estrela em relação a um ponto central $c$ se para todo ponto $p$ do objeto todos os pontos do segmento de reta ligando $c$ a $p$ também fazem parte do objeto. Note que qualquer forma convexa também é convexa em forma de estrela, porém nem toda forma convexa em forma de estrela é convexa, fazendo com que a restrição de convexidade em estrela seja mais flexível, como ilustramos na Figura 4.1.

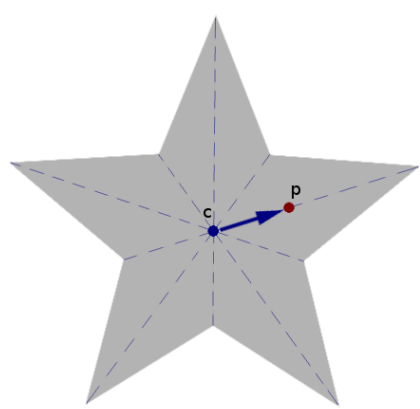

(a) Forma convexa em estrela.

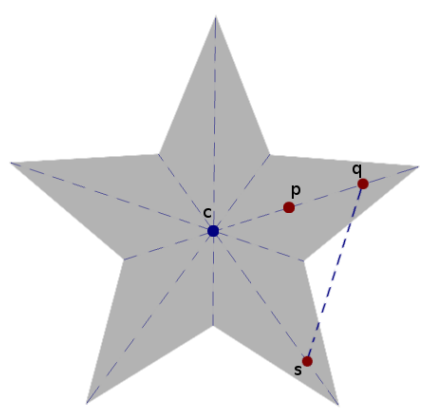

(b) Forma não convexa.

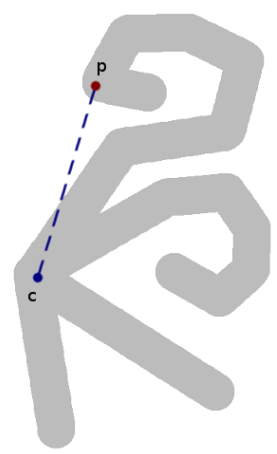

(c) Forma não convexa em estrela.

Figura 4.1: Exemplos de formas onde vemos as restrições de convexidade. Note que em (a) e (b), ilustramos como uma mesma forma pode ser convexa em estrela e não convexa na definição tradicional.

A convexidade em estrela pode ser interpretada como uma restrição de visibilidade, isto é, um objeto é convexo em estrela em relação ao ponto central $c$, se para todo ponto $p$ no objeto, $p$ é visível a $c$ via o segmento de reta interligando eles, o qual também deve fazer parte do objeto (o fundo é considerado como um obstáculo que obstrui a "luz" emitida por $p$ até o observador $c$ ). Uma possível extensão para vários centros de estrela (Figura 4.2(a)), considera que os pontos do objeto devem ser visíveis em relação a pelo menos um dos centros. Porém esta definição é computacionalmente intratável, estando relacionada a um problema NP-completo [[GRC $\left.\left.{ }^{+} 10\right]\right]$. Assim, uma alternativa de solução computacionalmente viável é considerar o argumento da distância mais curta (Figura 4.2(b)). Essa alternativa pode ser definida em 
uma versão discreta no domínio da imagem $\hat{I}$ (Figura 4.2(c)), onde os segmentos de reta correspondem a caminhos no grafo derivado da imagem.

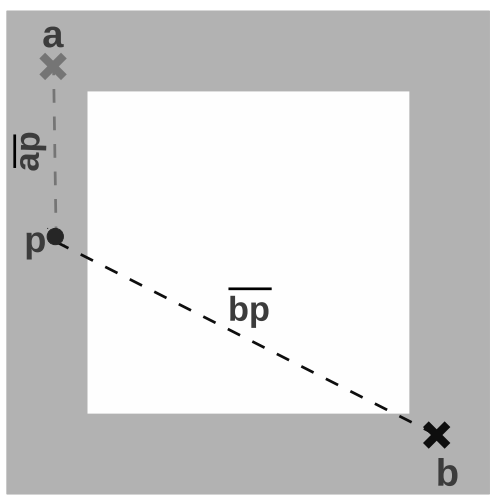

(a)

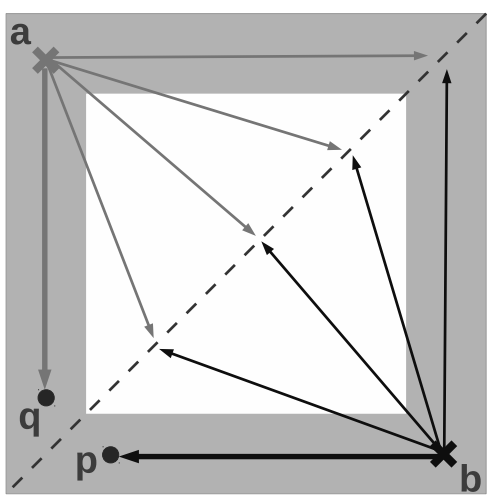

(b)

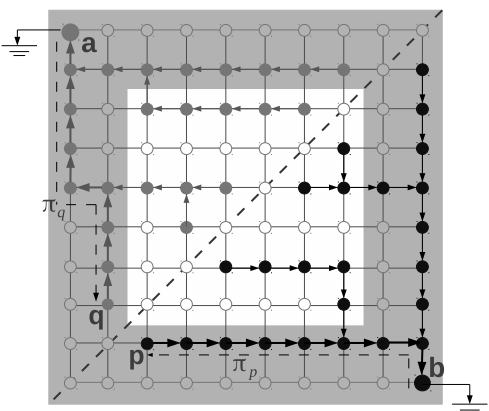

(c)

Figura 4.2: Considere um objeto $\mathcal{O}$ (área sombreada), com o conjunto de centros de estrela $C=\{a, b\}$. Em (a) temos o argumento de visibilidade: Qualquer ponto $p \in \mathcal{O}$ deve ser visível ao menos por um dos centros de estrela (por exemplo, $p$ é visivel através de $\overline{a p}$, embora este não seja visivel através de $\overline{b p}$ ). Em (b) temos o argumento da distancia mais curta: Cada ponto é analisado exclusivamente em relação a seu centro mais próximo (por exemplo, $q$ está mais próximo de a, e p mais próximo de b). Em (c) temos uma versão discreta pelo caminho mais curto no grafo de imagem.

\subsection{Segmentação com restrições de convexidade geodésica em es- trela}

Nós consideramos os centros de estrela como as sementes internas selecionadas pelo usuário e os segmentos de reta como os caminhos ligando cada ponto do objeto ao centro de estrela mais próximo formando uma floresta de caminhos de custo geodésico $\left(f_{\text {sum }}\right)$ mínimo da IFT.

Assim, nós definimos os pesos das arestas $\delta(s, t)$ na regra de extensão de caminho para $f_{\text {sum }}$ (Equação 2.1.1) como:

$$
\delta(s, t)=[\omega(s, t)+1]^{\beta}-1+\|t-s\|
$$

onde $\|t-s\|$ é a distância Euclideana entre os pixels $s$ e $t$, e $\beta$ controla a topologia da floresta. Para valores muito pequenos de $\beta(\beta \approx 0.0), \delta(s, t)$ aproxima-se de $\|t-s\|$ e a topologia da floresta torna-se similar à floresta de caminhos ótimos Euclideanos (Figura 4.3(a)). Para valores maiores, $[\omega(s, t)+1]^{\beta}$ domina a expressão, e quanto maior for a dissimilaridade baseada em intensidade, maior será a sua influência sobre os resultados (Figuras 4.3(b), 4.3(c) e 4.3(d)). Logo, dependendo de como são configurados os pesos das arestas tem-se caminhos que seguem raios euclidianos ou que se adaptam as formas presentes na imagem fazendo curvas, de acordo com o parâmetro de controle $\beta$.

A Figura 4.4 mostra como o parâmetro $\beta$ afeta a floresta para $f_{\text {sum }}$, e sua correspondente segmentação com restrições de forma. Claramente, valores mais baixos de $\beta$ impõem mais regularização para a borda do objeto, enquanto valores mais elevados permitem um melhor ajuste para protuberâncias e saliências na borda. 


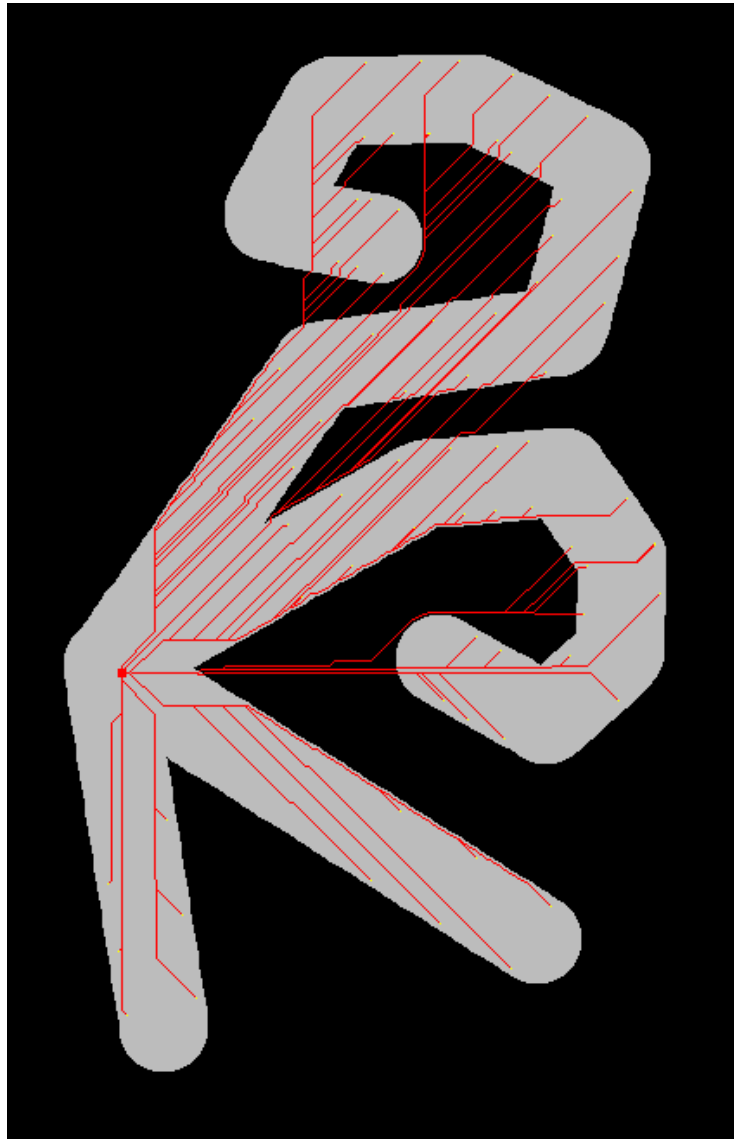

(a) $\beta=0.1$

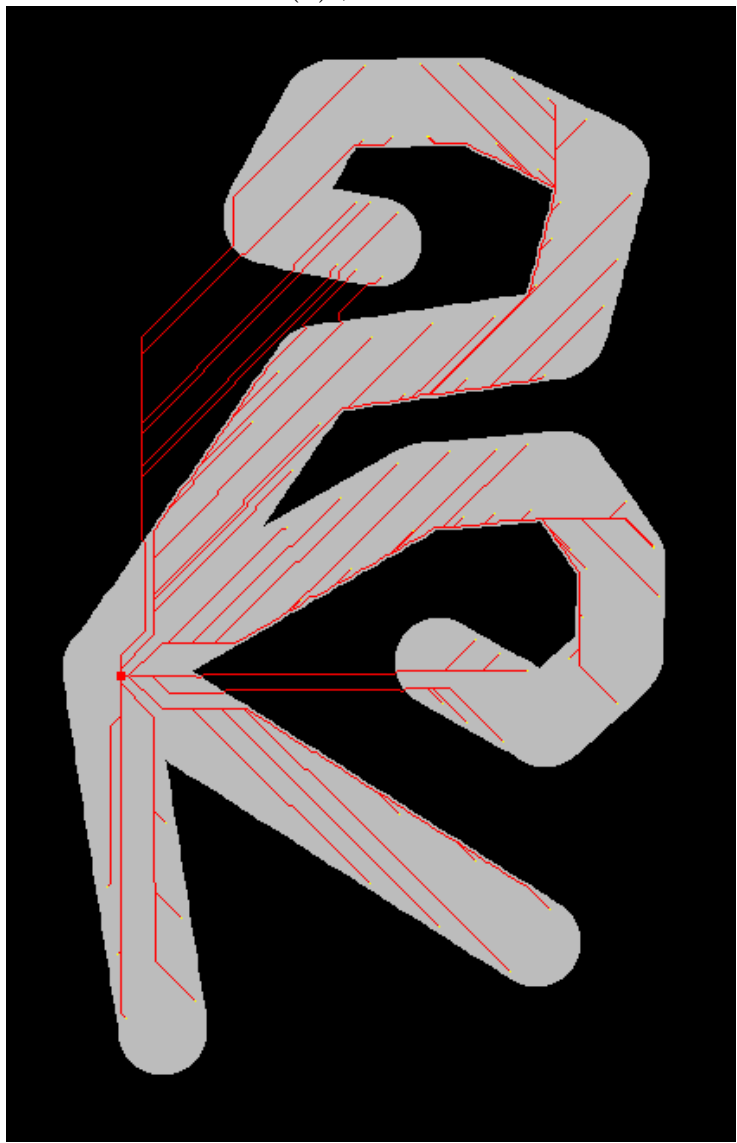

(c) $\beta=0.5$

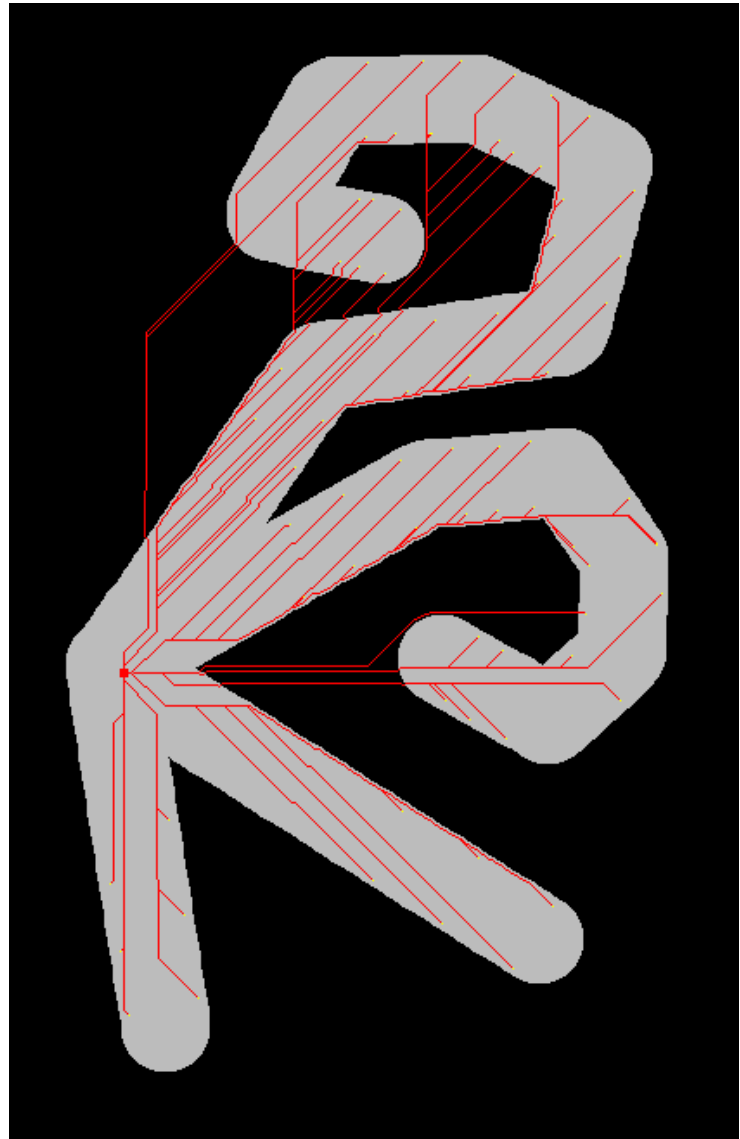

(b) $\beta=0.3$

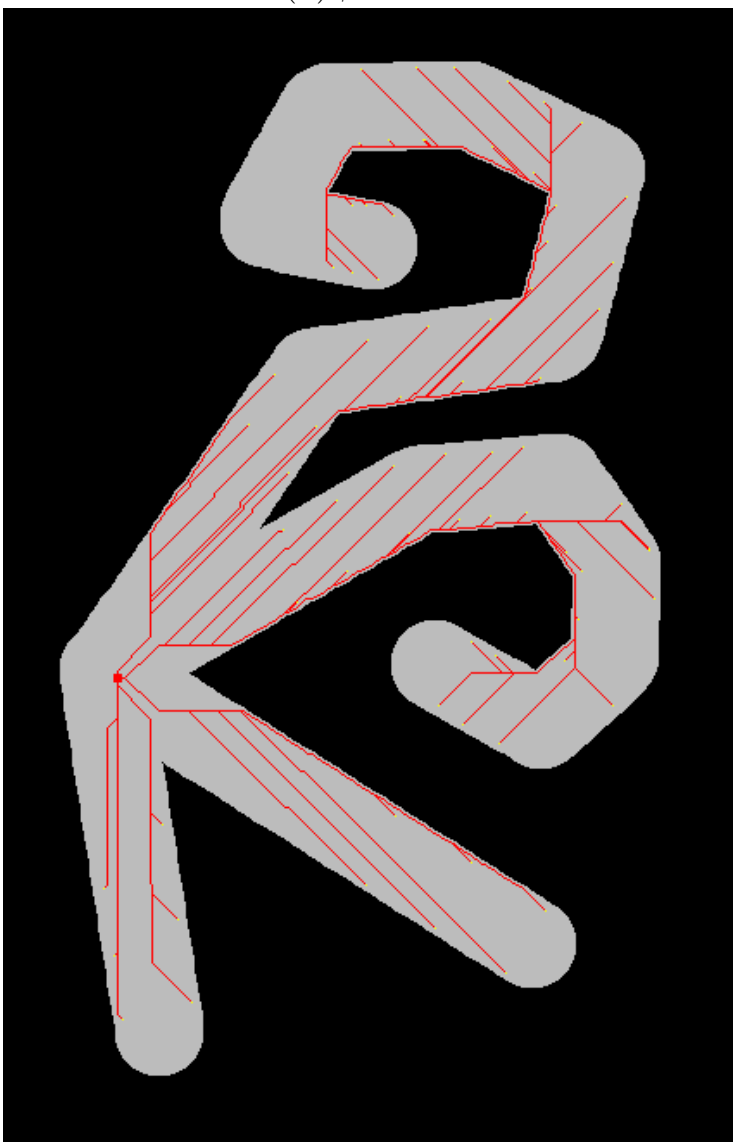

(d) $\beta=0.7$

Figura 4.3: Efeito do parâmetro de potência $\beta$ sobre a topologia da floresta: A floresta de caminhos ótimos de $f_{\text {sum }}$ para diferentes valores de $\beta$. Podemos visualizar que quanto maior o valor de $\beta$, mais os caminhos se adaptam às características da imagem. 


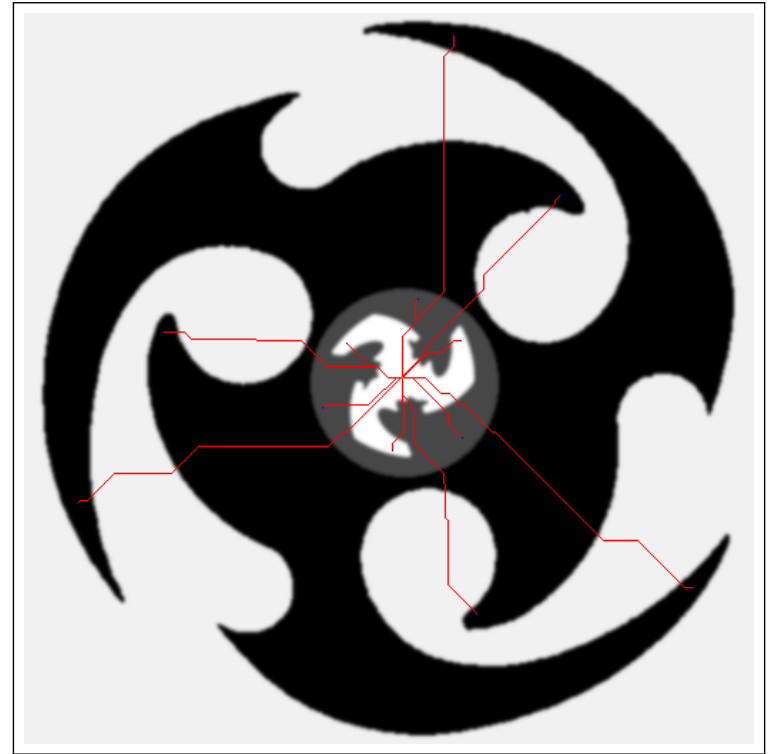

(a) Floresta de caminhos ótimos $\operatorname{com} \beta=0.1$

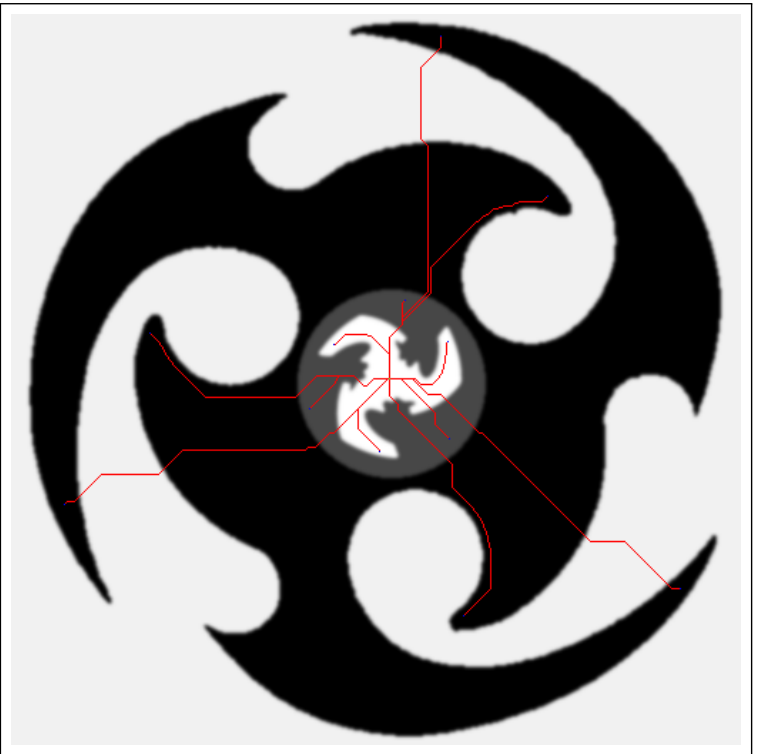

(c) Floresta de caminhos ótimos $\operatorname{com} \beta=0.3$

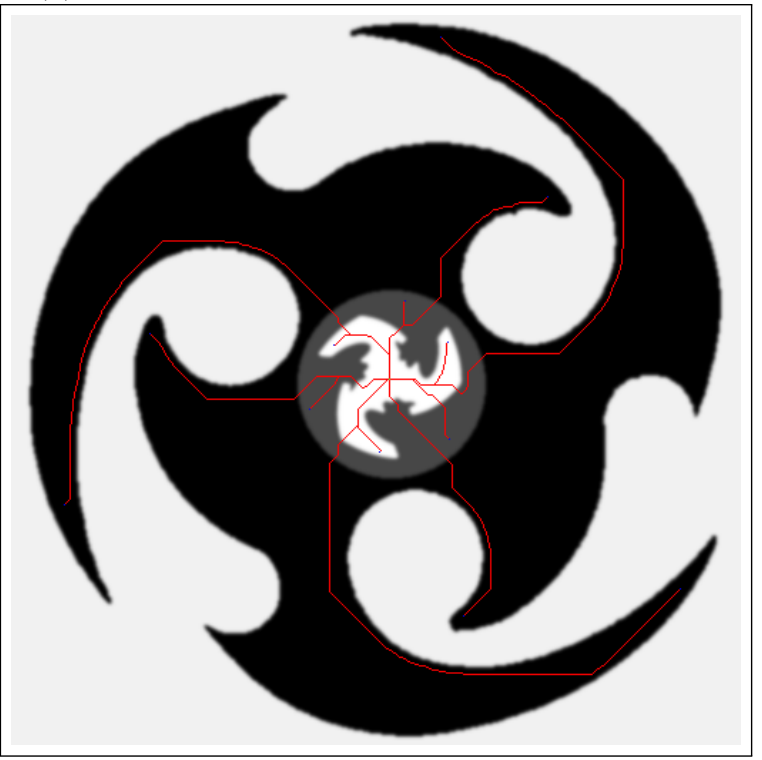

(e) Floresta de caminhos ótimos $\operatorname{com} \beta=0.7$

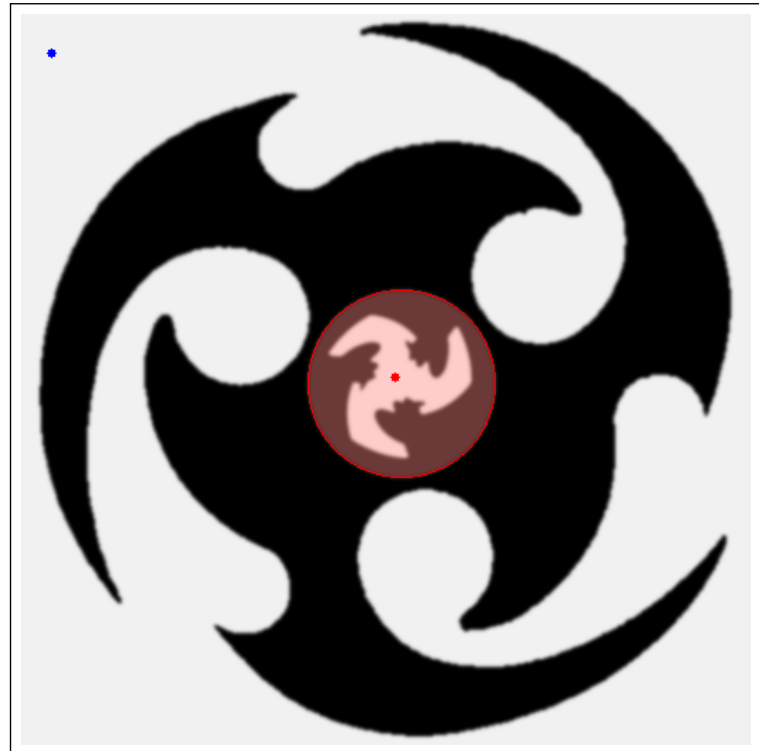

(b) Segmentação com $\beta=0.1$

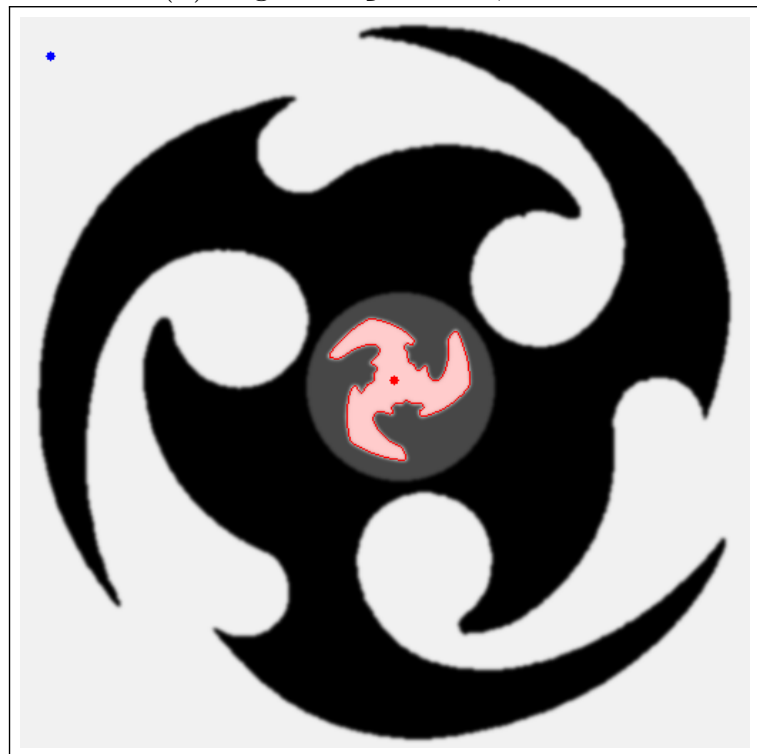

(d) Segmentação $\operatorname{com} \beta=0.3$

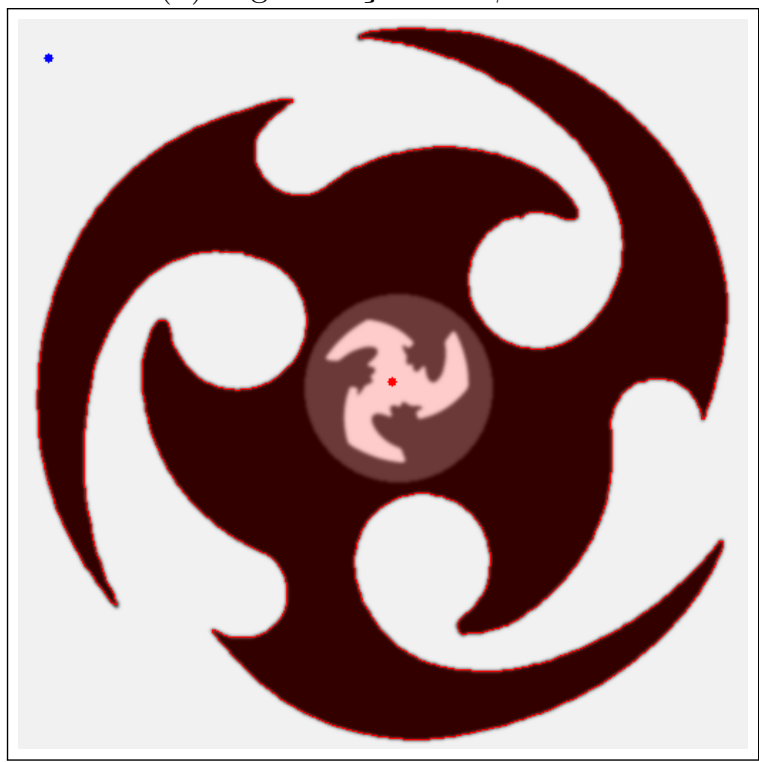

(f) Segmentação $\operatorname{com} \beta=0.7$

Figura 4.4: Efeitos do parâmetro de potência $\beta$ sobre a topologia da floresta de caminhos ótimos e na segmentação resultante. Novamente podemos notar como a adaptabilidade controlada pelo parâmetro $\beta$ influencia o resultado final da segmentação. 


\section{Capítulo 5}

\section{Transformada Imagem-Floresta com Orientação de Bordas - OIFT}

Neste capítulo vamos mostrar como a IFT pode ser usada quando modelamos a imagem por meio de grafos dirigidos, resultando em um método no qual é possível segmentar imagens favorecendo bordas com transições que variam do escuro para o claro ou vice-versa.

Quando trabalhamos com imagens que estão modeladas como grafos dirigidos, é importante notar que cada segmentação define dois tipos distintos de corte, um deles definido pelos arcos que estão atravessando o corte no sentido fundo-objeto e outro que é definido pelos arcos que estão atravessando o corte no sentido objeto-fundo. Sendo assim, temos duas energias distintas, que geram resultados diferentes quando são otimizadas.

\subsection{Definição}

Como visto em [MM13a, MM14], quando tratamos nossa imagem como um grafo dirigido (digrafo) $G=(V, A)$, precisamos levar em conta dois tipos de corte na borda dos objetos, um deles formado pelos arcos que apontam para dentro do objeto (inner cut $-C_{i}$ ) e outro formado pelos arcos que apontam para fora dele (outer cut - $C_{o}$ ), definidos a seguir:

$$
\begin{aligned}
& C_{i}(L)=\{(s, t) \in \mathcal{A} \mid L(s)=0, L(t)=1\} \\
& C_{o}(L)=\{(s, t) \in \mathcal{A} \mid L(s)=1, L(t)=0\}
\end{aligned}
$$

Estes cortes têm uma energia associada, que os métodos de segmentação por meio de otimização de energia devem levar em conta, sendo uma para $C_{i}$ e outra para $C_{o}$ :

$$
\begin{aligned}
& E_{i}(L)=\max _{(s, t) \in C_{i}(L)} \bar{\omega}(s, t) \\
& E_{o}(L)=\max _{(s, t) \in C_{o}(L)} \bar{\omega}(s, t)
\end{aligned}
$$

Neste caso, usamos uma digrafo onde o peso $\omega(s, t)$ dos arcos é uma combinação entre a medida de dissimilaridade $\psi(s, t)$, que é usada no caso não dirigido, multiplicada por uma fator de orientação, como vemos na definição a seguir e ilustrado na Figura 5.1:

$$
\omega(s, t)=\left\{\begin{array}{l}
\psi(s, t) \cdot(1+\alpha), \text { se } I(s)>I(t), \\
\psi(s, t) \cdot(1-\alpha), \text { caso contrário. }
\end{array}\right.
$$




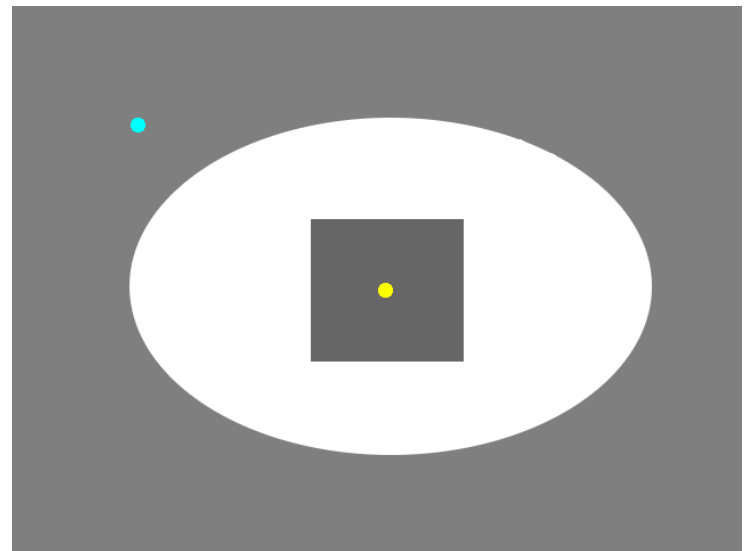

Imagem com sementes

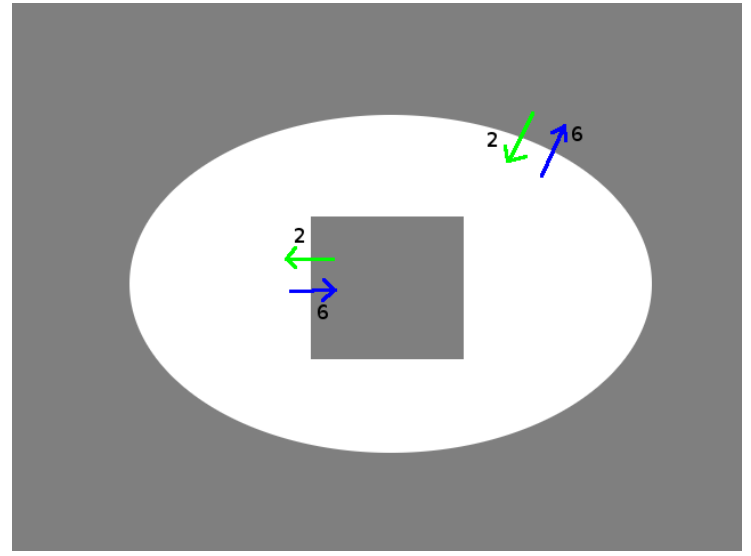

Custos $\omega$

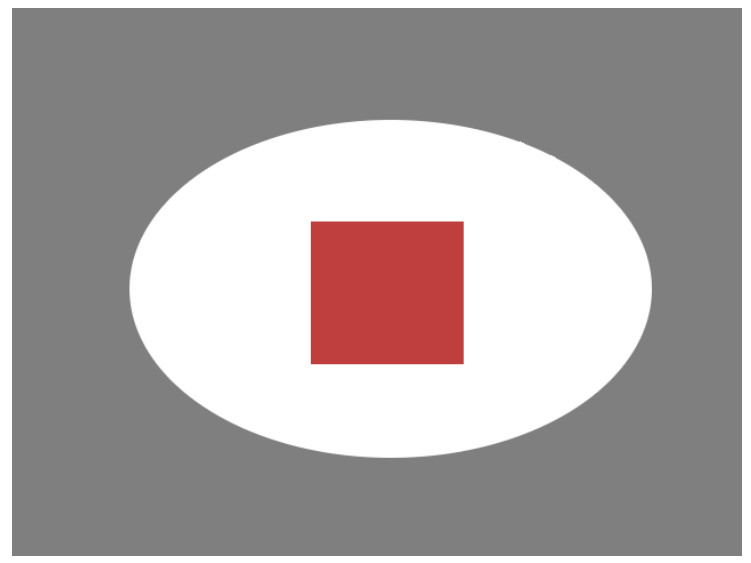

Segmentação otimizando $C_{i}$ Note que $E_{i}=4$ e $E_{o}=8$

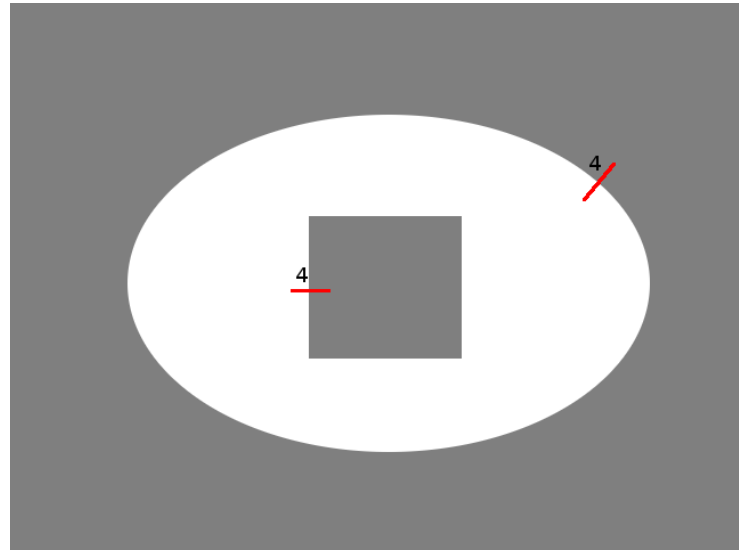

Custo $\Psi$

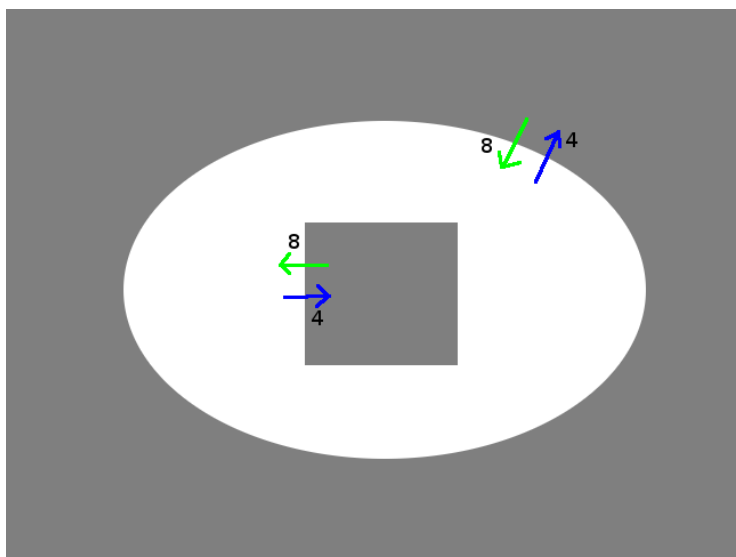

Custos $\bar{\omega}$

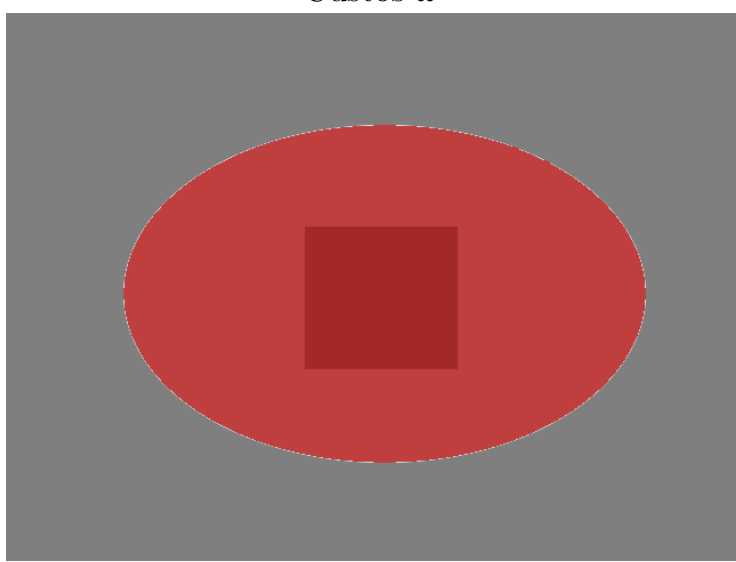

Segmentação otimizando $C_{o}$ Note que $E_{i}=8$ e $E_{o}=4$

Figura 5.1: Exemplo ilustrativo da OIFT segmentando um $C_{i}$ (escuro para claro) e $C_{o}$ (claro para escuro).

\subsection{Orientação de bordas na IFT}

Utilizando a IFT podemos usar as seguintes funções de custo de modo a obter segmentações que otimizem as energias definidas acima e consequentemente favorecer transições de áreas claras para escuras ou escuras para claras, como vemos na Figura 5.2.

$$
f_{i, \max }^{b k g}(\langle t\rangle)=\left\{\begin{array}{l}
-1, \text { se } t \in \mathcal{S}_{o} \cup \mathcal{S}_{b} \\
+\infty, \text { caso contrário }
\end{array} \quad f_{i, \max }^{b k g}\left(\pi_{r \rightsquigarrow s}\langle s, t\rangle\right)=\left\{\begin{array}{l}
\max \left\{f_{i, \max }^{b k g}\left(\pi_{r \rightsquigarrow s}\right), 2 \cdot \omega(t, s)+1\right\}, \text { se } r \in \mathcal{S}_{o} \\
\max \left\{f_{i, \max }^{b k g}\left(\pi_{r \rightsquigarrow s}\right), 2 \cdot \omega(s, t)\right\}, \text { se } r \in \mathcal{S}_{b}
\end{array}\right.\right.
$$




$$
\begin{aligned}
& f_{o, \max }^{b k g}(\langle t\rangle)=\left\{\begin{array}{l}
-1, \text { se } t \in \mathcal{S}_{o} \cup \mathcal{S}_{b} \\
+\infty, \text { caso contrário }
\end{array} \quad f_{o, \text { max }}^{b k g}\left(\pi_{r \rightsquigarrow s}\langle s, t\rangle\right)=\left\{\begin{array}{l}
\max \left\{f_{o, \max }^{b k g}\left(\pi_{r \rightsquigarrow s}\right), 2 \cdot \omega(s, t)+1\right\}, \text { se } r \in \mathcal{S}_{o} \\
\max \left\{f_{o, \max }^{b k g}\left(\pi_{r \rightsquigarrow s}\right), 2 \cdot \omega(t, s)\right\}, \text { se } r \in \mathcal{S}_{b}
\end{array}\right.\right. \\
& f_{i, \omega}(\langle t\rangle)=\left\{\begin{array}{l}
-1, \text { se } t \in \mathcal{S}_{o} \cup \mathcal{S}_{b} \\
+\infty, \text { caso contrário }
\end{array} \quad f_{i, \omega}\left(\pi_{r \rightsquigarrow s}\langle s, t\rangle\right)=\left\{\begin{array}{l}
\omega(t, s), \text { se } r \in \mathcal{S}_{o} \cup \mathcal{S}_{b} \\
\omega(s, t), \text { se } r \in \mathcal{S}_{b} \cup \mathcal{S}_{b}
\end{array}\right.\right. \\
& f_{o, \omega}(\langle t\rangle)=\left\{\begin{array}{l}
-1, \text { se } t \in \mathcal{S}_{o} \cup \mathcal{S}_{b} \\
+\infty, \text { caso contrário }
\end{array} \quad f_{o, \omega}\left(\pi_{r \rightsquigarrow s}\langle s, t\rangle\right)=\left\{\begin{array}{l}
\omega(s, t), \text { se } r \in \mathcal{S}_{o} \cup \mathcal{S}_{b} \\
\omega(t, s), \text { se } r \in \mathcal{S}_{b} \cup \mathcal{S}_{b}
\end{array}\right.\right.
\end{aligned}
$$

Teorema 1. Para dois conjuntos de sementes $\mathcal{S}_{o}$ e $\mathcal{S}_{b}$, qualquer floresta de espalhamento computada pelo algoritmo da IFT usando a função $f_{o, \max }(5.2 .2)$ ou $f_{o, \omega}$ (5.2.4) define um corte ótimo que minimiza a energia $E_{o}(L)$ assim como as funções $f_{i, \max }(5.2 .1)$ e $f_{i, \omega}(5.2 .3)$ geram cortes que minimizam $E_{i}(L)$, dentre todas as possiveis segmentações que satisfazem as restriçôes dadas.

A prova deste teorema é apresentada nos artigos [MM13a, MM14].

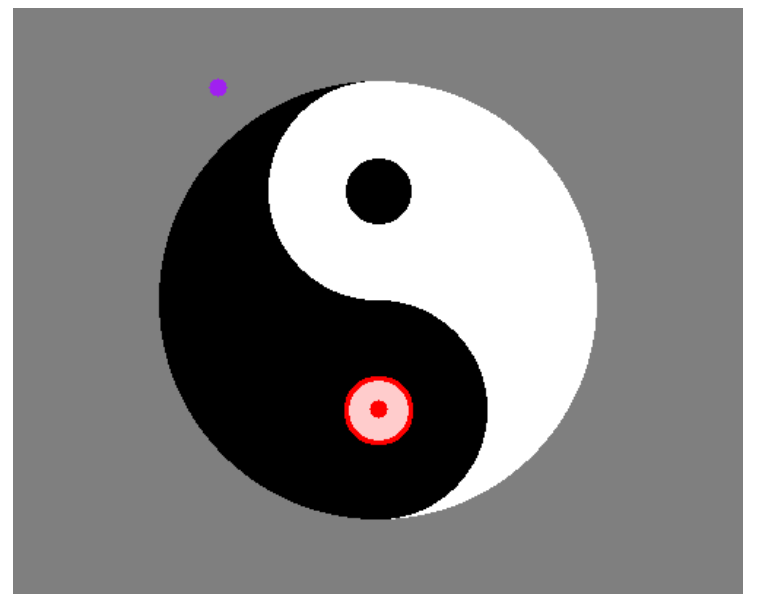

(a) OIFT com $f_{o, \max }^{b k g}$

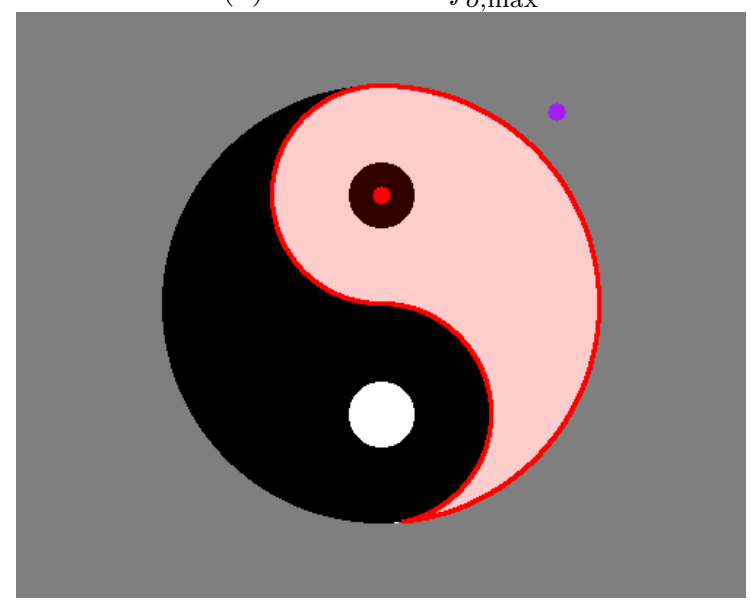

(c) OIFT com $f_{o, \max }^{b k g}$

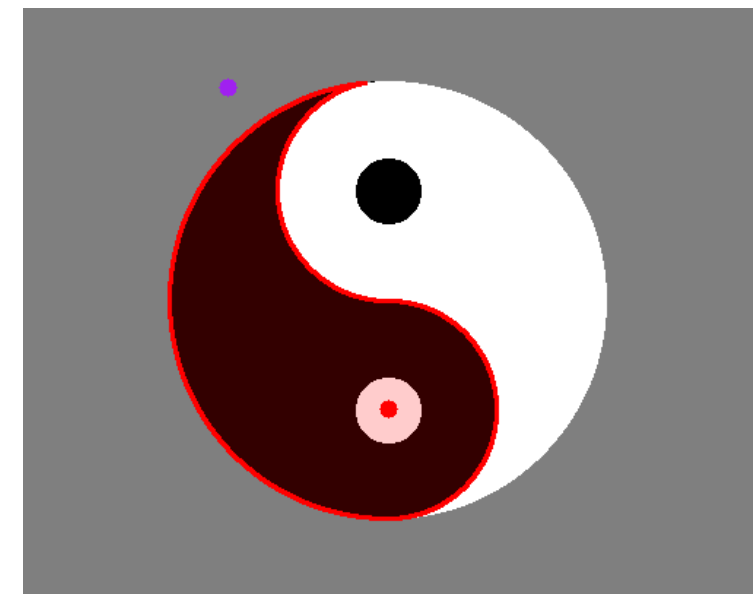

(b) OIFT com $f_{i, \text { max }}^{b k g}$

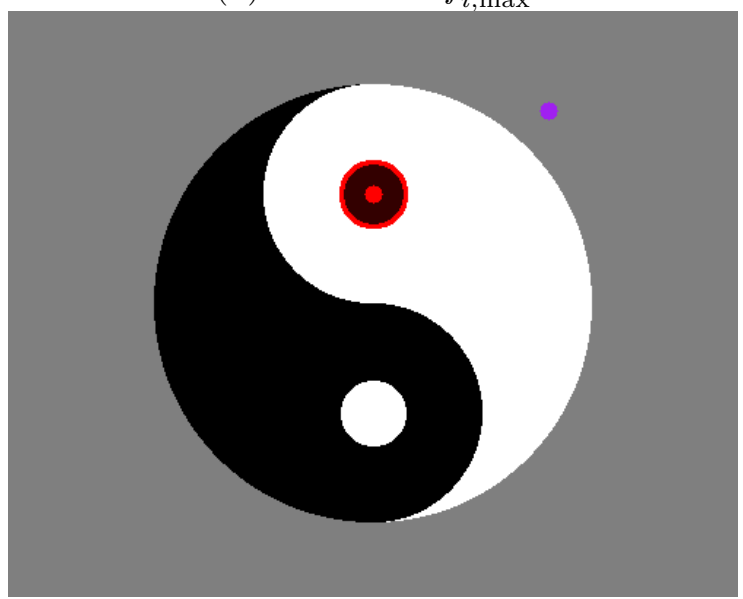

(d) OIFT com $f_{i, \text { max }}^{b k g}$

Figura 5.2: Exemplo de uso da OIFT, onde podemos determinar qual área da imagem deve ser segmentada de acordo com a transição desejada. Nota-se que podemos segmentar elementos distintos da imagem com o mesmo conjunto de sementes, alterando apenas a energia a ser otimizada. 


\section{Capítulo 6}

\section{Faixa de Restrição Geodésica}

Conhecendo a IFT, OIFT e a GSC, podemos apresentar a nova restrição de forma, que batizamos de Faixa de Restrição Geodésica, que consiste em limitar as disposições espaciais entre pontos da borda do objeto dentro de uma faixa de custo geodésico, de modo a impedir que a segmentação encontrada seja muito irregular. Controlamos a largura dessa faixa por meio de um parâmetro fixo, sendo assim possível ajustar a restrição.

\subsection{Faixa de Restrição}

A ideia principal da faixa de restrição consiste em fixar uma variação máxima de custo geodésico entre os pontos da borda do objeto a ser segmentado, de modo a impedir que o contorno gerado pela segmentação seja irregular. Obtemos este efeito por meio de um processo que podemos dividir em duas etapas:

1. Geração de um mapa de custos geodésico $C$.

Assim como feito na restrição GSC [GRC ${ }^{+} 10$, MJM13], esta primeira etapa consiste em calcular uma floresta de caminhos ótimos cujas raízes são as sementes de objeto definidas inicialmente. Isto é feito por meio de uma IFT com uma função de custo aditiva (geodésica) Eq. (2.1.1). Esta floresta é armazenada em um mapa de predecessores $P$ e os valores dos caminhos são armazenados em um mapa de custos $C$.

Neste processo utilizamos a função geodésica da distância espacial dos pixels vizinhos $(\beta=0$ na Eq. 4.2.1), de modo a obter uma interpretação física mais natural da faixa. Outras funções de custo podem ser utilizadas no lugar da geodésica, levando consequentemente a interpretações distintas sobre a faixa. O único requisito teórico é que o valor $C(t)$ dos pixels ao longo do caminho deve ser uma função não decrescente.

2. Segmentação com a faixa de restrição.

Nesta etapa é realizada a segmentação de fato. Considere o conjunto de pixels do objeto $\mathcal{O}=$ $\{t \in \mathcal{I} \mid L(t)=1\}$ e de pixels em sua borda $\mathcal{B}(\mathcal{O})=\{t \in \mathcal{O} \mid \exists s \in \mathcal{A}(t)$ tal que $s \notin \mathcal{O}\}$. A definição da nova restrição de borda é dada a seguir:

Definição 1 (Faixa de Restrição Geodésica - Geodesic Band Constraint - GBC). Um objeto $\mathcal{O}$ satisfaz a faixa de restrição geodésica com tamanho $\Delta$ se $\forall t \in \mathcal{O}$ vale que $C(t) \leq \min _{s \in \mathcal{B}(\mathcal{O})} C(s)+\Delta$.

A GBC restringe os pixels da borda em $\mathcal{B}(\mathcal{O})$ de modo que eles estejam dentro de uma faixa de largura fixa $\Delta$, impedindo que a borda encontrada tenha variações maiores que este valor, com isso regularizando sua forma. A seguir, mostraremos um algoritmo que encontra uma segmentação que minimiza a energia $\varepsilon_{\infty}$ (Eq. 3.2.1) entre todos os resultados possíveis que satisfazem a GBC, que chamaremos de IFT com Faixa de Restrição Geodésica (Geodesic Band Constraint IFT - GBC-IFT). Durante o processo, a faixa muda de posição, permitindo sua adaptação ao conteúdo da imagem (Figura 6.1). Note que apenas a sua posição é alterada enquanto a largura se mantém fixa.

\subsection{Algoritmos}

Nesta seção, mostraremos o algoritmo que realiza a segunda parte do processo, isto é, a parte que efetivamente aplica a GBC durante a segmentação da imagem que será chamado de GBC-IFT, assim como alguns algoritmos auxiliares que são utilizados durante o processo. 


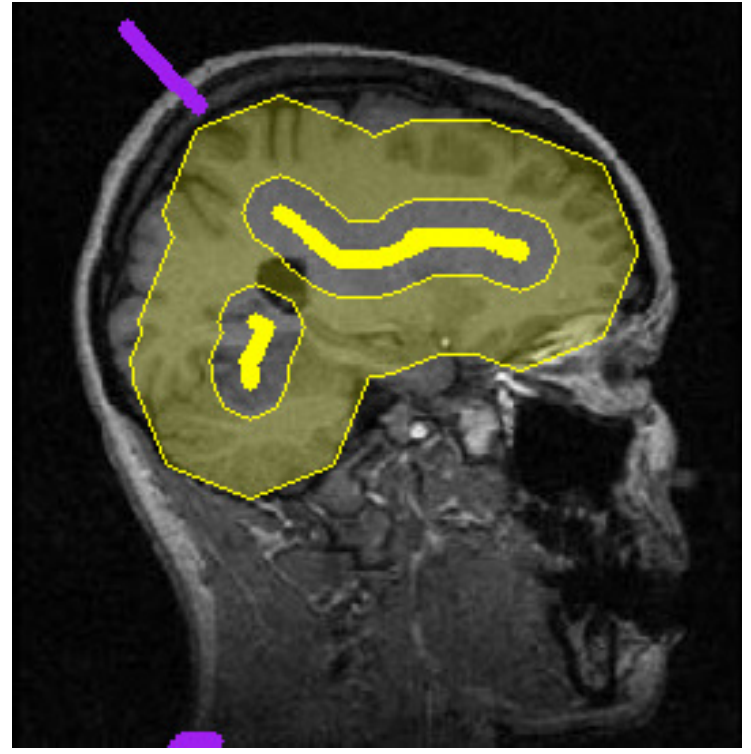

(a)

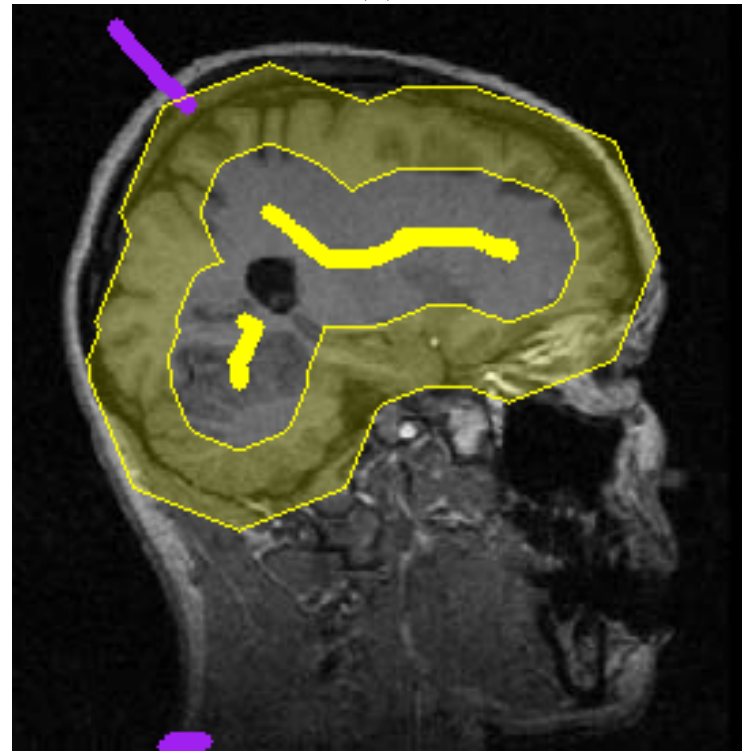

(c)

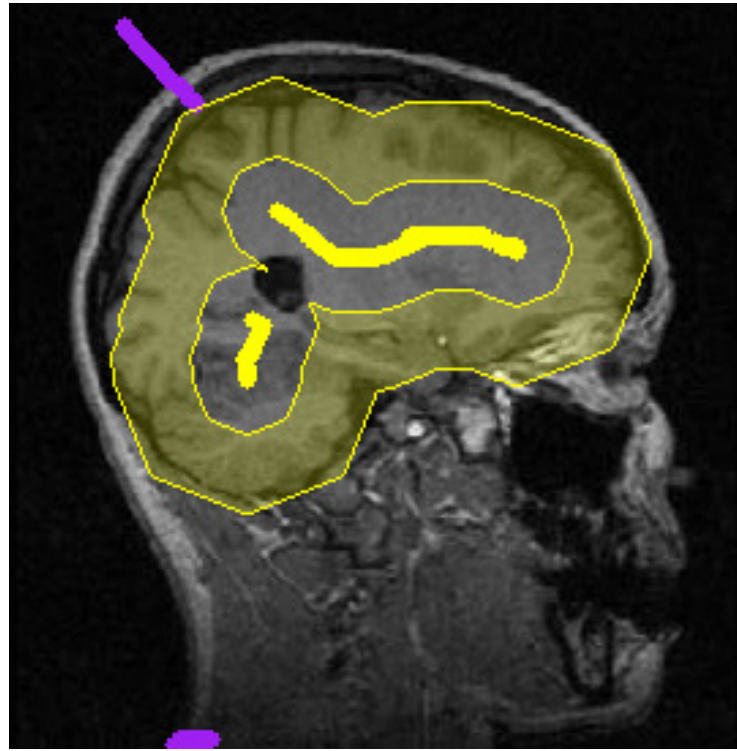

(b)

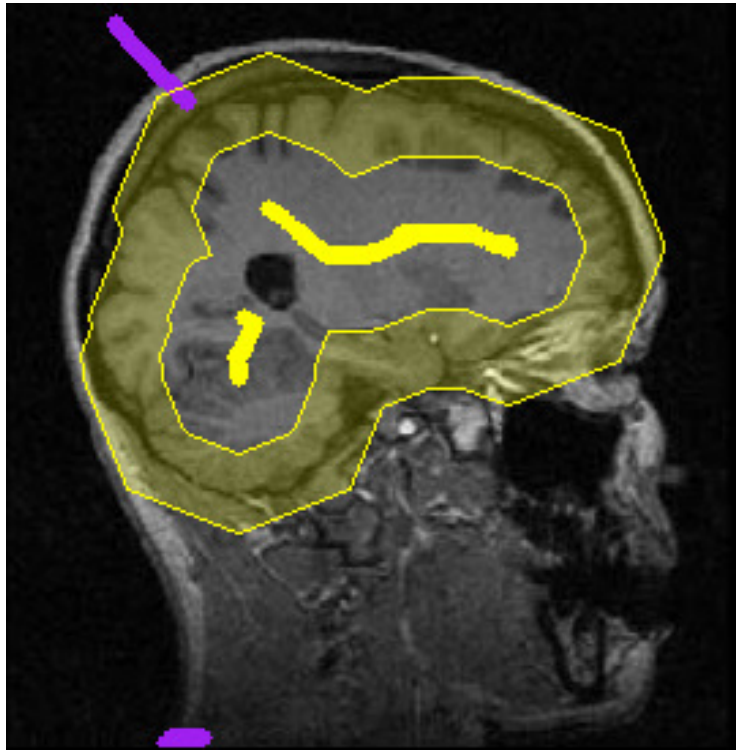

(d)

Figura 6.1: (a-d) A faixa de tamanho fixo da GBC-IFT evolui a partir das sementes, adaptando-se ao conteúdo da imagem. A borda delineada (Figura 1.1d) está restrita dentro da faixa, ajudando a evitar falsas segmentações (Figuras 1.1a-c).

\section{Algoritmo 2. GBC-IFT}

Entrada: $\quad$ Grafo $G=(\mathcal{I}, \mathcal{A})$, conjuntos de sementes $\mathcal{S}_{o}$ e $\mathcal{S}_{b}$, mapa de custos geodésico $C$, floresta geodésica $P$ e $\Delta$.

SAÍDA: $\quad$ mapa de valores dos caminhos $V$ e mapa de rótulos $L$.

Auxiliares: Variável tmp e filas de prioridade $Q, Q_{o}, Q_{b}$ e $Q_{T}$. Cada fila $Q$ deve ter um vetor de status para permitir uma verificação rápida do estado $Q . s t a t u s(s)$ de qualquer pixel $s$, que pode assumir os valores: 0 (pixel $s$ nunca foi inserido na fila $Q$ ), 1 (pixel $s$ está inserido na fila $Q$ ), 2 (pixel $s$ foi removido de $Q)$.

1. Para cada $t \in \mathcal{S}_{o}$ Faça $L(t) \leftarrow 1$.

2. Para cada $t \in \mathcal{S}_{b}$ Faça $L(t) \leftarrow 0$ e insira $t$ em $Q_{b}$.

3. Para cada $t \in \mathcal{I}$ Faça

4. Se $t$ é um nó folha em $P$ então insira $t$ em $Q_{T}$.

5. $\quad F a c ̧ a V(t) \leftarrow f_{w}(\langle t\rangle)$.

6. Se $V(t) \neq+\infty$ então insira $t$ em $Q$.

7. Senão $\operatorname{Se} C(t)-\min _{t \in \mathcal{S}_{b}} C(t)>\Delta$, então 
8. $\quad V(t) \leftarrow 0, L(t) \leftarrow 0$.

9. Insira $t$ nas filas $Q$ e $Q_{b}$.

10. Enquanto $Q \neq \emptyset$, Faça

11. Remova s de $Q$ tal que $V(s)$ é mínimo.

12. Se $L(s)=0$ então

13. Se $s$ está em $Q_{b}$ então remova $s$ de $Q_{b}$.

14. Propaga_bkg(s, $\left.G, Q, Q_{b}, V, L\right)$.

15. Enquanto $Q_{b} \neq \emptyset e \max _{t \in Q_{b}} C(t)-\min _{t \in Q_{b}} C(t)>\Delta$, Faça

16. Remova $t$ de $Q_{b}$ tal que $C(t)$ é máximo.

17. $L(t) \leftarrow 0$ e remova $t$ de $Q$.

18. Propaga_bkg $\left(t, G, Q, Q_{b}, V, L\right)$.

19. Enquanto $\bar{Q}_{T} \neq \emptyset$ e $\max _{t \in Q_{T}} C(t)-\min _{t \in Q_{b}} C(t)>\Delta$, Faça

20. Remova $t$ de $Q_{T}$ tal que $C(t)$ é máximo.

21. Se $Q$.status $(t) \neq 2$, então

22. $\quad L(t) \leftarrow 0$.

23. Se $t$ está em $Q$ então remova $t$ de $Q$.

24. Se $t$ está em $Q_{b}$ então remova $t$ de $Q_{b}$.

25. Propaga_bkg $\left(t, G, Q, Q_{b}, V, L\right)$.

26. Se mudanças foram feitas em $Q_{T}$, então GOTO Linha 15.

27. Senão Se $L(s)=1$ então

28. Insira $s$ em $Q_{o}$.

29. Propaga $(s, G, Q, V, L)$.

30. Enquanto $\max _{t \in Q_{o}} C(t)-\min _{t \in Q_{o}} C(t)>\Delta$, Faça

31. Remova $t$ de $Q_{o}$ tal que $C(t)$ é mínimo.

32. Propaga_obj $\left(t, G, Q, Q_{o}, V, L\right)$.

\section{Algoritmo 3. Propaga}

Entrada: $\quad$ Pixel $s \in \mathcal{I}$, grafo da imagem $G=(\mathcal{I}, \mathcal{A})$, fila de prioridade $Q$, mapa de valores mínimos $V$ e mapa de rótulos $L$.

1. Para cada $t \in \mathcal{A}(s)$ tal que Q.status $(t) \neq 2$, Faça

2. $t m p \leftarrow f_{w}\left(\pi_{s} \cdot\langle s, t\rangle\right)$.

3. Se tmp $<V(t)$, então

4. Se $t \in Q$, então remova $t$ de $Q$.

5. $\quad$ Faça $V(t) \leftarrow t m p, L(t) \leftarrow L(s)$.

6. Insira $t$ em $Q$.

\section{Algoritmo 4. Propaga_BKG}

Entrada: $\quad$ Pixel $s \in \mathcal{I}$, grafo da imagem $G=(\mathcal{I}, \mathcal{A})$, filas de prioridade $Q$ e $Q_{b}$, mapa de valores mínimos $V$ e mapa de rótulos $L$.

1. Propaga $(s, G, Q, V, L)$.

2. Para cada $t \in \mathcal{A}(s)$, Faça

3. $\quad$ Se $Q_{b}$. status $(t)=0$, então

4. $\quad$ Se $L(t) \neq 0$ ou Q.status $(t) \neq 2$, então

5. Insira t em $Q_{b}$.

Algoritmo 5. Propaga_oBJ

Entrada: Pixel $s \in \mathcal{I}$, grafo da imagem $G=(\mathcal{I}, \mathcal{A})$, filas de prioridade $Q$ e $Q_{o}$, mapa de valores mínimos $V$ e mapa de rótulos $L$.

1. Para cada $t \in \mathcal{A}(s)$, Faça

2. Se $Q_{o} . \operatorname{status}(t)=0$, então

3. $\quad L(t) \leftarrow 1$.

4. Removat de $Q$.

5. Insira $t$ em $Q_{o}$.

6. Propaga $(t, G, Q, V, L)$. 


\subsubsection{Corretude do Algoritmo GBC-IFT}

A cada passo o algoritmo considera dois objetos candidatos: $\mathcal{O}_{o}=\{t \in \mathcal{I} \mid L(t)=1 \wedge$ Q.status $(t)=2\}$ obtido pelo crescimento da região das sementes internas e $\mathcal{O}_{b}=\{t \in \mathcal{I} \mid L(t) \neq 0 \vee Q$.status $(t) \neq 2\}$ que é composto pelo complemento dos pixels que foram marcados definitivamente como pertencentes ao plano de fundo ${ }^{1}$, como podemos ver na Figura 6.2. As filas de prioridade $Q_{o}$ e $Q_{b}$ são usadas para armazenar pixels em $\mathcal{B}\left(\mathcal{O}_{o}\right)$ e $\mathcal{B}\left(\mathcal{O}_{b}\right)$ respectivamente. O algoritmo apresenta os seguintes invariantes:

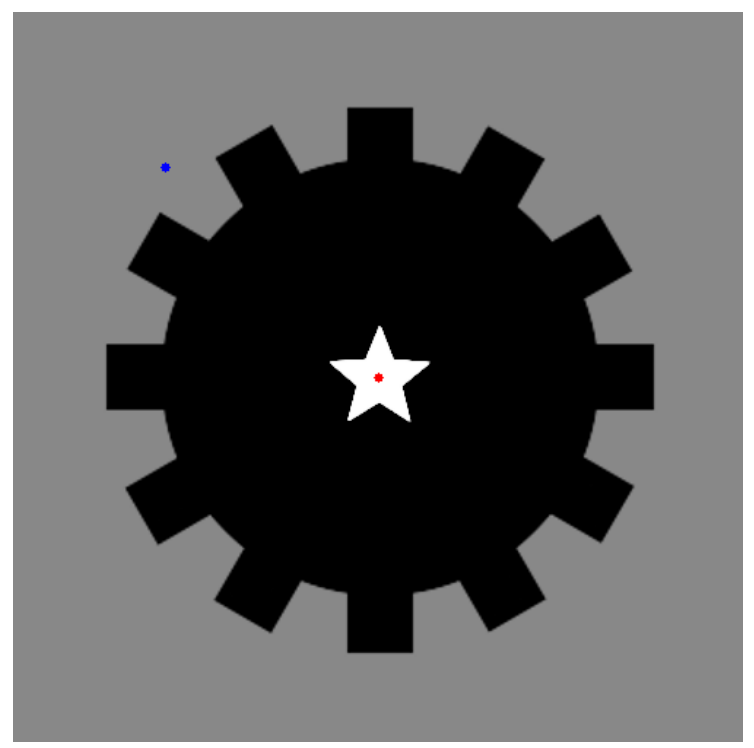

(a) Imagem original com sementes

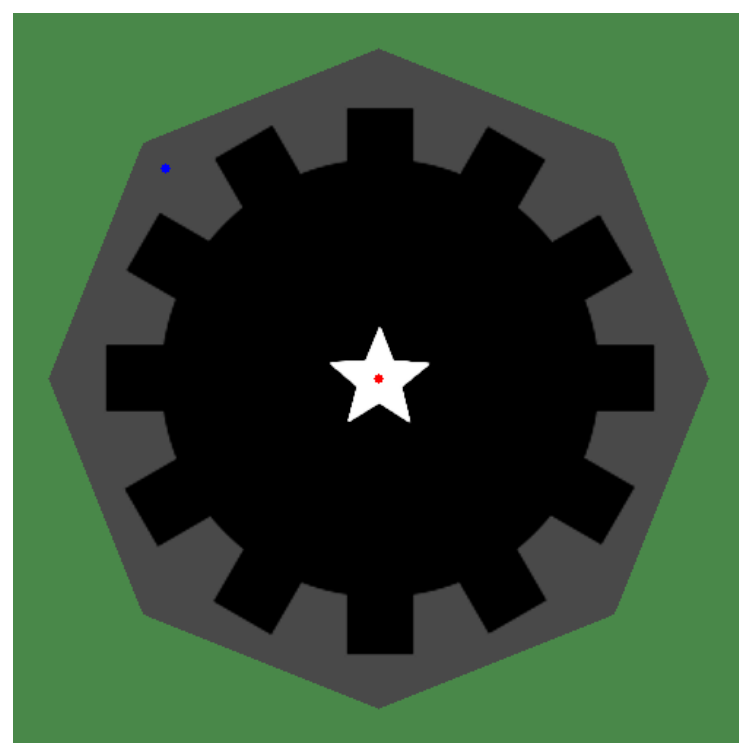

(b) Área verde delimitada como fundo, para $\Delta=25$ pixels

Figura 6.2: Exemplo de área delimitada como fundo. Temos as sementes de fundo em azul e as sementes de objeto em vermelho. A área verde em (b) foi delimitada logo no início do algoritmo como sendo pertencente ao fundo.

Lema 1. Durante a execução do Algoritmo 2, temos que: $\min _{t \in \mathcal{B}\left(\mathcal{O}_{o}\right)} C(t) \leq \min _{t \in \mathcal{B}\left(\mathcal{O}_{b}\right)} C(t)$.

Demonstração. Seja $s \in \mathcal{B}\left(\mathcal{O}_{b}\right)$ um pixel tal que $C(s)=\min _{t \in \mathcal{B}\left(\mathcal{O}_{b}\right)} C(t)$. Voltando atrás no mapa de predecessores $P$ a partir de $s$, sempre chegaremos em um pixel $t \in \mathcal{B}\left(\mathcal{O}_{o}\right)$ tal que $C(t) \leq C(s)$, uma vez que o custo $C$ é uma função não decrescente ao longo dos caminhos da floresta $P$ e suas raízes sempre pertencem a $\mathcal{O}_{o}$. Note que por definição temos que $\mathcal{O}_{o} \subseteq \mathcal{O}_{b}{ }^{2}$.

Proposição 1. Ao término de cada iteração do laço principal (Linha 10) do Algoritmo 2, são invariantes do algoritmo que ambos $\mathcal{O}_{o}$ e $\mathcal{O}_{b}$ satisfazem a $G B C$.

Demonstração. Provando por indução, temos:

Base: No início o objeto $\mathcal{O}_{o}$ será dado pelas sementes internas, isto é, $\mathcal{O}_{o}=\mathcal{S}_{o}$. Como os custos geodésicos nas sementes são 0, $\mathcal{O}_{o}$ claramente satisfaz a GBC. As Linhas (3-9) garantem a construção de um primeiro objeto $\mathcal{O}_{b}$ que satisfaz a $\mathrm{GBC}$, pois todo pixel $t$ que viola a restrição (Linha 7 ) tem seu rótulo atribuído como 0 (Linha 8).

Passo da indução: Supondo que após $n-1$ iterações, vale que $\mathcal{O}_{o}$ e $\mathcal{O}_{b}$ satisfazem a GBC, vamos mostrar que após $n$ iterações, elas ainda continuam valendo. Para isso precisamos analisar dois casos:

Caso 1 - quando $\mathcal{O}_{o}$ é alterada: qualquer adição de um pixel $s$ em $\mathcal{O}_{o}$ (Linha 11) resultará na execução das Linhas (27-32). Este trecho restabelece a GBC para $\mathcal{O}_{o}$, aumentando $\min _{r \in \mathcal{B}\left(\mathcal{O}_{o}\right)} C(r)$ por meio de sucessivas remoções de pixels $t$ da fila $Q_{o}$ (Linha 31 ), que já não podem mais estar em $\mathcal{B}\left(\mathcal{O}_{o}\right)$. Assim sendo, todos os vizinhos de $t$ devem ser atribuídos para $\mathcal{O}_{o}$, o que é feito pela chamada do Algoritmo 5 (Linha 32), resultando na expansão de $\mathcal{O}_{o}$.

Note que as alterações feitas pela função corretiva acima não serão desfeitas em passos futuros uma vez que a atribuição de $s$ para $\mathcal{O}_{o}$ necessita que $C(s) \leqslant \min _{r \in \mathcal{B}\left(\mathcal{O}_{o}\right)} C(r)+\Delta$.

\footnotetext{
${ }^{1}$ Note que pelas leis de De Morgan, temos que $\mathcal{O}_{b}=\{t \in \mathcal{I} \mid \operatorname{not}(L(t)=0 \wedge$ Q.status $(t)=2)\}$

${ }^{2} \mathcal{O}_{o} \subseteq\{t \in \mathcal{I} \mid L(t) \neq 0\} \subseteq \mathcal{O}_{b}$
} 
As operações para restabelecer as GBC para $\mathcal{O}_{o}$ resultam na aquisição de mais pixels para $\mathcal{O}_{o}$. Sejam $\mathcal{O}_{x}^{\triangleleft}$ e $\mathcal{O}_{x}^{\triangleright}$ o conjunto $\mathcal{O}_{x}$ antes e depois das aquisições mencionadas acima (com $x$ sendo $o$ ou $b$ ). Para

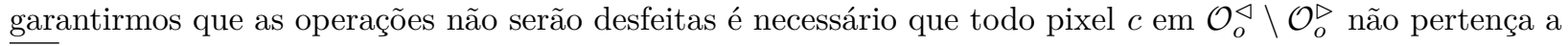
$\overline{\mathcal{O}_{b}^{\triangleleft}}=\mathcal{I} \backslash \mathcal{O}_{b}^{\triangleleft}$

Vamos provar isso por contradição. Seja um pixel $t$ que pertença a $\mathcal{B}\left(\mathcal{O}_{o}^{\triangleleft}\right)$ e $\mathcal{B}\left(\mathcal{O}_{b}^{\triangleleft}\right)$ e deve ser removido de $\mathcal{B}\left(\mathcal{O}_{o}^{\triangleleft}\right)$ (Linha 31 ) pois existe um pixel $v$ que viola a $\mathrm{GBC}$ para $\mathcal{O}_{o}^{\triangleleft}$, isto é, $C(v)>\min _{s \in \mathcal{B}\left(\mathcal{O}_{o}^{\triangleleft}\right)} C(s)+\Delta$.

Pelo algoritmo, os vizinhos de $t$ são atribuídos para $\mathcal{B}\left(\mathcal{O}_{o}^{\triangleright}\right)$, no entanto pelo menos um destes vizinhos pertence à $\mathcal{O}_{b}^{\triangleleft}$ uma vez que $t$ pertence a $\mathcal{B}\left(\mathcal{O}_{b}^{\triangleleft}\right)$. Com isso e usando o Lema 1 , podemos escrever que:

$$
C(t)=\min _{s \in \mathcal{B}\left(\mathcal{O}_{o}^{\triangleleft}\right)} C(s) \leqslant \min _{s \in \mathcal{B}\left(\mathcal{O}_{b}^{\triangleleft}\right)} C(s)
$$

Porém como $t$ pertence a $\mathcal{B}\left(\mathcal{O}_{b}^{\triangleleft}\right)$, temos:

$$
C(t)=\min _{s \in \mathcal{B}\left(\mathcal{O}_{o}^{\triangleleft}\right)} C(s)=\min _{s \in \mathcal{B}\left(\mathcal{O}_{b}^{\triangleleft}\right)} C(s)
$$

Sabendo que a GBC é válida para $\mathcal{O}_{b}^{\triangleleft}$ e combinando com a Equação (6.2.2), temos que para todo $q \in \mathcal{O}_{b}^{\triangleleft}$ vale:

$$
C(q) \leqslant \min _{s \in \mathcal{B}\left(\mathcal{O}_{b}^{\triangleleft}\right)} C(s)+\Delta=\min _{s \in \mathcal{B}\left(\mathcal{O}_{o}^{\triangleleft}\right)} C(s)+\Delta
$$

Como $v$ pertence a $\mathcal{O}_{b}^{\triangleleft}$, podemos concluir que $C(v) \leqslant \min _{s \in \mathcal{B}\left(\mathcal{O}_{o}^{\triangleleft}\right)} C(s)+\Delta$, o que é uma contradição com a premissa de que $v$ viola a GBC para $\mathcal{O}_{o}$.

Logo, podemos concluir que o algoritmo está correto quando $\mathcal{O}_{o}$ é alterado.

Caso 2 - quando $\mathcal{O}_{b}$ é alterada: Similarmente, a remoção de um pixel $s$ de $\mathcal{O}_{b}$ (Linha 11) resultará na execução das Linhas (12-26). A chamada do Algoritmo 4 (Linha 14) avalia os vizinhos de $s$ para atualizar $Q_{b}$, que deverá conter os nós de $\mathcal{B}\left(\mathcal{O}_{b}\right)$. Se o valor $\min _{r \in \mathcal{B}\left(\mathcal{O}_{b}\right)} C(r)$ diminuir, então os pixels $t$ com $C(t)>$ $\min _{r \in \mathcal{B}\left(\mathcal{O}_{b}\right)} C(r)+\Delta$ serão sucessivamente removidos de $\mathcal{O}_{b}$ até que a GBC esteja satisfeita para $\mathcal{O}_{b}$, gerando um encolhimento de $\mathcal{O}_{b}$. Para uma implementação mais eficiente, testamos apenas os nós folha em $Q_{T}$ (Linhas 19-25) e os pixels em $Q_{b}$ (Linhas 15-18).

Estas operações de restabelecimento da GBC para $\mathcal{O}_{b}$ resultam em remoção de um subconjunto de pixels de $\mathcal{O}_{b}$. Para garantirmos a corretude destas operações, precisamos que todo pixel $c$ em $\mathcal{O}_{b}^{\triangleleft} \backslash \mathcal{O}_{b}^{\triangleright}$ não pertença a $\mathcal{O}_{o}^{\triangleleft}$, para evitar conflito com a GBC para $\mathcal{O}_{o}$.

Novamente, provando por contradição, seja um pixel $t$ que pertença a $\mathcal{O}_{o}^{\triangleleft}$ e $\mathcal{O}_{b}^{\triangleleft} \backslash \mathcal{O}_{b}^{\triangleright}$, que esteja violando a GBC para $\mathcal{O}_{b}^{\triangleleft}, \operatorname{logo:}$

$$
C(t)>\min _{s \in \mathcal{B}\left(\mathcal{O}_{b}^{\triangleleft}\right)} C(s)+\Delta
$$

Porém como $t$ pertence a $\mathcal{O}_{o}^{\triangleleft}$, temos:

$$
C(t) \leqslant \min _{s \in \mathcal{B}\left(\mathcal{O}_{o}^{\triangleleft}\right)} C(s)+\Delta
$$

Combinando a Equação (6.2.5) com o Lema 1, temos:

$$
C(t) \leqslant \min _{s \in \mathcal{B}\left(\mathcal{O}_{o}^{\triangleleft}\right)} C(s)+\Delta \leqslant \min _{s \in \mathcal{B}\left(\mathcal{O}_{b}^{\triangleleft}\right)} C(s)+\Delta
$$
$\mathcal{O}_{b}$.

O que gera uma contradição com a Eq. (6.2.4). Logo, temos que o algoritmo está correto quando alteramos

Portanto, podemos ver que qualquer alteração feita pelo algoritmo em $\mathcal{O}_{o}$ ou $\mathcal{O}_{b}$ mantém a GBC válida para os dois conjuntos.

Lema 2. Durante a execução do Algoritmo 2, $\min _{t \in \mathcal{B}\left(\mathcal{O}_{b}\right)} C(t)$ nunca aumenta seu valor.

Demonstração. Todo pixel $s$ tal que $C(s)=\min _{t \in \mathcal{B}\left(\mathcal{O}_{b}\right)} C(t)$, possui um predecessor $P(s)$ com $C(P(s)) \leq C(s)$, uma vez que o mapa de custos $C$ é uma função não-decrescente nos caminhos da floresta $P$. O valor de 
$\min _{t \in \mathcal{B}\left(\mathcal{O}_{b}\right)} C(t)$ pode apenas aumentar se removermos de $\mathcal{B}\left(\mathcal{O}_{b}\right)$ seus pixels com custo mínimo ${ }^{3}$.

Porém neste caso $P(s)$ seria inserido em $\mathcal{B}\left(\mathcal{O}_{b}\right)$, não aumentando o valor, ou isso significaria que $P(s) \notin$ $\mathcal{O}_{b}$. Mas, neste caso, teríamos que algum antecessor de $P(s)$ está em $\mathcal{B}\left(\mathcal{O}_{b}\right)$, de modo que o valor não pode aumentar. Note que as raízes da floresta $P$ são as sementes internas e $S_{o} \subset \mathcal{O}_{b}$.

A cada iteração do laço principal, o algoritmo GBC-IFT sempre modifica a borda candidata a objeto $\left(\mathcal{O}_{o}\right.$ ou $\left.\mathcal{O}_{b}\right)$ que possuir a maior energia $\varepsilon_{\infty}$, por meio do arco com pior custo em sua borda (Linha 11). Este é um evento que não deverá ser desfeito, pois é a única mudança de um pixel que pode melhorar o candidato a objeto com pior energia $\varepsilon_{\infty}$. Mudar outros pixels não vai melhorá-la. O Lema 2 nos garante que mudanças corretivas feitas para restabelecer a GBC não poderão ser desfeitas. No final, todos os pixels terão sido removidos da fila $Q$ e os objetos $\mathcal{O}_{o}$ e $\mathcal{O}_{b}$ terão convergido para o mesmo conjunto de pixels que satisfaz a GBC (Proposição 1). Portanto, temos um mínimo global da função de energia $\varepsilon_{\infty}$ sujeita à GBC.

\subsection{Análise do parâmetro $\Delta$}

Uma dúvida natural quando analisamos o método é descobrir qual seria o parâmetro $\Delta$ adequado. Se ele for muito pequeno, corremos o risco de restringir demais a borda do objeto, fazendo com que a segmentação resultante fique com um erro muito grande; por outro lado, um $\Delta$ grande demais faz o método degenerar para uma IFT sem restrição alguma, fazendo o método ser inócuo.

Atacamos esta problema gerando curvas de energia máxima (valor de $\varepsilon_{\infty}$ ) e média (valor médio de $\bar{\omega}$ na borda encontrada) da segmentação encontrada para valores $\Delta$ que vão até o método convergir para a IFT tradicional. Com isso podemos analisar para cada imagem se existem segmentações estáveis, isto é, segmentações que resistem a alterações de $\Delta$, que são verificadas nos gráficos por patamares estáveis de energia nas duas curvas simultaneamente. Uma escolha possível é tomar a segmentação que seja mais robusta a variações de $\Delta$. As Figuras 6.3 e 6.4 ilustram alguns exemplos.

\footnotetext{
${ }^{3}$ Devido ao crescimento de região a partir das sementes externas em $\mathcal{S}_{b}$, temos que a cardinalidade $\left|\overline{\mathcal{O}_{b}}\right|$ de $\overline{\mathcal{O}_{b}}=\mathcal{I} \backslash \mathcal{O}_{b}$ nunca diminui e a cardinalidade $\left|\mathcal{O}_{b}\right|$ de $\mathcal{O}_{b}$ nunca aumenta. Logo, o único modo de remover $s$ de $\mathcal{B}\left(\mathcal{O}_{b}\right)$ é removendo-o de $\mathcal{O}_{b}$
} 
Energia de segmentação

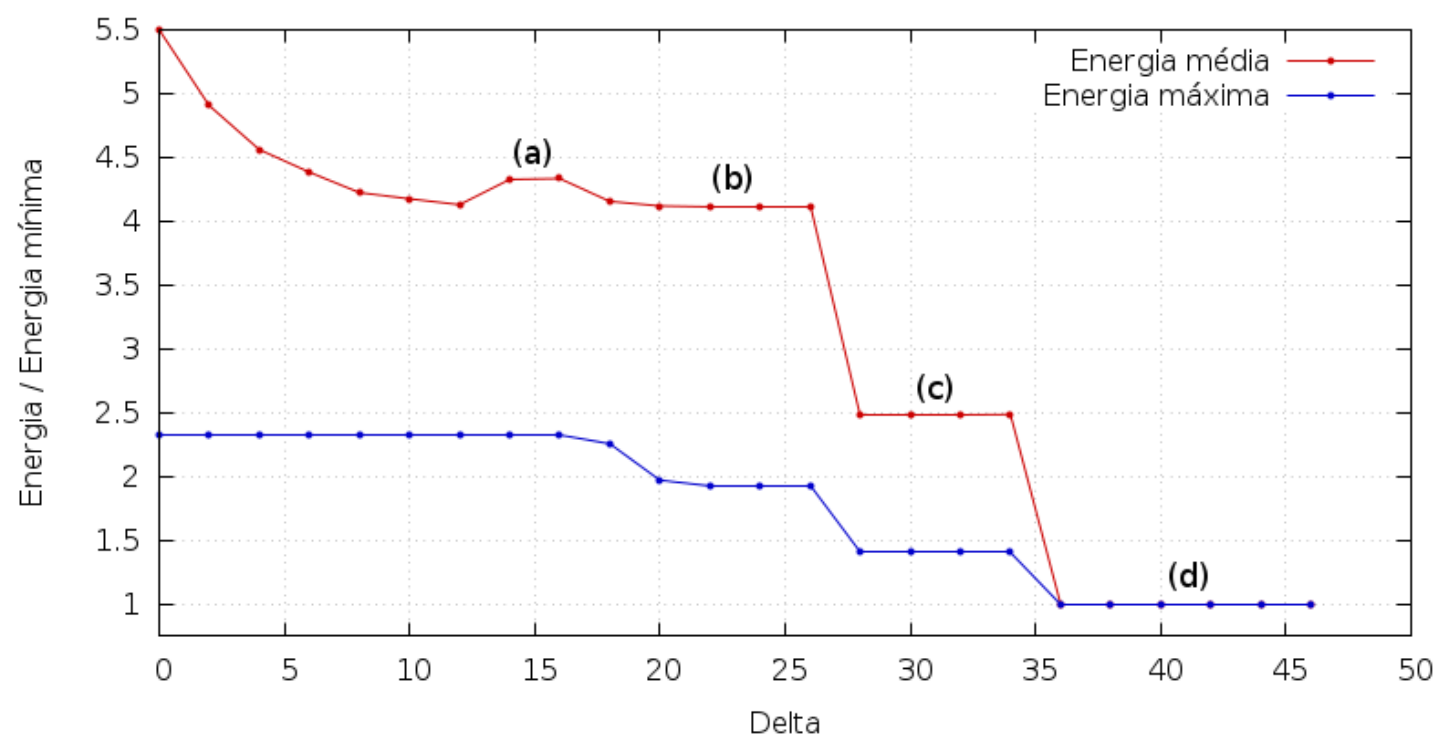

Grafico de energias por $\Delta$. Em vermelho a energia média da borda e em azul a energia máxima.

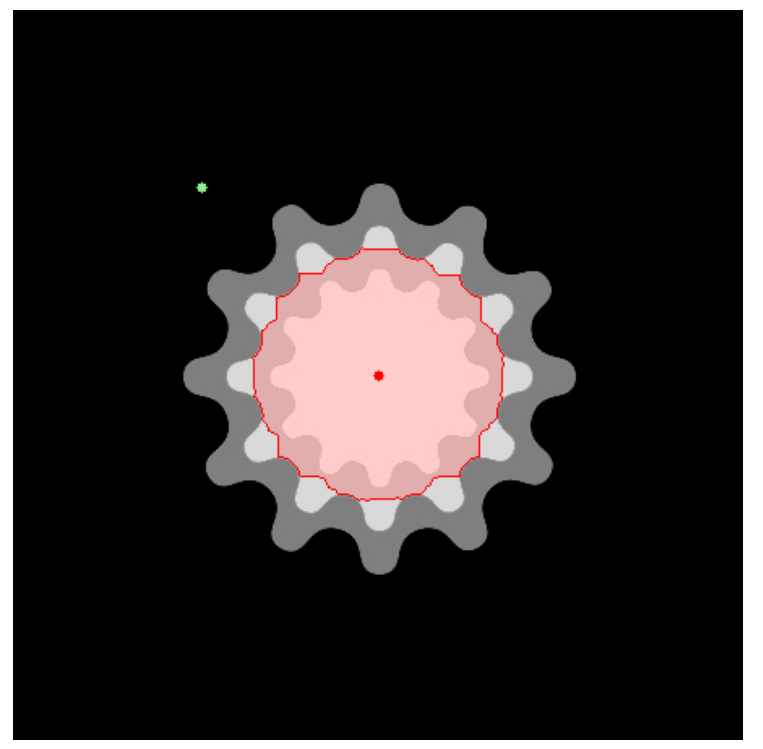

(a)

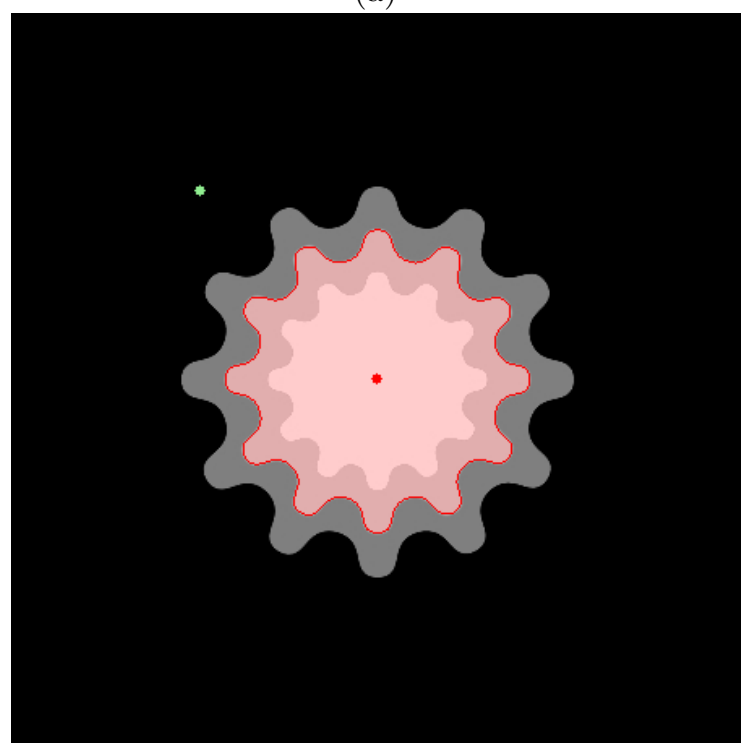

(c)

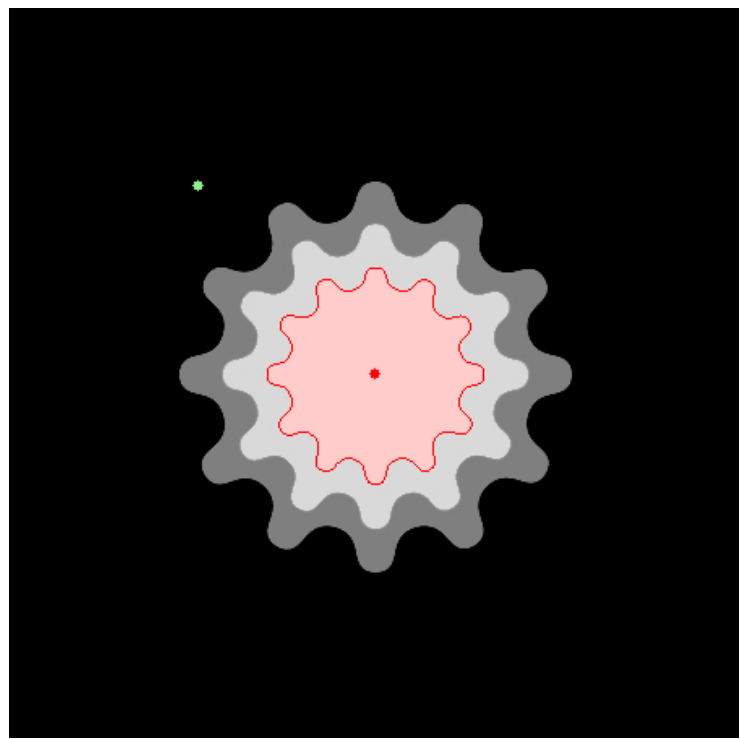

(b)

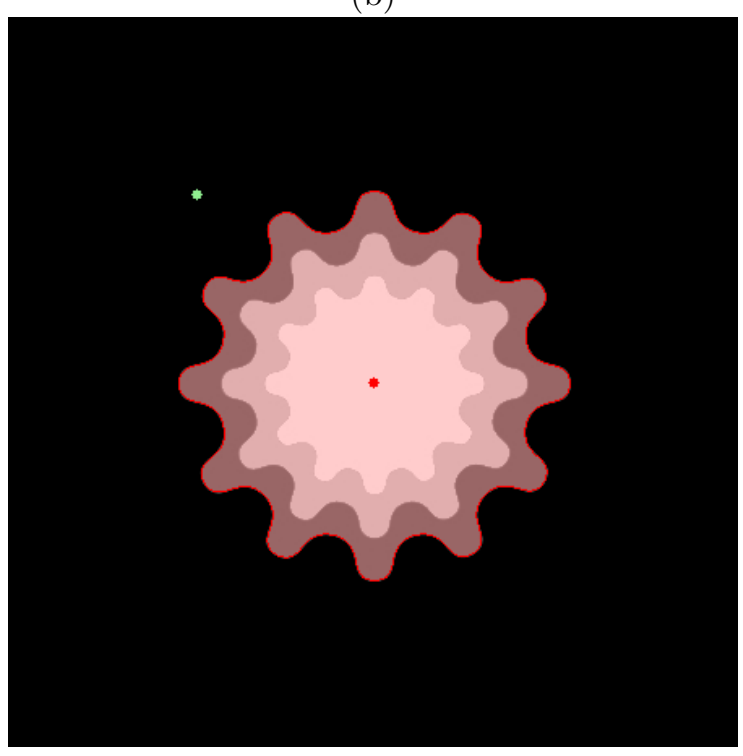

(d) 
Energia de segmentação

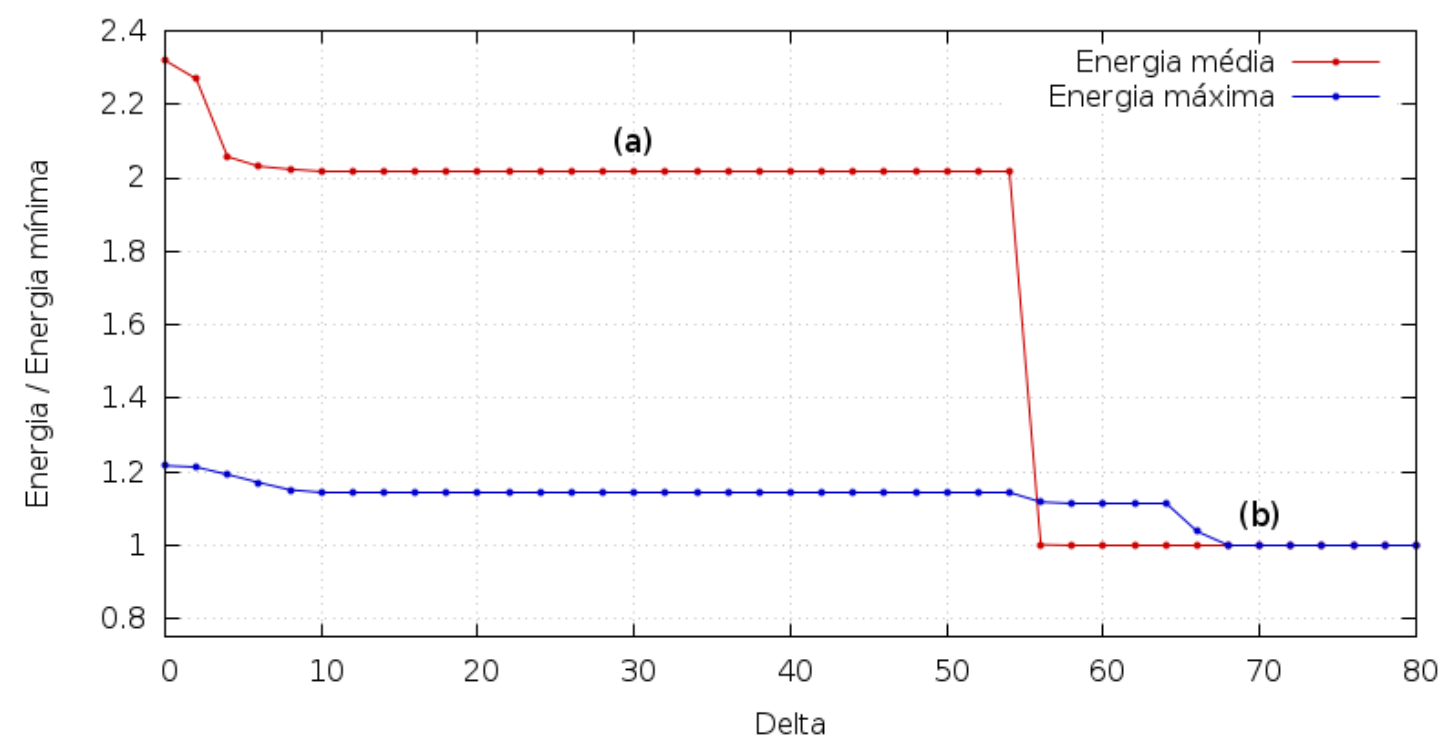

Grafico de energias por $\Delta$

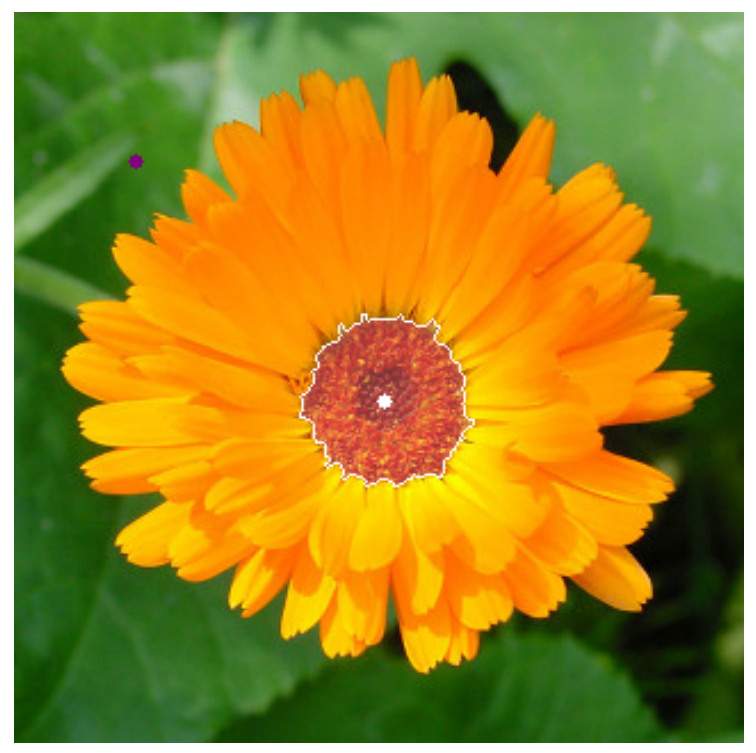

(a)

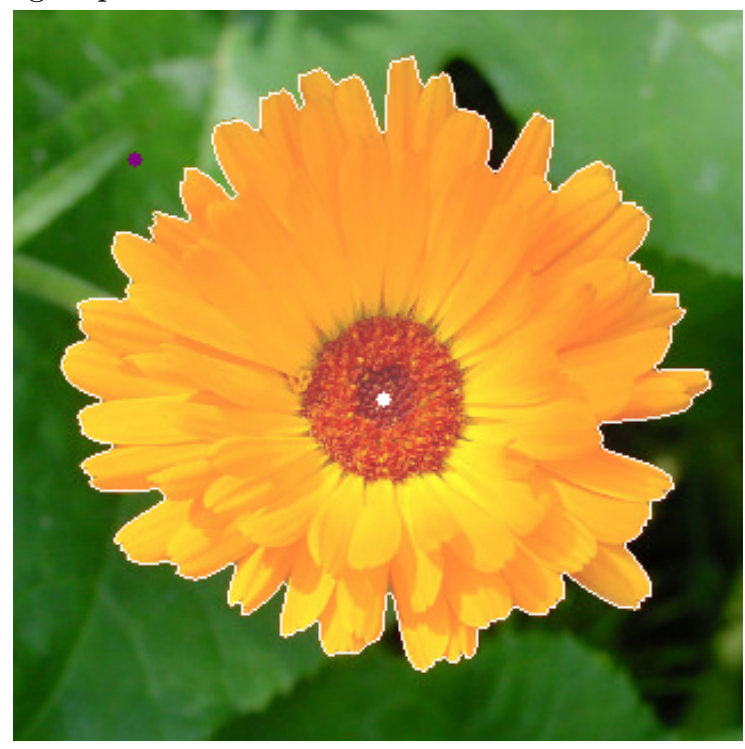

(b)

Figura 6.4: Comparação entre segmentações usando valores de $\Delta$ nos quais há estabilidade na segmentação.

\subsection{Combinação com outras restrições}

Como a GBC é uma restrição que na prática apenas restringe uma faixa de ação para um método específico, podemos combina-la com outras restrições de modo que elas atuem somente nesta faixa. Para isso, podemos modificar o grafo da imagem e a função de custo, de forma análoga à feita em [MM13b], de modo a obter também os benefícios de polaridade de borda como na OIFT e da restrição de forma GSC, resultando em um método que chamamos de GBC-GSC-OIFT, que nos dá ainda mais poder para controlar aspectos da segmentação, nos dando um método bastante versátil.

As figuras 6.5 e 6.6 ilustram este método, com uma comparação de acurácia média, medida pelo coeficiente de Dice, assumindo diferentes conjuntos de sementes obtidos pela erosão e dilatação do gabarito. As sementes externas foram obtidas assimetricamente usando um raio maior na dilatação (o dobro do raio das sementes internas) para gerar uma situação mais desafiadora. O gabarito foi obtido de um especialista em radiologia da Universidade da Pennsylvania.

Pudemos notar uma melhora considerável para os métodos propostos (GBC-IFT e GBC-GSC-OIFT (com $\Delta$ sendo o raio de erosão)), em relação aos métodos anteriores (IRFC, GSC-IFT e GSC-OIFT). 

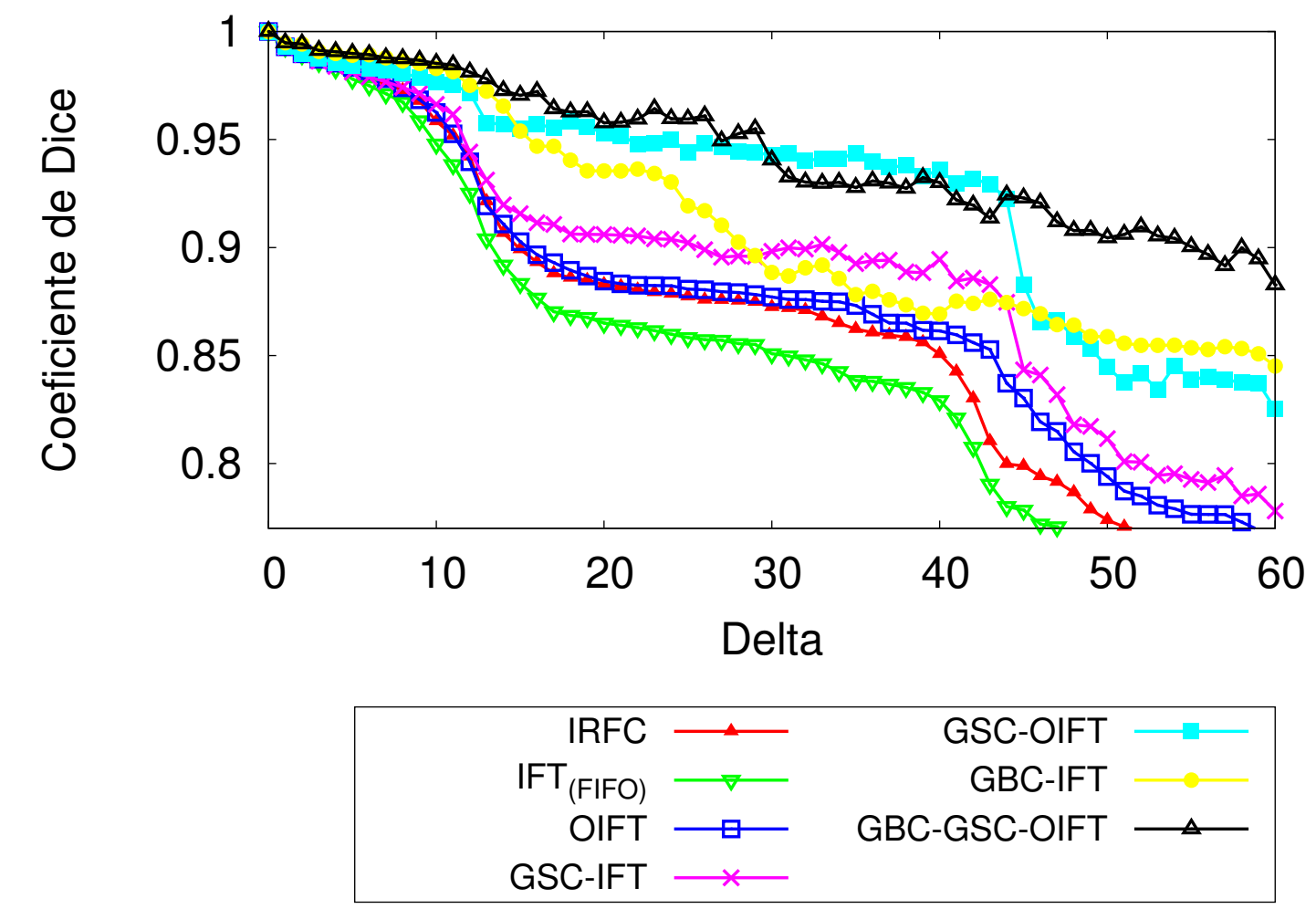

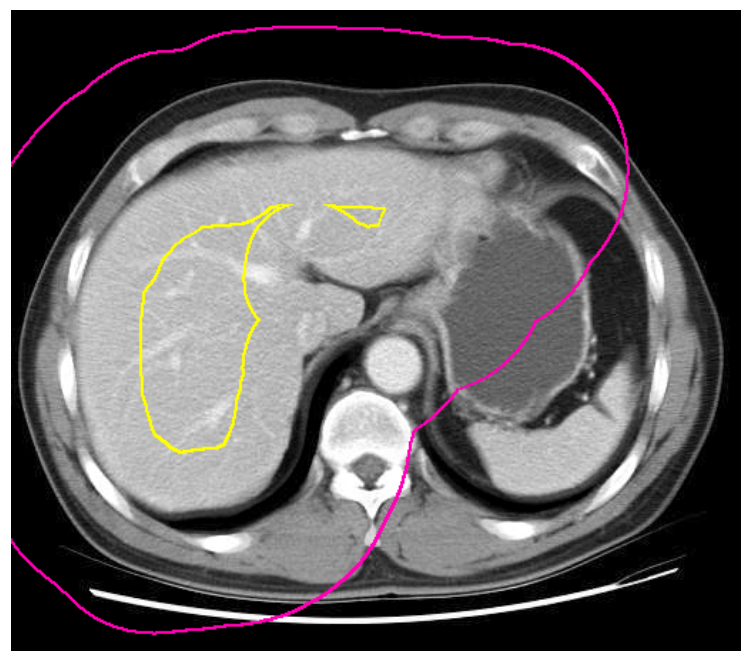

(a) Sementes

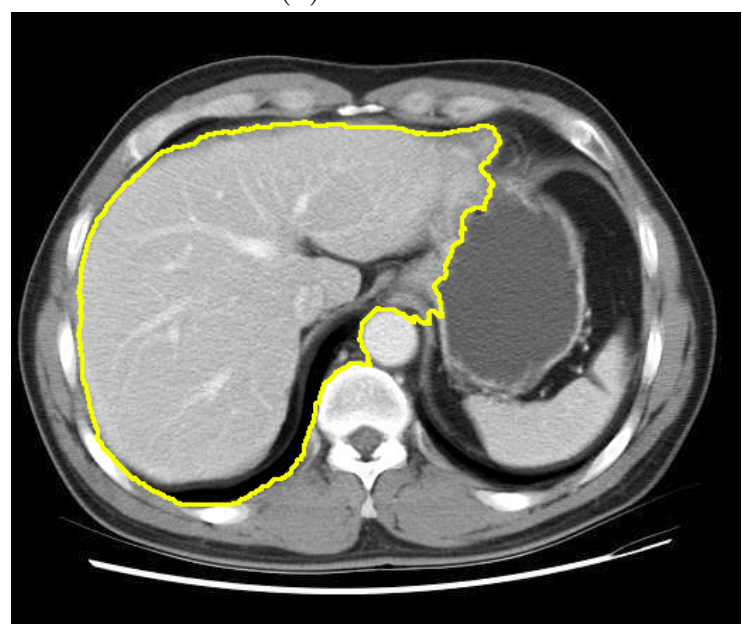

(c) GSC-OIFT

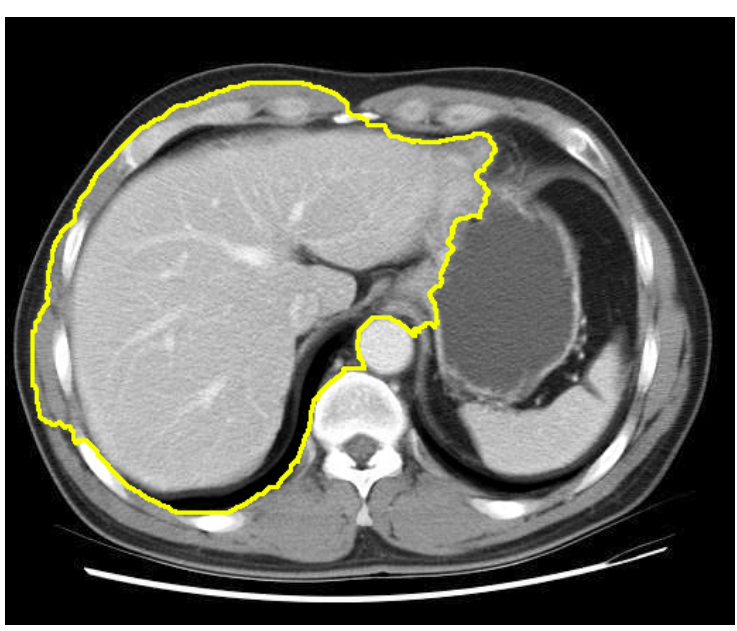

(b) GSC-IFT

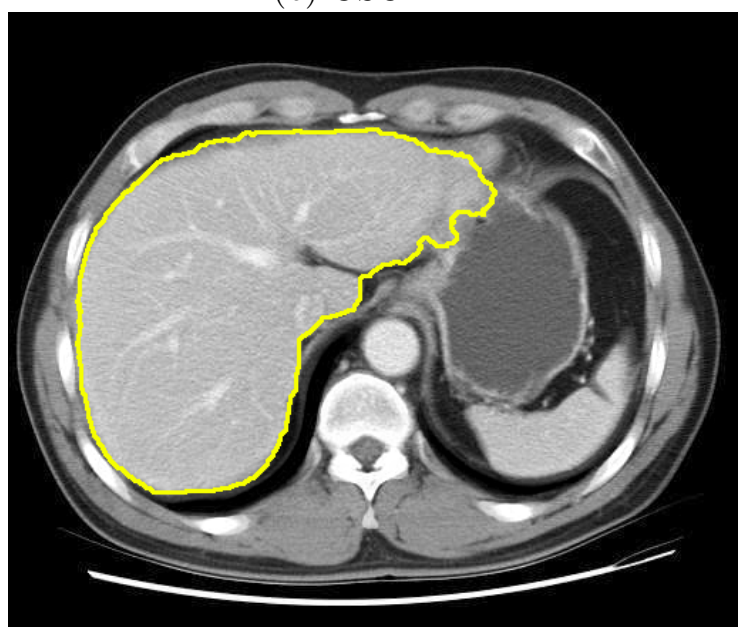

(d) GBC-GSC-OIFT

Figura 6.5: Curva comparativa de acurácia entre diversos métodos realizando segmentações do fígado em uma imagem de tomografia computadorizada. Nas imagens, ilustramos para um dos conjuntos de sementes. 


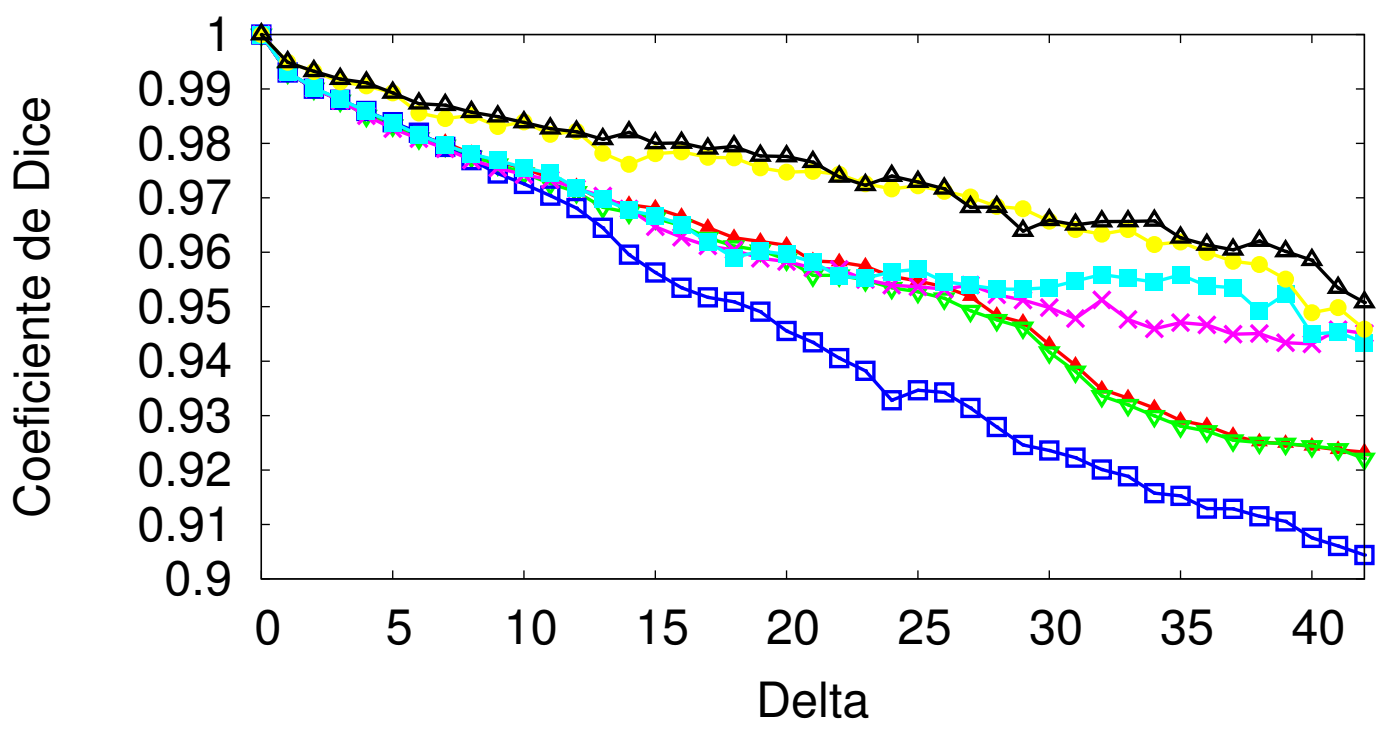

$\begin{array}{rr}\text { IRFC } \longleftarrow & \text { GSC-OIFT } \\ \text { IFT } & \text { GBC-IFT } \\ \text { OIFT } \longleftarrow & \text { GBC-GSC-OIFT } \longleftarrow \\ \text { GSC-IFT } \longrightarrow x & \end{array}$

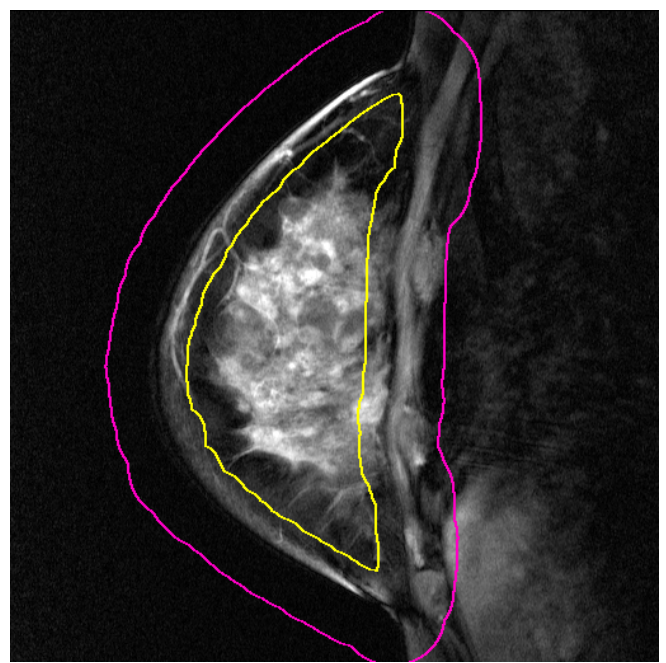

(a) Sementes

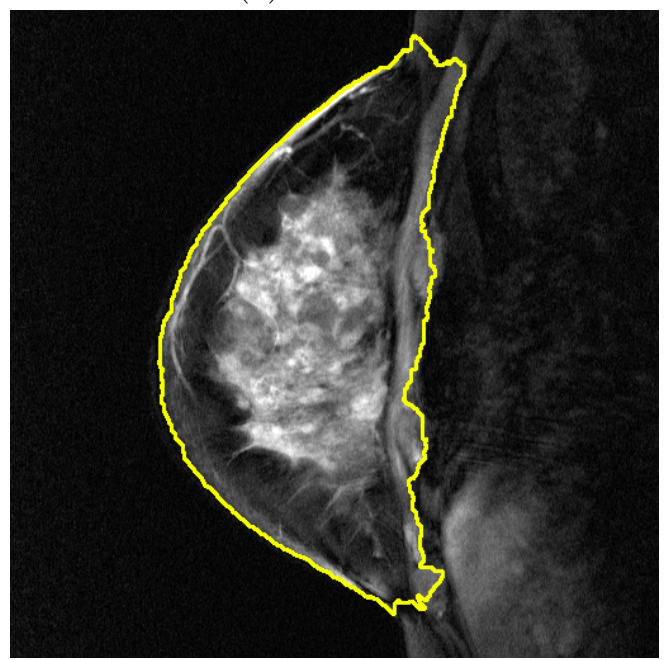

(c) GSC-OIFT

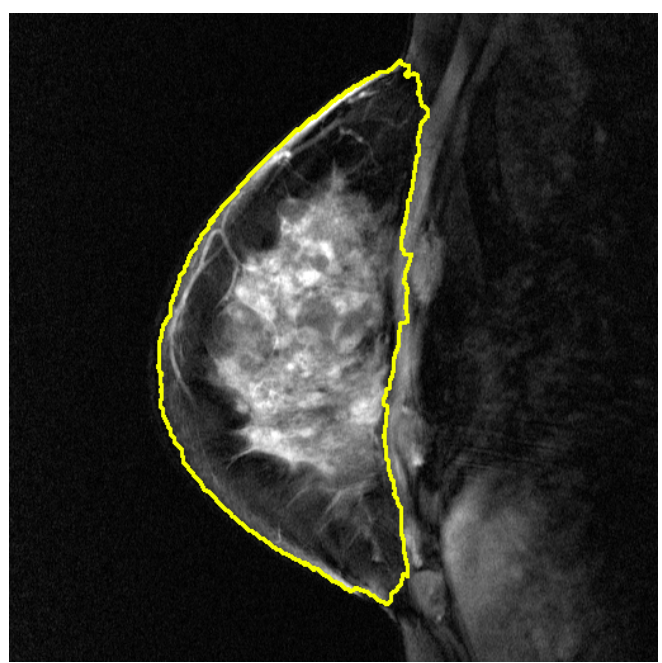

(b) GSC-IFT

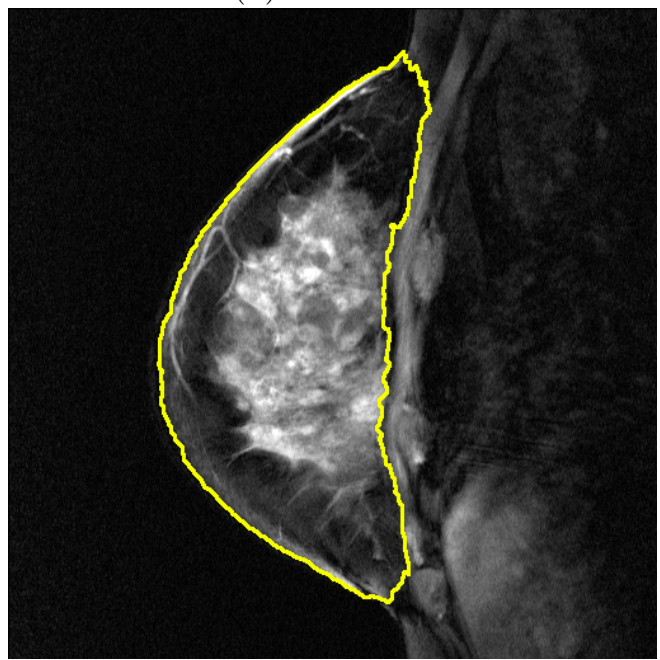

(d) GBC-GSC-OIFT

Figura 6.6: Curva comparativa de acurácia entre diversos métodos realizando segmentações do seio em uma imagem de tomografia computadorizada. Nas imagens, ilustramos para um dos conjuntos de sementes. 


\section{Capítulo 7}

\section{Faixa de Restrição Geodésica Relativa}

Agora que apresentamos a GBC, podemos mostrar uma evolução que consiste em usar uma parâmetro relativo no lugar de um fixo para controlar a segmentação. Em alguns casos, o parâmetro absoluto da GBC-IFT nos impede de contornar variações que são relativamente pequenas que são grandes em termos absolutos. Mostraremos que os resultados anteriores mantiveram-se consistentes e ainda ficaram mais robustos a variações de escala dos objetos e das imagens a serem processadas.

\subsection{Faixa de Restrição Relativa}

No método original, o parâmetro $\Delta$ é um valor absoluto fixo. Isso pode tanto nos ajudar a regularizar formas, como vimos no capítulo anterior mas pode também impedir que algumas formas sejam segmentadas. Podemos ver um exemplo disso na Figura 7.1, onde existe uma forma que possui uma variação relativamente grande comparada ao tamanho total da forma, porém pequena em termos absolutos, de forma que este objeto é segmentado no lugar de um outro que é mais interessante por ter uma variação relativa menor, ou seja, menos irregular, porém tem uma variação grande em termos absolutos, violando assim a GBC e impedindo a forma de ser segmentada.

O fato do parâmetro $\Delta$ ser um valor absoluto, faz com que objetos de escalas distintas sejam comparados sob a mesma óptica, podendo resultar em segmentações distintas até mesmo de objetos similares com tamanhos diferentes na mesma imagem, deixando assim a restrição não robusta a escala.

Um modo que encontramos para contornar este efeito foi especificar um parâmetro $p$ no lugar de $\Delta$, como sendo um valor relativo que deve ser aplicado sobre o valor mínimo, de modo que o seu efeito seja relativo à escala encontrada no processo até dado instante. Com isso, podemos enunciar uma nova definição:

Definição 2 (Faixa de Restrição Geodésica Relativa - Relative Geodesic Band Constraint - RGBC). Um objeto $\mathcal{O}$ satisfaz a faixa de restrição geodésica de proporção p se $\forall t \in \mathcal{O}$ vale que $C(t) \leq \min _{s \in \mathcal{B}(\mathcal{O})} C(s) \cdot(1+p)$.

Essencialmente, a restrição foi alterada trocando a soma de uma constante, no caso $\Delta$, por uma multiplicação por uma constante, de modo que agora o tamanho da faixa de restrição passa a ser alterado durante o processo de forma relativa ao valor mínimo parcial de um dado instante. Isso nos permite segmentar objetos que antes eram ignorados pelo processo de segmentação.

\subsection{Algoritmo}

O algoritmo que implementa a RGBC-IFT é essencialmente o mesmo da GBC-IFT, apenas com a alteração das comparações com $\Delta$ que ocorrem nas linhas 7, 15, 19 e 30 do Algoritmo 2 de forma a atender a RGBC e não a GBC, isto é, no lugar de comparar se um valor é maior que $\Delta$, comparamos se este valor é maior que $p$ vezes o valor mínimo que estiver sendo considerado no momento. Os Algortimos 3, 4, 5 não sofreram alterações. A corretude deste método usando a RGBC é provada de forma análoga à prova feita no Capítulo 6 referente à GBC, pois a mudança matemática da restrição não afeta os invariantes do método.

\subsection{Modelos pré-definidos de mapas de custos}

Assim como no método original, podemos gerar o mapa de custos de outras maneiras, isto inclui usar outras funções de custo na primeira etapa ou utilizar mapas de custo pré-definidos, obtidos externamente. A 
Energia de segmentação

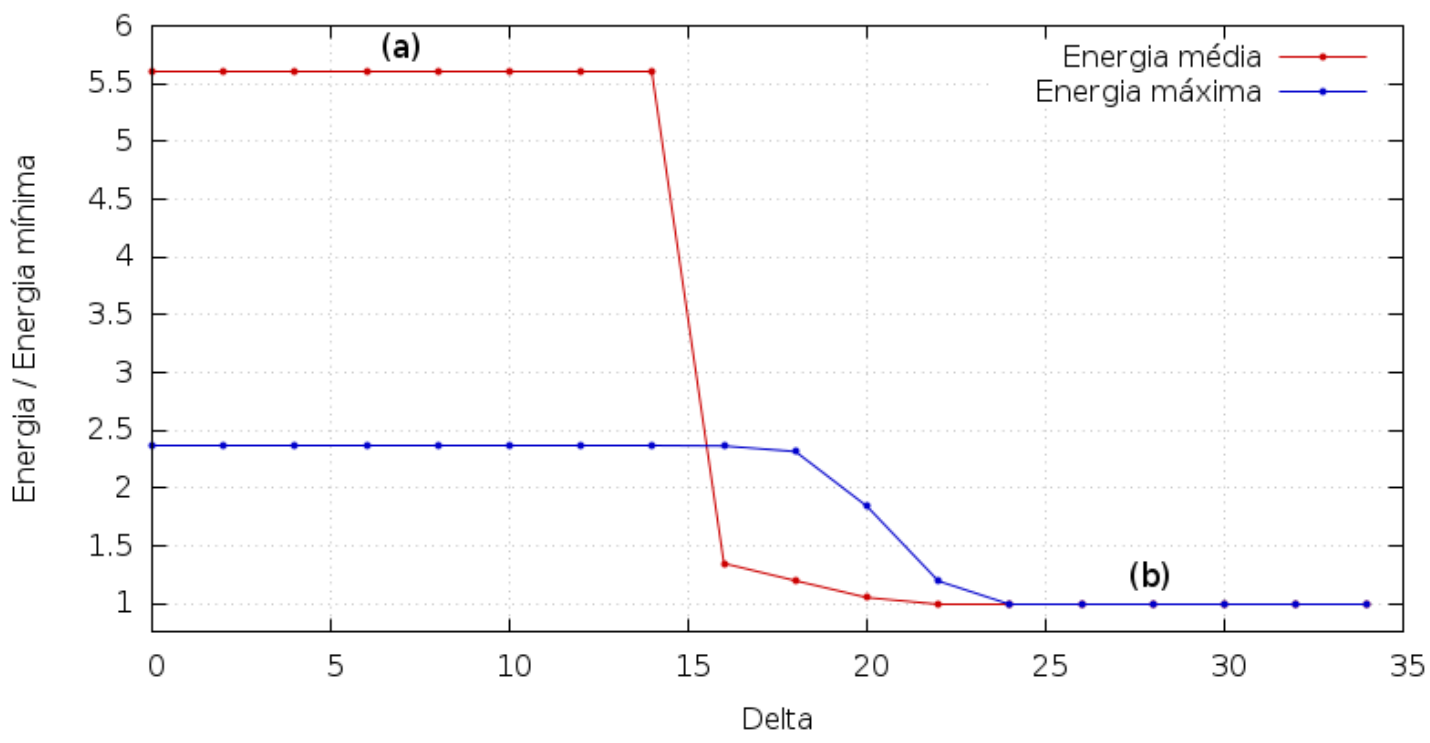

Gráaico de energias por $\Delta$

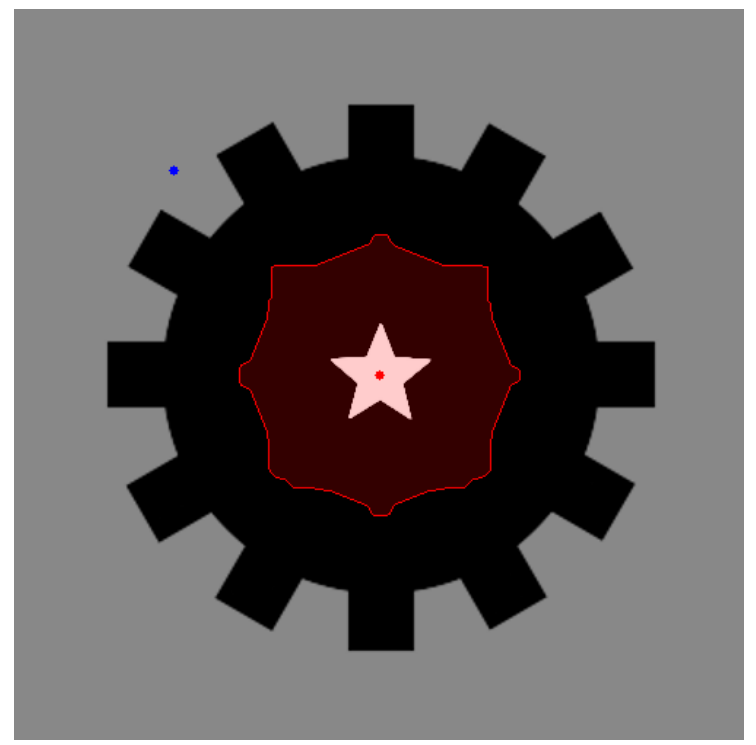

(a)

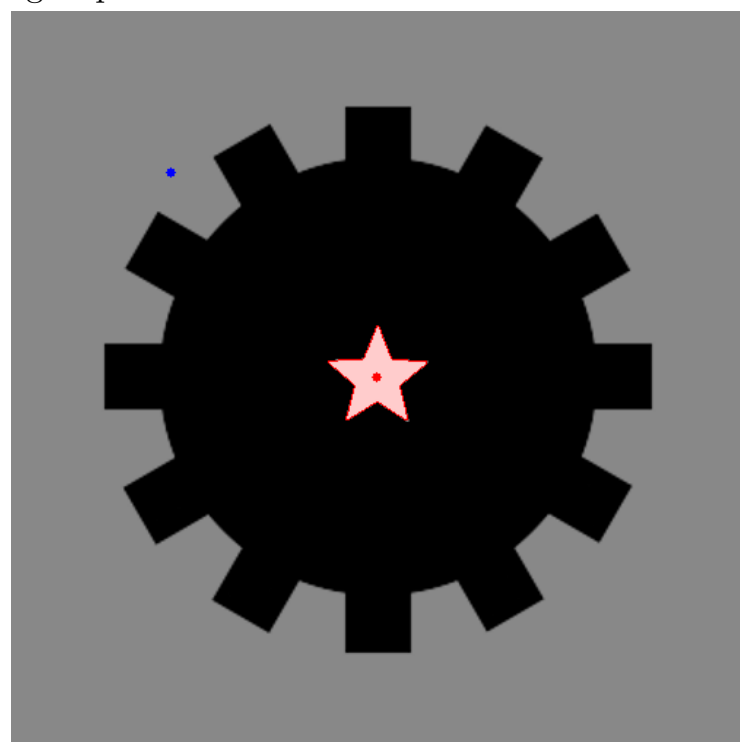

(b)

Figura 7.1: Exemplo ilustrando como a GBC-IFT não consegue segmentar a engrenagem, para nenhum valor $\Delta$.

ideia foi usar mapas de custo $C$ pré-definidos no lugar do mapa gerado pela pela primeira parte do algoritmo da RGBC-IFT, isto é, a IFT com função de custo geodésica que é calculada apenas usando as sementes de objeto.

Uma vez que obtemos mapas pré-definidos externamente, centralizamos eles no centro de massa do conjunto de sementes de objeto e executamos o algoritmo normalmente, porém estaremos direcionando o resultado da segmentação pela forma presente no mapa de custos.

A Figura 7.3 traz o exemplo de dois modelos pré-definidos de mapas de custo que utilizamos para os experimentos. Em 7.3(a), temos um modelo composto por várias formas circulares concêntricas, enquanto em 7.3(b) representa formas quadradas.

Observe que no caso do uso de um modelo de forma pré-definido, não é calculada a floresta de espalhamento $P$. No entanto, para a correta execução da RGBC-IFT basta mostrar a existência de uma floresta $P^{\prime}$, enraizada nas sementes internas, de modo que os custos do modelo escolhido C sejam não decrescentes ao longo dos caminhos de $P^{\prime}$.

Para a criação de um modelo, é necessário que as variações de escala em relação a um ponto de referência fixo da forma, mantenham as formas de escala menor inteiramente contidas dentro das formas de escalas 
Energia de segmentação

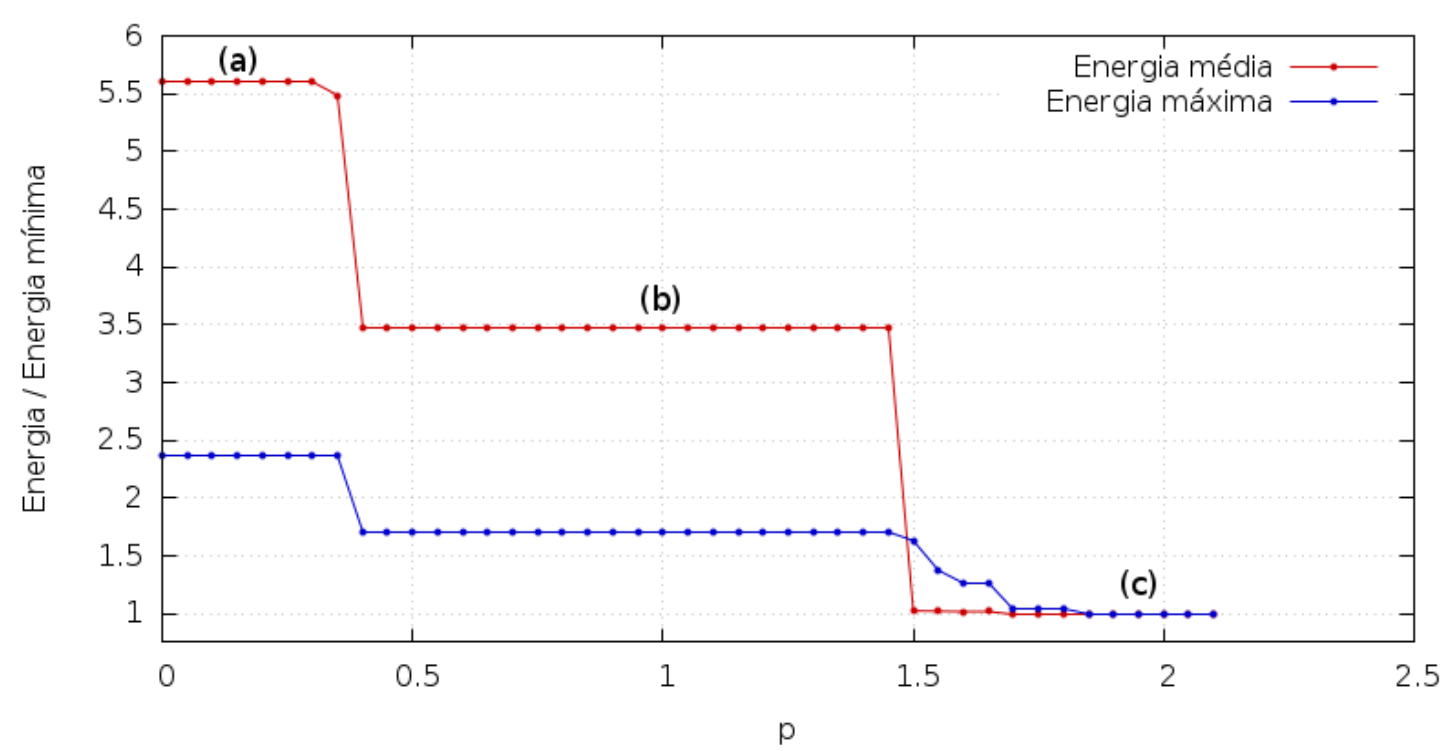

Gŕafico de energias por $p$

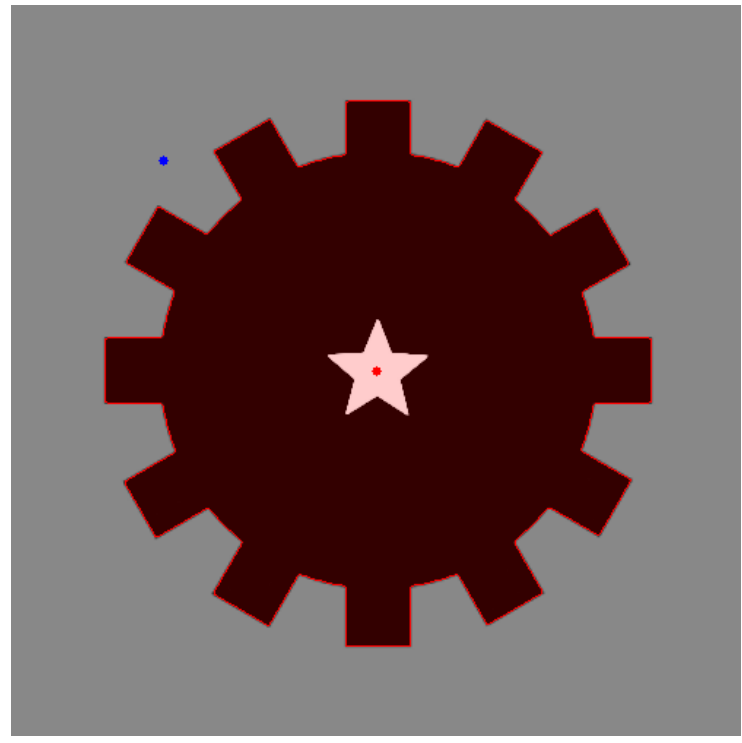

(b)

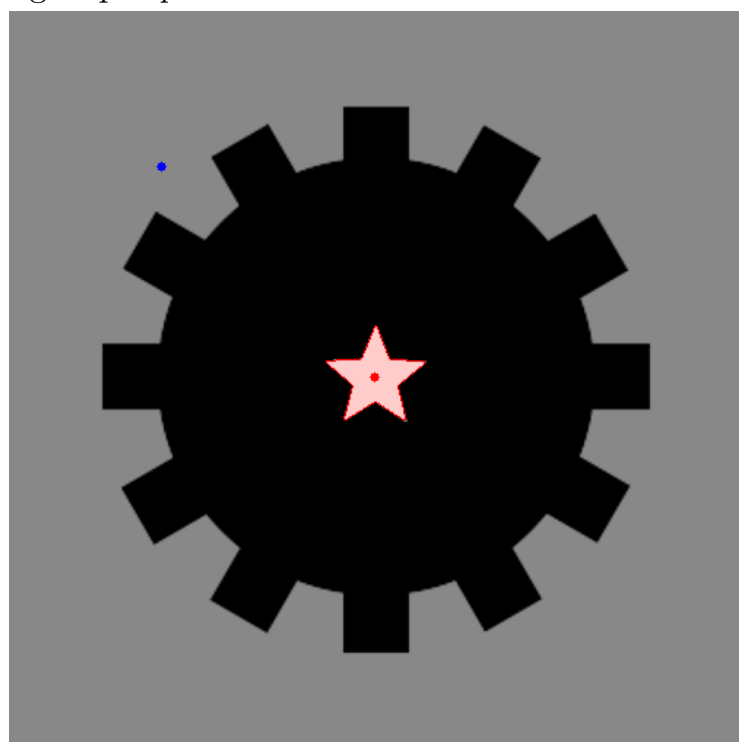

(c)

Figura 7.2: Exemplo ilustrando como a RGBC-IFT consegue segmentar a engrenagem e a estrela. A imagem relativa ao patamar (a) é igual à Figura \%.1(a).

maiores. Note que as formas convexas em estrela atendem essa restrição em relação aos seus centros de estrela, como vemos na Figura 7.4. 


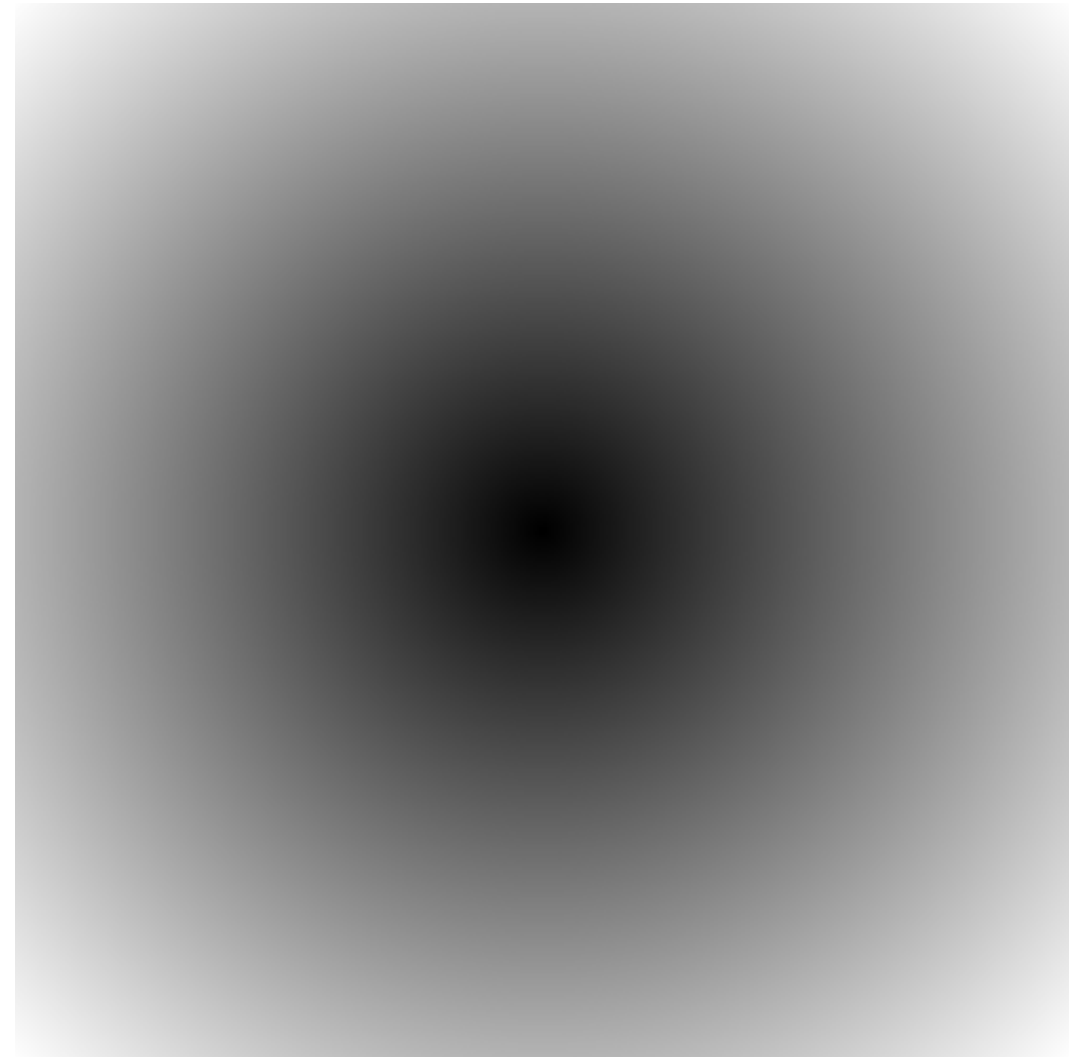

(a) Modelo de forma circular

(b) Modelo de forma quadrada

Figura 7.3: Exemplos de modelos de forma pré-definidos que podem ser usados como mapa de custos $C$. 


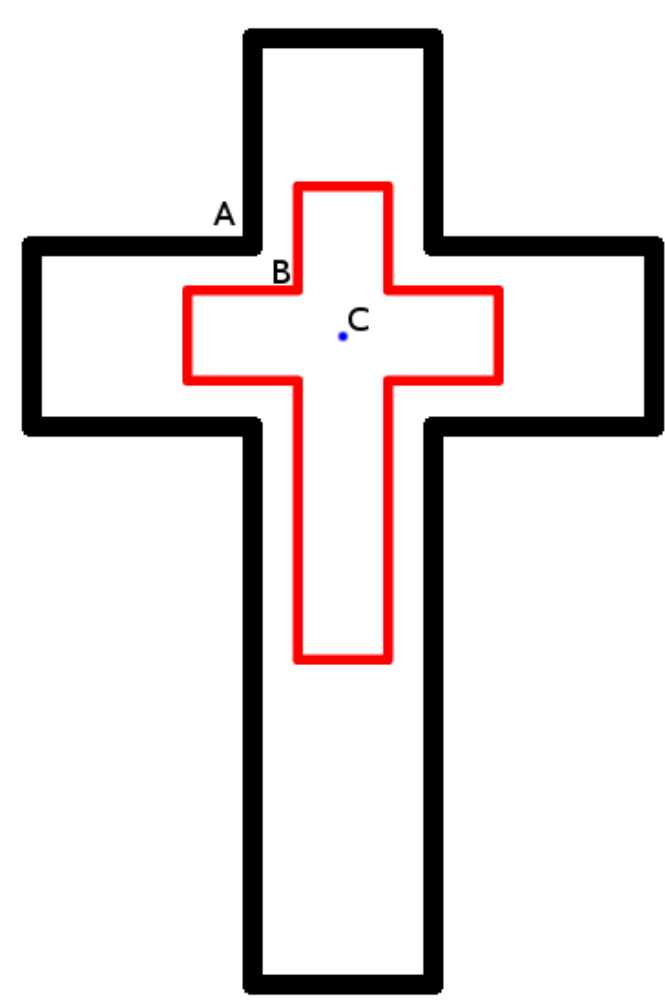

(a) Forma que pode ser usada como modelo.

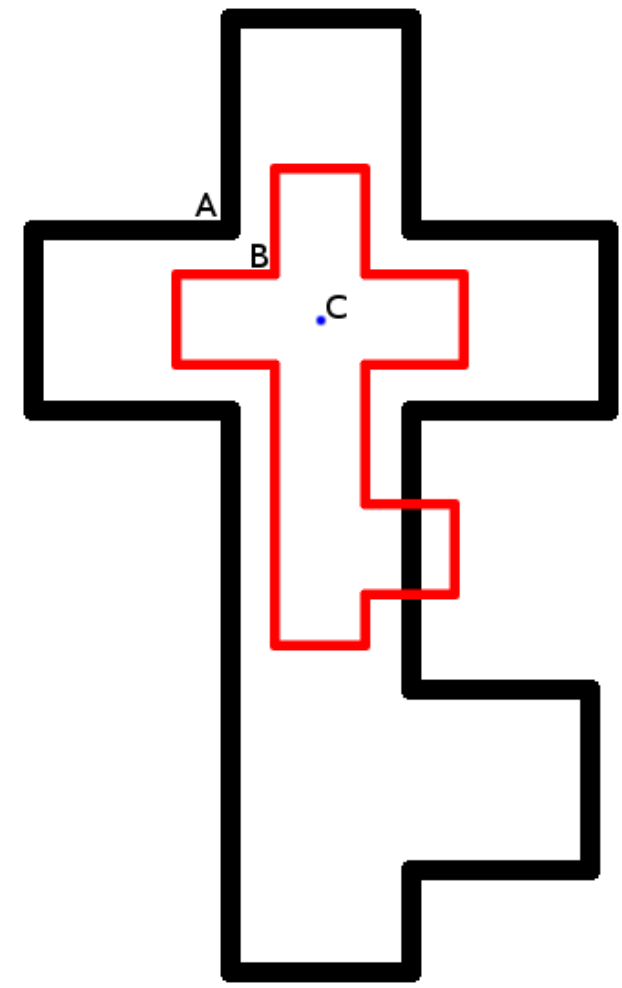

(b) Forma que não pode ser usada como modelo.

Figura 7.4: Em (a) vemos uma forma que é convexa em estrela, na qual é possivel gerar um mapa de custos pré definido de modo que as formas menores estejam sempre dentro das formas maiores. Já em (b) isso não é possível, devido à não convexidade em estrela da forma retratada em relação ao centro $C$.

\subsubsection{Experimentos}

Exibimos aqui alguns exemplos de segmentação usando os modelos de forma pré estabelecidos, comparando com alguns métodos que não fazem uso dos modelos. 


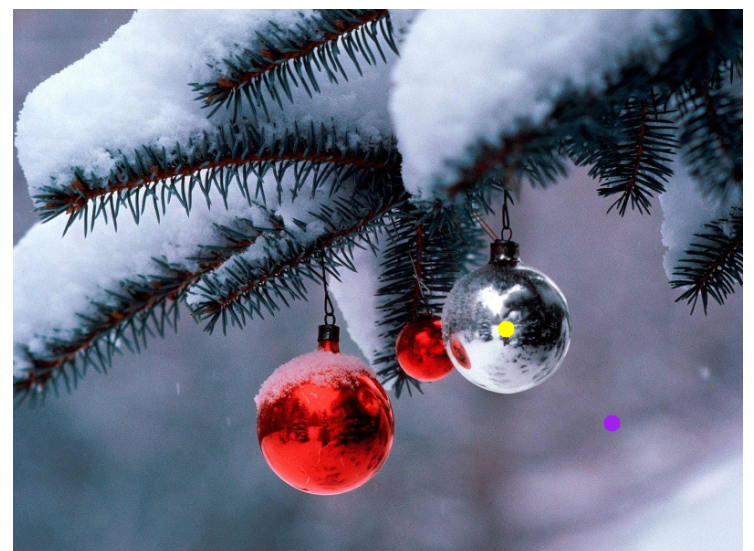

Imagem original com sementes

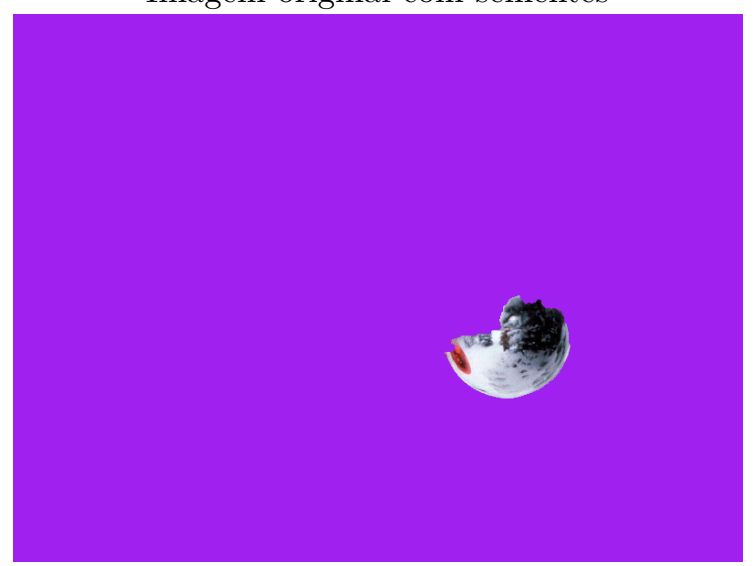

GSC-IFT

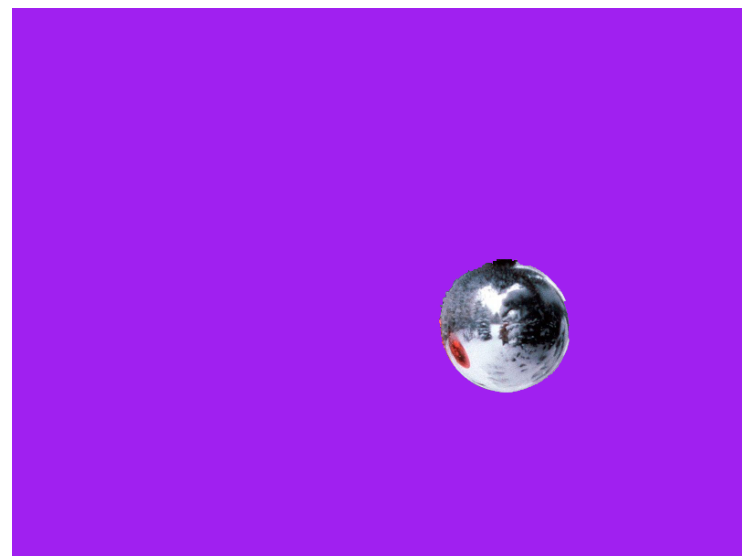

$\operatorname{RGBC}-\operatorname{IFT}(p=0.10)$

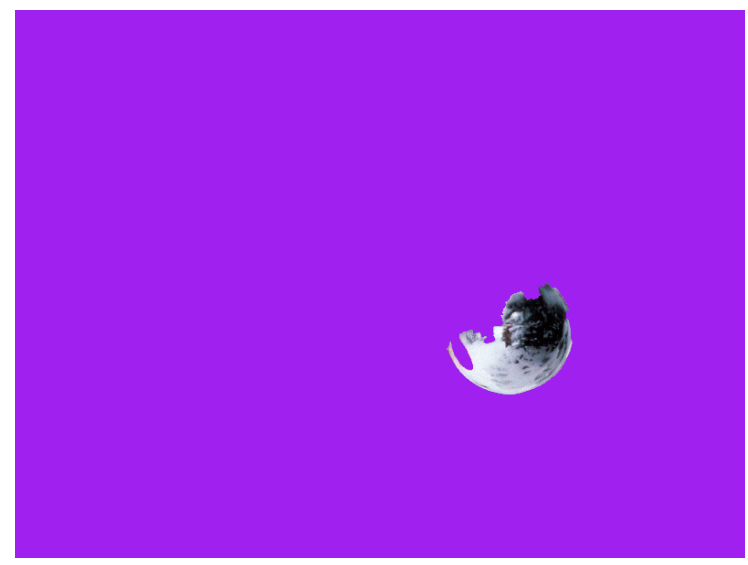

IFT

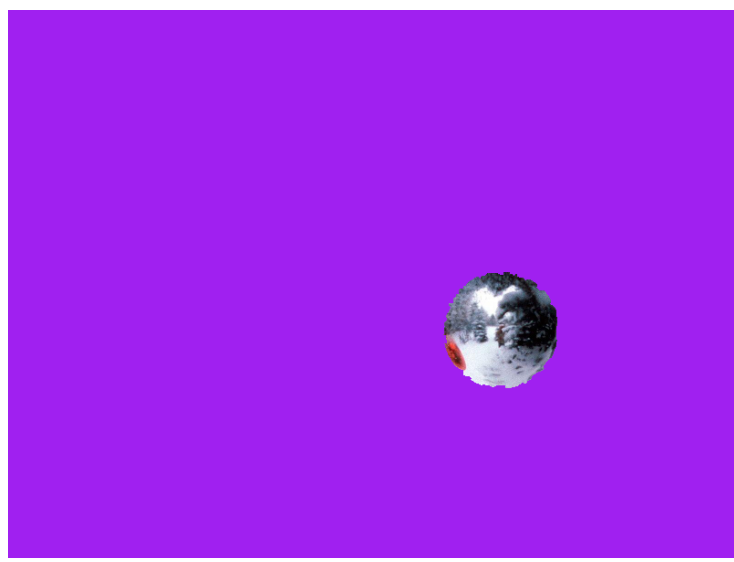

RGBC-IFT $(p=0.05)$

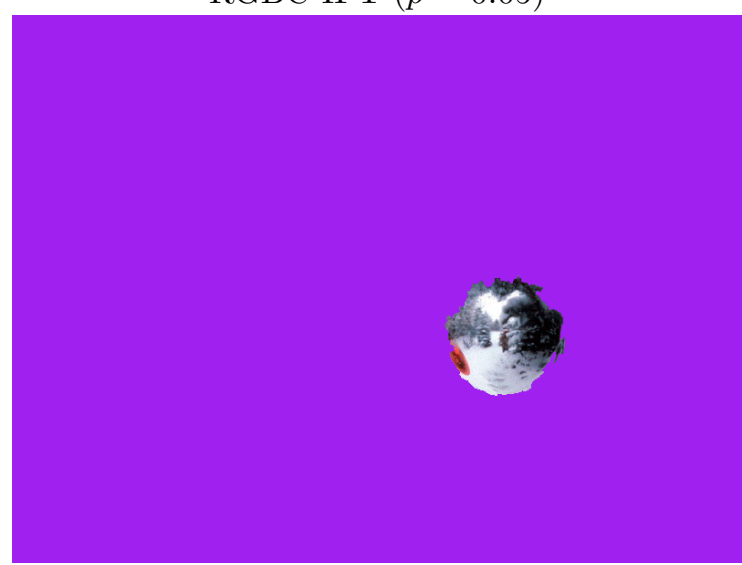

RGBC-IFT $(p=0.20)$

Figura 7.5: Exemplo de segmentação de um enfeite de natal com modelo pré-definido circular. 


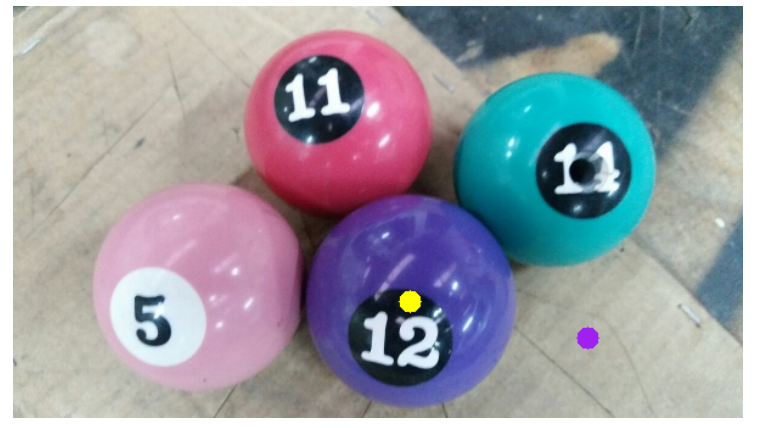

Imagem original com sementes

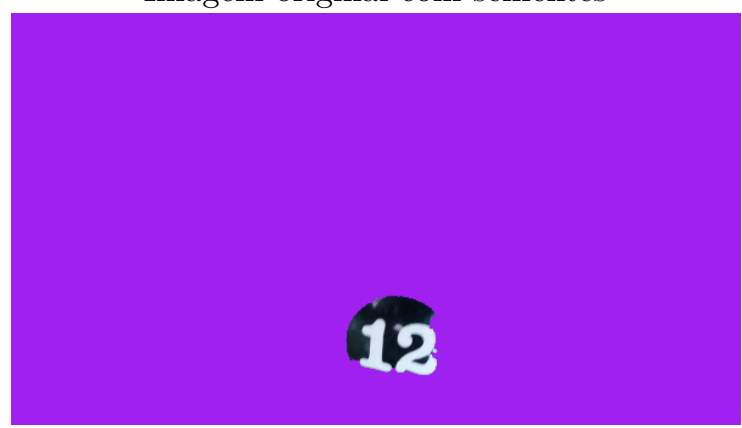

GSC-IFT

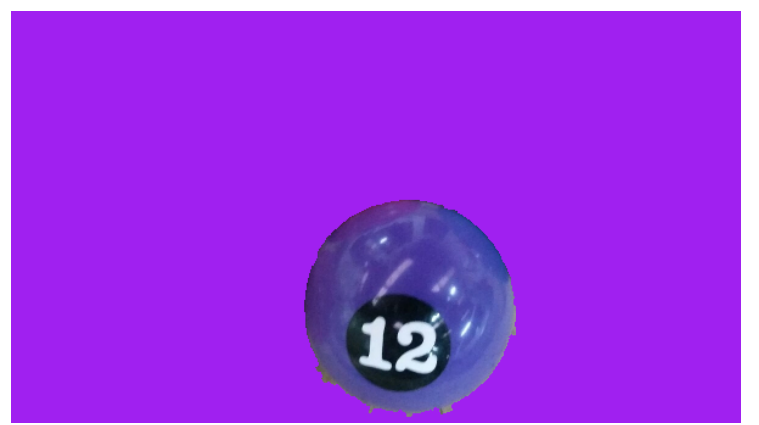

RGBC-IFT $(p=0.10)$

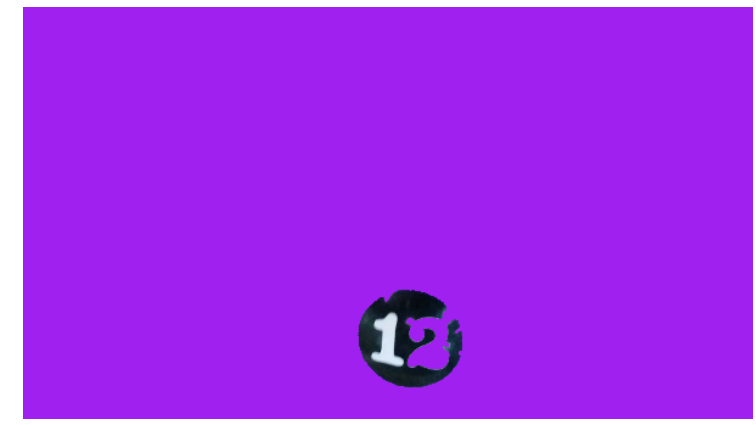

IFT

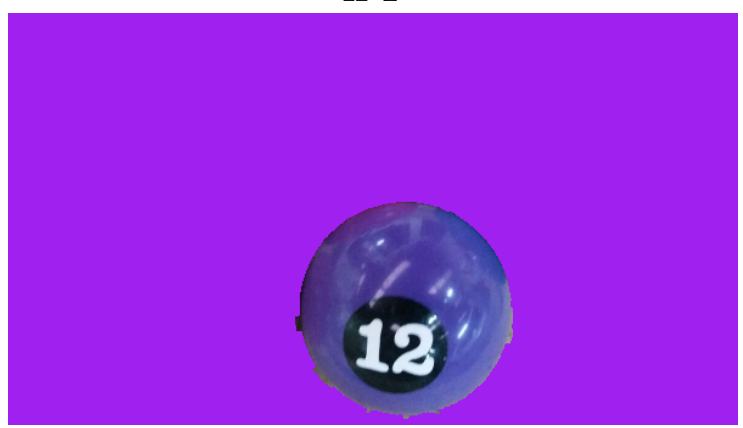

RGBC-IFT $(p=0.05)$

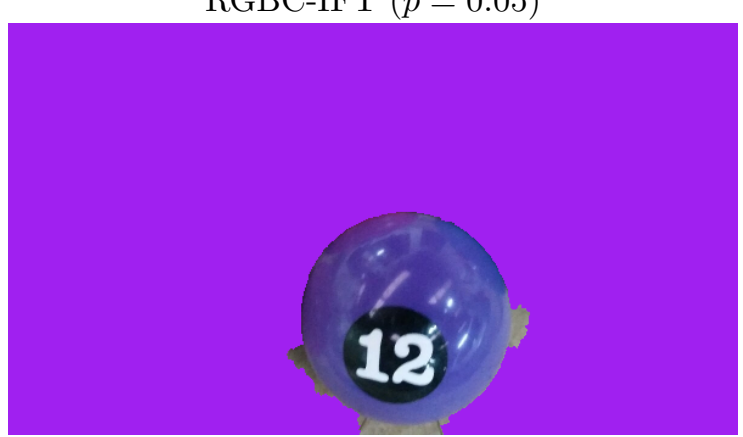

RGBC-IFT $(p=0.20)$

Figura 7.6: Exemplo de segmentação de uma bola de sinuca com modelo pré-definido circular. 


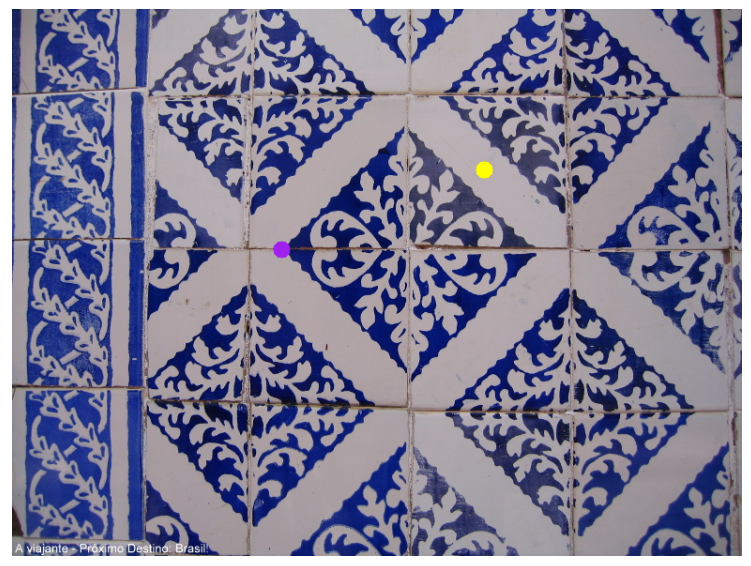

Imagem original com sementes

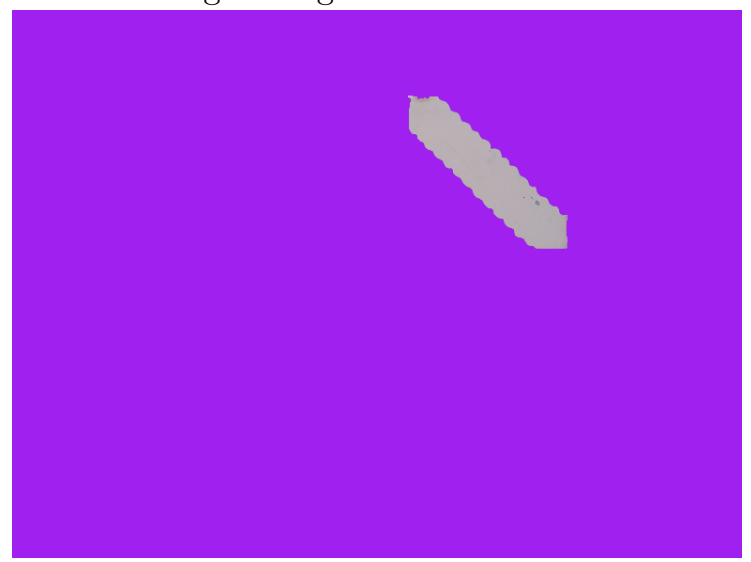

GSC-IFT

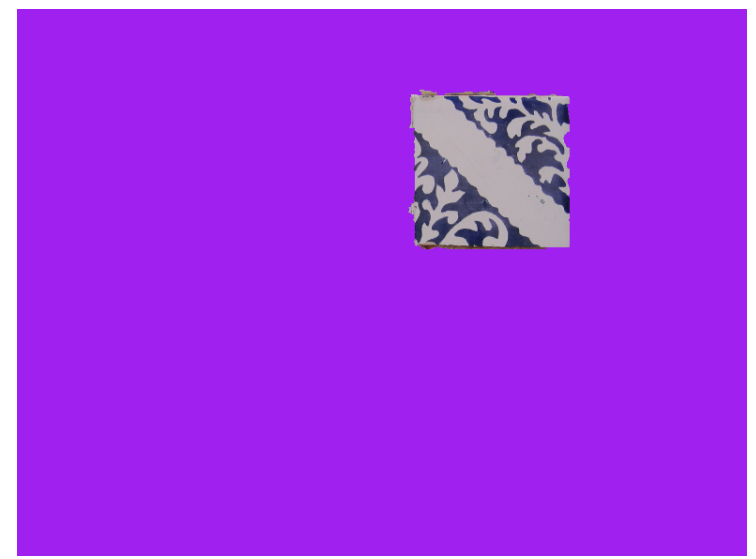

RGBC-IFT $(p=0.10)$

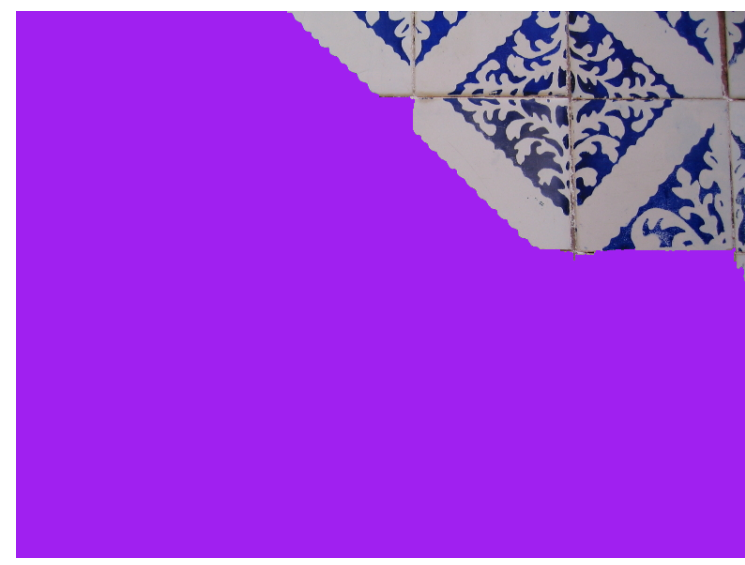

IFT

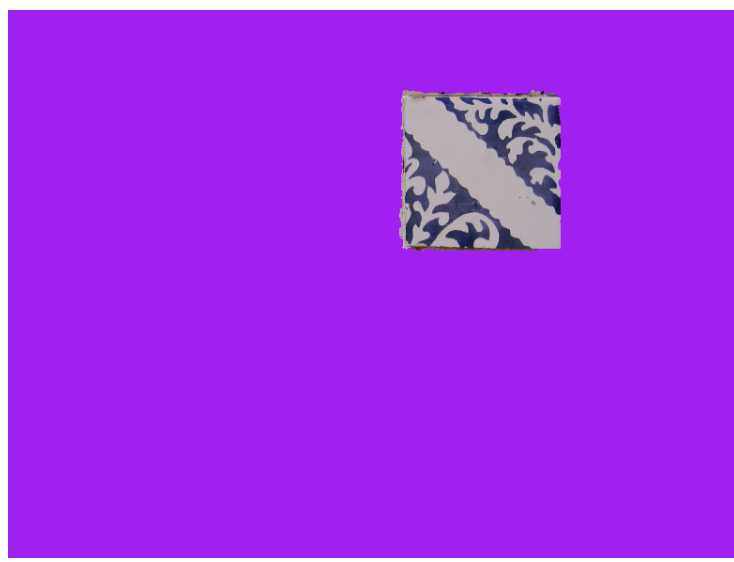

RGBC-IFT $(p=0.05)$

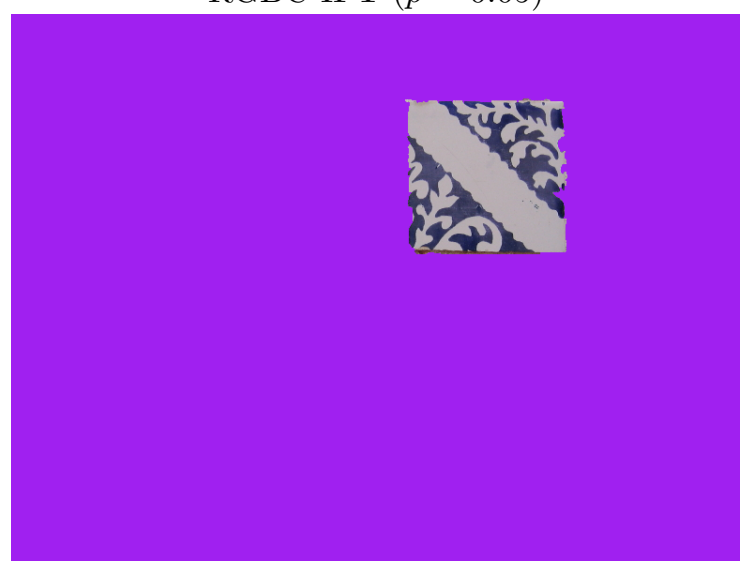

RGBC-IFT $(p=0.20)$

Figura 7.7: Exemplo de segmentação de um azulejo com modelo pré-definido quadrado. 


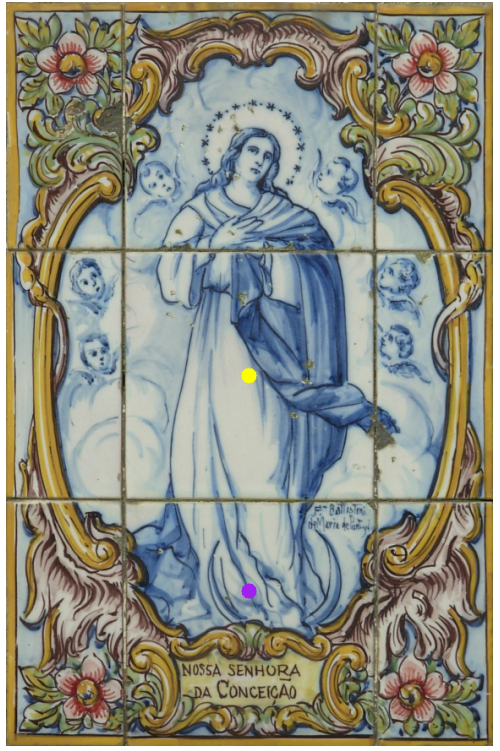

Imagem original com sementes

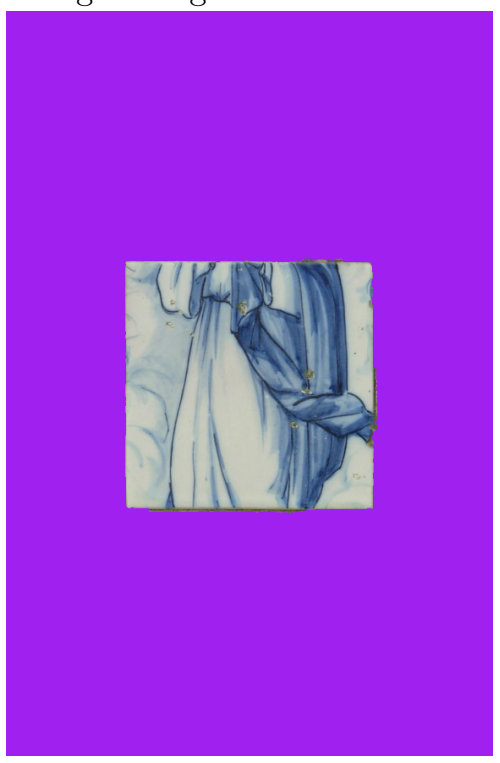

RGBC-IFT $(p=0.05)$

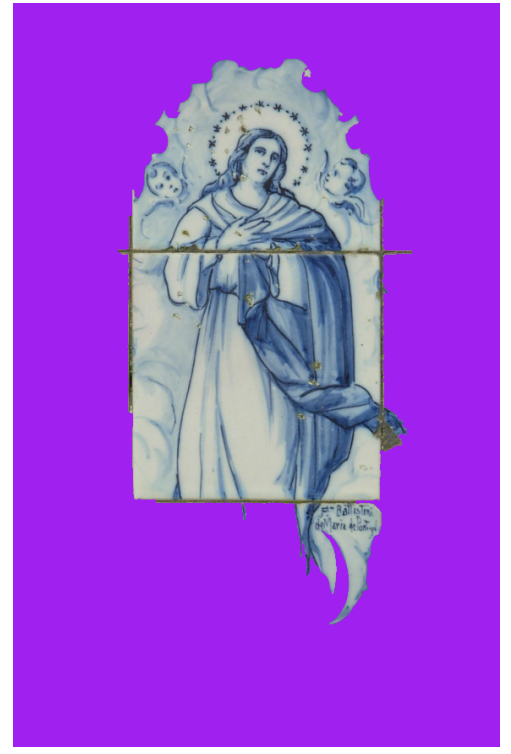

IFT

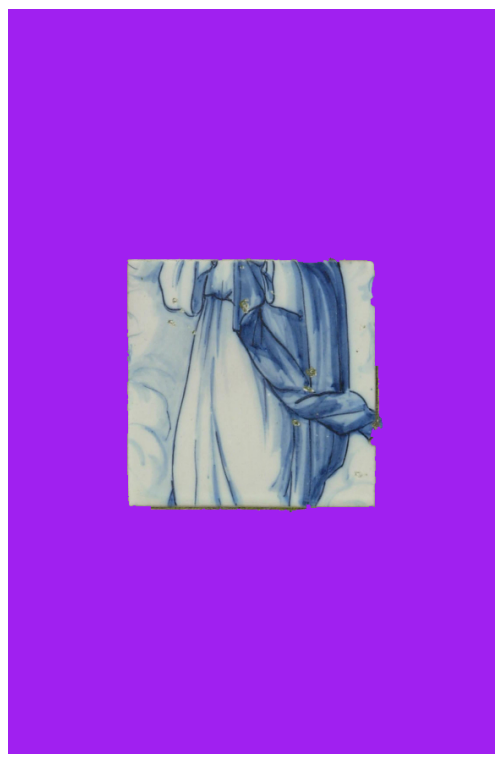

RGBC-IFT $(p=0.10)$

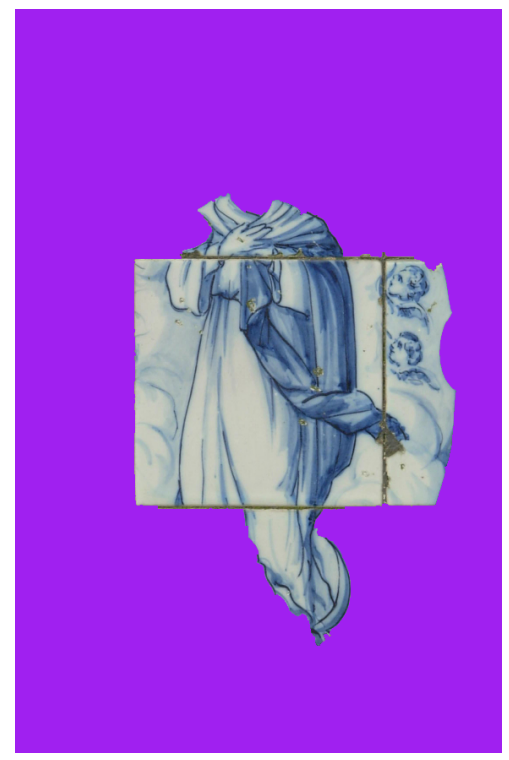

GSC-IFT

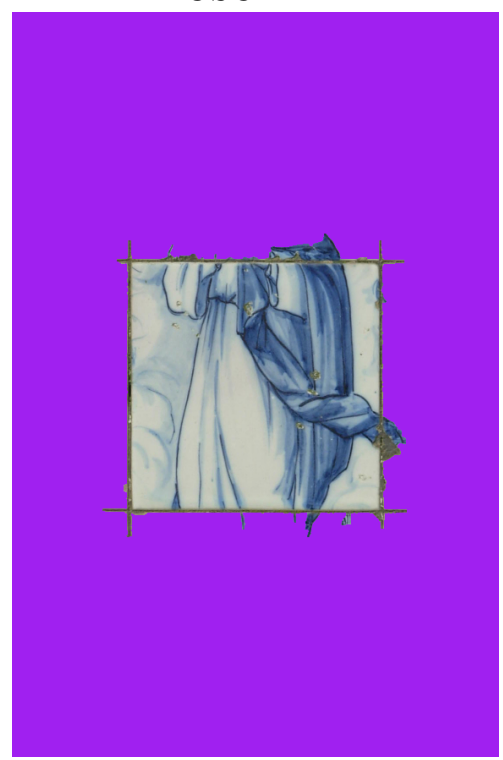

RGBC-IFT $(p=0.20)$

Figura 7.8: Exemplo de segmentação de um azulejo com modelo pré-definido quadrado. 


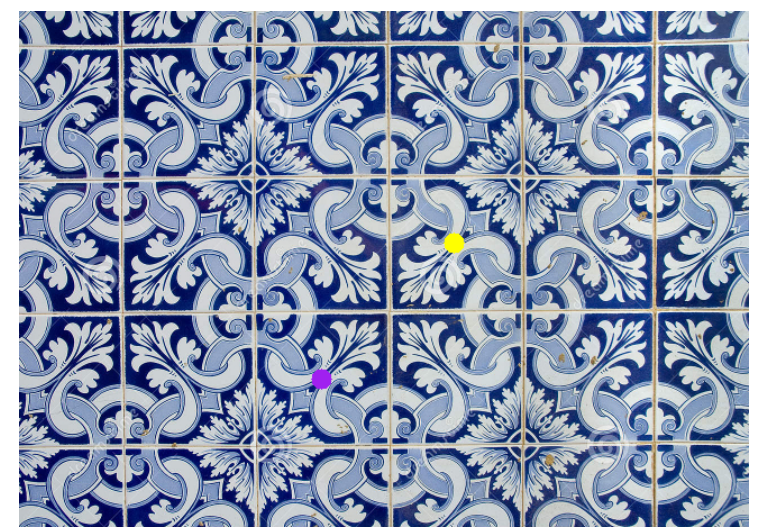

Imagem original com sementes

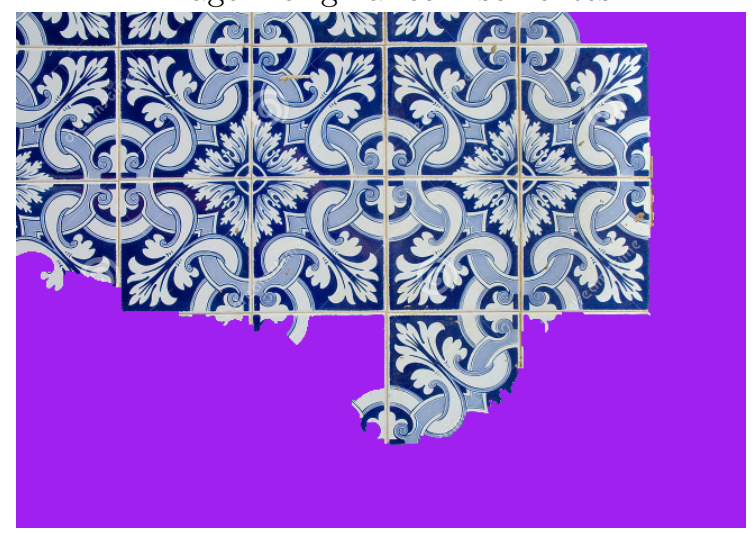

GSC-IFT

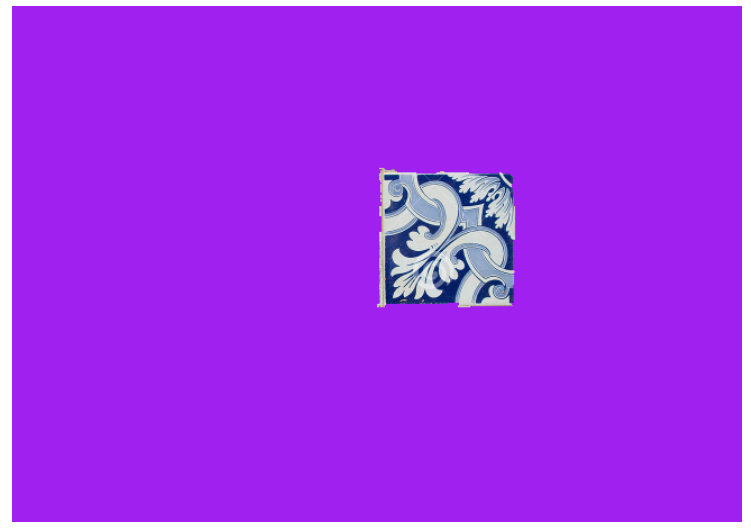

RGBC-IFT $(p=0.10)$

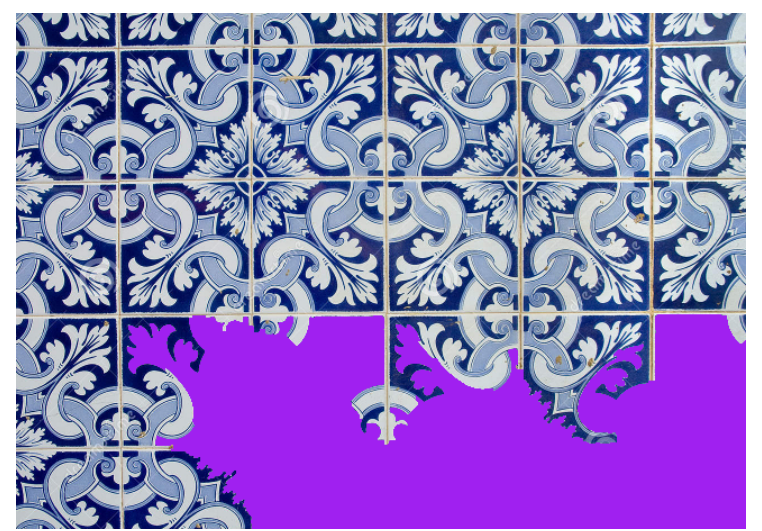

IFT

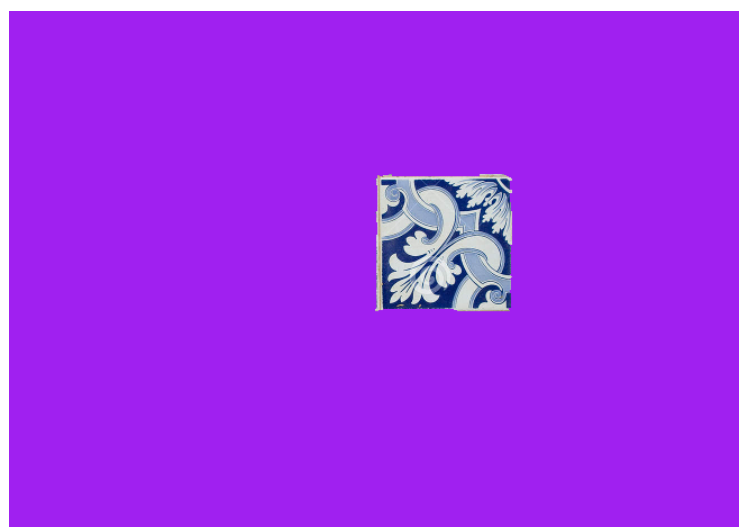

RGBC-IFT $(p=0.05)$

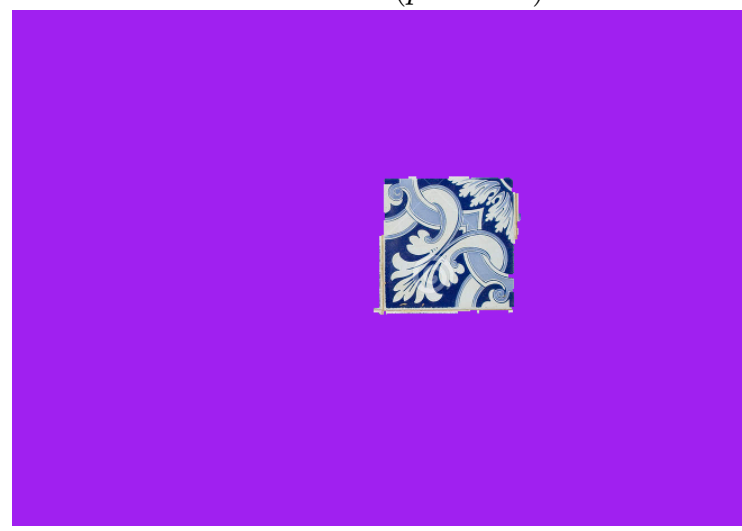

RGBC-IFT $(p=0.20)$

Figura 7.9: Exemplo de segmentação de um azulejo com modelo pré-definido quadrado. 


\section{Capítulo 8}

\section{Conclusão e trabalhos futuros}

\subsection{Considerações Finais}

A nova restrição de forma, a faixa de restrição geodésica, foi incorporada com sucesso no framework da $\varepsilon_{\infty}$-minimização por meio do desenvolvimento de um novo algoritmos que é baseado na IFT tradicional, porém com suas próprias peculiaridades, entre elas um parâmetro único para o controle da restrição que pode ser definido de forma absoluta $\Delta$ ou relativa $p$. Quando usamos o valor relativo, conseguimos contornar algumas dificuldades que surgem quando é usado um valor absoluto, conforme foi explicado na Figura 7.1. Uma limitação do método é a sensibilidade à posição das sementes internas, especialmente quando usamos os mapas de custo de modelos pré-definidos, pois neste caso, as sementes foram utilizadas para posicionar o modelo de custo na imagem.

\subsection{Contribuições}

A elaboração, com prova de corretude dos dois novos algoritmos (GBC-IFT e RGBC-IFT), com uma publicação resultante na 2014 IEEE International Conference on Image Processing [BM14].

\subsection{Trabalhos Futuros}

Um primeiro possível trabalho futuro consiste no estudo da incorporação de uma noção de localidade nas restrições propostas. Atualmente, quando há uma violação, a correção é feita em toda a extensão da borda, no entanto, podemos tentar limitar estas alterações a uma região mais próxima ao local borda onde houve esta violação.

Os algoritmos propostos podem ser incluídos em um framework de segmentação automática, nos casos onde as sementes estão disponíveis de uma fonte externa ou quando é possível gerá-las de forma automática. Estes algoritmos também são compatíveis e extensíveis para imagens 3D, então é possível implementá-los para esta finalidade.

Já com nosso modelo de formas pré-definido, é possível analisar formas pré-definidas mais complexas. Também podemos comparar esta técnica com outras que propõem uma ideia análoga de segmentar objetos de uma forma definida a priori. Os artigos [FZ05] e [VM08] nos mostram técnicas que realizam a segmentação baseada em formas pré-definidas, porém com ideias e implementações distintas. Em [FZ05], por exemplo, é usada uma pirâmide gaussiana para o tratamento de escala, enquanto nosso método com o mapa de custos pré-definido, trata o problema da escala automaticamente sem custo computacional adicional, no entanto ainda não analisamos a acurácia de cada um deles para uma dada aplicação alvo. 


\section{Referências Bibliográficas}

$\left[\mathrm{AAC}^{+} 07\right]$ C. Allène, J.Y. Audibert, M. Couprie, J. Cousty e R. Keriven. Some links between min-cuts, optimal spanning forests and watersheds. Em Proc. of the 8th Int. Symposium on Mathematical Morphology, páginas 253-264, 2007. 1

[AL07] R. Audigier e R.A. Lotufo. Watershed by image foresting transform, tie-zone, and theoretical relationship with other watershed definitions. Em Proc. of the 8th Intl. Symposium on Mathematical Morphology and its Applications to Signal and Image Processing, páginas 277-288, Oct 2007. 1

[AMO93] R.K. Ahuja, T.L. Magnanti e J.B. Orlin. Network Flows: Theory, Algorithms and Applications. Prentice-Hall, 1993. 8

[BJ01] Y.Y. Boykov e M.-P. Jolly. Interactive graph cuts for optimal boundary \& region segmentation of objects in N-D images. Em International Conference on Computer Vision (ICCV), volume 1, páginas 105-112, 2001. 1

[BK04] Y. Boykov e V. Kolmogorov. An experimental comparison of min-cut/max-flow algorithms for energy minimization in vision. IEEE Transactions on Pattern Analysis and Machine Intelligence, 26(9):1124-1137, Sep 2004. 1, 11

[BM14] C. Braz e P.A.V. Miranda. Image segmentation by image foresting transform with geodesic band constraints. Em Proceedings of the IEEE International Conference on Image Processing (ICIP), páginas 4333-4337, Oct 2014. 41

[BS07] X. Bai e G. Sapiro. Distance cut: Interactive segmentation and matting of images and videos. Em Proc. of the IEEE Intl. Conf. on Image Processing, volume 2, páginas II - 249-II - 252, 2007. 1

[CBNC10] J. Cousty, G. Bertrand, L. Najman e M. Couprie. Watershed cuts: Thinnings, shortest path forests, and topological watersheds. Trans. on Pattern Analysis and Machine Intelligence, 32:925-939, 2010. 1

[CG12] K. S. Camilus e V. K. Govindan. A review on graph based segmentation. Intl. Journal of Image, Graphics and Signal Processing, 4(5):1-13, 2012. 1

[CLLH10] C.Couprie, L.Grady, L.Najman e H.Talbot. Power watersheds: A unifying graph-based optimization framework. Trans. on Pattern Anal. and Machine Intelligence, 99, 2010. 1, 11, 12

[CUFM12a] K.C. Ciesielski, J.K. Udupa, A.X. Falcão e P.A.V. Miranda. Fuzzy connectedness image segmentation in graph cut formulation: A linear-time algorithm and a comparative analysis. Journal of Mathematical Imaging and Vision, 2012. 1, 11, 12

[CUFM12b] K.C. Ciesielski, J.K. Udupa, A.X. Falcão e P.A.V. Miranda. A unifying graph-cut image segmentation framework: algorithms it encompasses and equivalences among them. Em Proc. of SPIE on Medical Imaging: Image Processing, volume 8314, 2012. 1, 11

[CUSZ07] K.C. Ciesielski, J.K. Udupa, P.K. Saha e Y. Zhuge. Iterative relative fuzzy connectedness for multiple objects with multiple seeds. Computer Vision and Image Understanding, 107(3):160182, 2007. 1

[Dij59] E.W. Dijkstra. A note on two problems in connexion with graphs. Numerische Mathematik, 1:269-271, 1959. 8 
[FB04] A.X. Falcão e F.P.G. Bergo. Interactive volume segmentation with differential image foresting transforms. IEEE Trans. on Medical Imaging, 23(9):1100-1108, 2004. 1, 5

[FdC01] A.X. Falcão e B.S. da Cunha. Multiscale Shape Representation by Image Foresting Transform. Em Milan Sonka e Kenneth Hanson, editors, Proceedings of SPIE on Medical Imaging, volume 4322, páginas 1091-1100, San Diego, CA, Feb 2001. 5

[FdCL01] A.X. Falcão, B.S. da Cunha e R.A. Lotufo. Design of connected operators using the image foresting transform. Em Proceedings of SPIE on Medical Imaging, volume 4322, páginas 468479, Feb 2001. 5

[FSL04] A.X. Falcão, J. Stolfi e R.A. Lotufo. The image foresting transform: Theory, algorithms, and applications. IEEE Transactions on Pattern Analysis and Machine Intelligence, 26(1):19-29, 2004. 1, 2, 7, 9

[FZ05] D. Freedman e Tao Zhang. Interactive graph cut based segmentation with shape priors. Em Computer Vision and Pattern Recognition, 2005. CVPR 2005. IEEE Computer Society Conference on, volume 1, páginas 755-762 vol. 1, June 2005. 41

[Gra06] L. Grady. Random walks for image segmentation. IEEE Trans. Pattern Anaysis and Machine Intelligence, 28(11):1768-1783, 2006. 1

$\left[\mathrm{GRC}^{+} 10\right]$ V. Gulshan, C. Rother, A. Criminisi, A. Blake e A. Zisserman. Geodesic star convexity for interactive image segmentation. Em Proc. of Computer Vision and Pattern Recognition, páginas 3129-3136, 2010. 2, 13, 21

[GW92] R.C. Gonzalez e R.E. Woods. Digital Image Processing. Addison-Wesley, Reading, MA, USA, 1992. 1

[LG12] O. Lézoray e L. Grady. Image Processing and Analysis with Graphs: Theory and Practice. CRC Press, California, USA, 2012. 1, 2

[Man14] L.A.C. Mansilla. Transformada imagem-floresta com funções de conexidade não suaves: Pesos adaptativos, polaridade de borda e restrições de forma. Dissertação de Mestrado, Universidade de São Paulo, Instituto de Matemática e Estatística, Fev 2014. 13

[MF09] P.A.V. Miranda e A.X. Falcão. Links between image segmentation based on optimum-path forest and minimum cut in graph. Journal of Mathematical Imaging and Vision, 35(2):128142, 2009. 1, 7, 9

[MFS11] P.A.V. Miranda, A.X. Falcão e T.V. Spina. The riverbed approach for user-steered image segmentation. Em Proc. of the International Conference on Image Processing (ICIP), Brussels, Belgium, 2011. IEEE. to appear. 5

[MJM13] L.A.C. Mansilla, M.P. Jackowski e P.A.V. Miranda. Image foresting transform with geodesic star convexity for interactive image segmentation. Em Proceedings of the IEEE International Conference on Image Processing (ICIP), páginas 4054-4058, Set 2013. 2, 21

[MM13a] L.A.C. Mansilla e P.A.V. Miranda. Image segmentation by oriented image foresting transform: Handling ties and colored images. Em 18th Intl. Conf. on Digital Signal Processing, páginas 1-6, Greece, Jul 2013. 17, 19

[MM13b] L.A.C. Mansilla e P.A.V. Miranda. Image segmentation by oriented image foresting transform with geodesic star convexity. Em 15th International Conference on Computer Analysis of Images and Patterns (CAIP), volume 8047, páginas 572-579, 2013. 2, 28

[MM14] P.A.V. Miranda e L.A.C. Mansilla. Oriented image foresting transform segmentation by seed competition. IEEE Transactions on Image Processing, 23(1):389-398, 2014. 2, 17, 19

[OS01] S.D. Olabarriaga e A.W.M. Smeulders. Interaction in the segmentation of medical images: A survey. Medical Image Analysis, 5(2):127-142, 2001. 1

[SL07] A.K. Sinop e L.Grady. A seeded image segmentation framework unifying graph cuts and random walker which yields a new algorithm. Em Proc. of the 11th International Conference on Computer Vision (ICCV), páginas 1-8. IEEE, 2007. 1 
[VK05] V. Vezhnevets e V. Konouchine. "growcut- interactive multi-label N-D image segmentation by cellular automata. Em Proc. Graphicon., páginas 150-156, 2005. 1

[VM08] N. Vu e B.S. Manjunath. Shape prior segmentation of multiple objects with graph cuts. Em Computer Vision and Pattern Recognition, 2008. CVPR 2008. IEEE Conference on, páginas 1-8, June 2008. 41

[WC05] J. Wang e M.F. Cohen. An iterative optimization approach for unified image segmentation and matting. Em Proceedings of the Tenth International Conference on Computer Vision (ICCV), páginas 936-943, Washington, DC, USA, 2005. IEEE Computer Society. 1 